\title{
Topological full groups of ample groupoids with applications to graph algebras
}

\author{
Petter Nyland* and Eduard Ortega ${ }^{\dagger}$ \\ Department of Mathematical Sciences \\ Faculty of Information Technology and Electrical Engineering \\ NTNU - Norwegian University of Science and Technology \\ Trondheim, Norway \\ *petter.nyland@ntnu.no \\ †eduard.ortega@ntnu.no
}

Received 27 September 2018

Accepted 3 January 2019

Published 18 April 2019

\begin{abstract}
We study the topological full group of ample groupoids over locally compact spaces. We extend Matui's definition of the topological full group from the compact to the locally compact case. We provide two general classes of étale groupoids for which the topological full group, as an abstract group, is a complete isomorphism invariant, hereby extending Matui's Isomorphism Theorem. As an application, we study graph groupoids and their topological full groups, and obtain sharper results for this class. The machinery developed in this process is used to prove an embedding theorem for ample groupoids, akin to Kirchberg's Embedding Theorem for $C^{*}$-algebras. Consequences for graph $C^{*}$-algebras and Leavitt path algebras are also spelled out. In particular, we improve on a recent embedding theorem of Brownlowe and Sørensen for Leavitt path algebras.
\end{abstract}

Keywords: Topological full group; ample groupoid; graph groupoid; AF-groupoid; graph $C^{*}$-algebra; Leavitt path algebra.

Mathematics Subject Classification 2010: 22A22, 37B05, 54H20, 37B10, 46L05, 16 S10

\section{Introduction}

\section{Background}

The study of (topological) full groups in the setting of topological dynamics was initiated by Giordano, Putnam and Skau [27]. This was inspired by the work of Dye 23] in the measurable setting, and by Krieger's study of so-called ample groups

\footnotetext{
${ }^{*}$ Corresponding author.
}

This is an Open Access article published by World Scientific Publishing Company. It is distributed under the terms of the Creative Commons Attribution 4.0 (CC-BY) License. Further distribution of this work is permitted, provided the original work is properly cited. 
on the Cantor space [32]. For Cantor minimal systems, Giordano, Putnam and Skau showed that certain distinguished subgroups of the full group determine completely the orbit equivalence class, the strong orbit equivalence class, and the flip conjugacy class, respectively, of the system. The full group of a Cantor system (i.e. a $\mathbb{Z}$-action on a Cantor space) consists of all homeomorphisms of the Cantor space which leave the orbits invariant. Roughly speaking, the topological full group is the subgroup of the full group consisting of those homeomorphisms which additionally preserve the orbits in a continuous manner. Giordano, Putnam and Skau also connected the dynamics with the theory of $C^{*}$-algebras, via the crossed product construction and its $K$-theory [26]. Thus, they exhibited a strong relationship between these, a priori, quite different mathematical structures.

This is but one example of the rich interplay between dynamical systems and $C^{*}$-algebras. (This interplay essentially goes all the way back to the inception of the field by Murray and von Neumann [53.) Another prominent example of this interplay is the connection between shifts of finite type and Cuntz-Krieger algebras; discovered by Cuntz and Krieger in the early eighties [17. In the setting of irreducible one-sided shifts of finite type, Matsumoto defined the topological full group of such a dynamical system and proved that this group determines the shift up to continuous orbit equivalence, and also the associated Cuntz-Krieger algebra up to diagonal preserving isomorphism [42, 43]. This paralleled Giordano, Putnam and Skau's results, although the dynamical systems were quite different. For instance, the former has no periodic points whereas the latter has a dense set of periodic points.

Using topological groupoids to model dynamical systems has unified many of these seemingly different connections between dynamics and $C^{*}$-algebras. Whenever one has a dynamical system of some sort, one may typically associate to it a topological groupoid, and from the groupoid one can construct its groupoid $C^{*}$-algebra. In many cases, isomorphism of such groupoids correspond to some suitable notion of continuous orbit equivalence of the dynamical systems, and also to diagonal preserving isomorphism of the groupoid $C^{*}$-algebras [10, 40, 41] 44]. That groupoid isomorphism corresponds to diagonal preserving isomorphism of the $C^{*}$-algebras (in the topologically principal case) is due to the pioneering work of Renault [61]. This reconstruction result has recently been generalized in e.g. [15]; wherein it is also shown that by adding more structure on the groupoids, such as gradings, one can recover stronger types of equivalence of the dynamical systems.

In [48], Matui defined the topological full group of an étale groupoid with compact unit space. His definition generalized virtually all the previously given definitions for different kinds of dynamical systems at one fell swoop. Matui realized that homeomorphisms which preserve orbits in a continuous manner are always given by full bisections from the associated groupoid. In the subsequent paper [49] Matui proved (among other things) a remarkable isomorphism theorem. Suppressing some assumptions, this theorem says that any two minimal étale groupoids 
over a Cantor space are isomorphic, as topological groupoids, if and only if their topological full groups ${ }^{\text {a }}$ are isomorphic, as abstract groups. Matui's Isomorphism Theorem generalized the results of Giordano, Putnam and Skau, and Matsumoto, and others.

The study of topological full groups has also found interesting applications to group theory. Matui's isomorphism theorem means that one can classify the groupoids (and therefore any underlying dynamics, and the $C^{*}$-algebras) in terms of the topological full group. However, by going the other direction, one can use étale groupoids to distinguish certain discrete groups. Given two discrete groups, say in terms of their generators and relations, it can be hard to tell whether they are isomorphic or not. But if one can realize these groups as topological full groups (or distinguished subgroups) of some groupoids, then one can use the groupoids (i.e. the dynamics) to tell the groups apart - as one often has much dynamical information about the groupoids. For instance, this was the strategy used by Brin to show that Thompson's group $V$ is not isomorphic to its two-dimensional analog $2 V$ [6] (although he did not consider the groupoid explicitly). A more recent application of this form is by Matte Bon [45] who showed that the higher-dimensional Thompson group $^{\mathrm{b}} n V$ embeds into $m V$ if and only if $n \leq m$. Matte Bon's paper also includes a novel approach to Matui's Isomorphism Theorem in terms of a certain dichotomy for such groupoids. Another application is that topological full groups have provided new examples of groups with exotic properties. Most notably, topological full groups (or more precisely, their commutator subgroups) of Cantor minimal systems provided the first examples of finitely generated simple groups that are amenable (and infinite) [29]. On another note, topological full groups arising from non-amenable groups acting minimally and topologically free on the Cantor space were recently shown to be $C^{*}$-simple [7].

Topological full groups have also found their way into Lawson's program of non-commutative Stone duality [38]. In [39], the topological full group of an étale groupoid is shown to coincide with the group of units of the so-called Tarski monoid to which the groupoid corresponds under non-commutative Stone duality.

\section{Our results}

The main motivation for the present paper was Matsumoto and Matui's work on irreducible one-sided shifts of finite type mentioned above. If we rephrase their work in terms of (directed) graphs, then they showed that for two strongly connected finite graphs $E$ and $F$ the following are equivalent:

(1) The shifts $\left(E^{\infty}, \sigma_{E}\right)$ and $\left(F^{\infty}, \sigma_{F}\right)$ are continuously orbit equivalent.

(2) The graph groupoids $\mathcal{G}_{E}$ and $\mathcal{G}_{F}$ are isomorphic as topological groupoids.

\footnotetext{
aActually, the same is true for several distinguished subgroups of the topological full group as well, such as its commutator subgroup. See [49, 54 for details.

${ }^{\mathrm{b}} \mathrm{It}$ is known that the groups $n V$ are all non-isomorphic [4].
} 
(3) There is an isomorphism of the graph $C^{*}$-algebras $C^{*}(E)$ and $C^{*}(F)$ which maps the diagonal $\mathcal{D}(E)$ onto $\mathcal{D}(F)$.

(4) The topological full groups $\llbracket \mathcal{G}_{E} \rrbracket$ and $\llbracket \mathcal{G}_{F} \rrbracket$ are isomorphic as abstract groups.

The equivalence of (1), (2) and (3) above have since been generalized to more general graphs which need neither be finite nor strongly connected [10, 12, Our initial goal was to study the topological full group $\llbracket \mathcal{G}_{E} \rrbracket$ of general graph groupoids $\mathcal{G}_{E}$ and see if we could also add statement (4) to said equivalence.

Matui's Isomorphism Theorem [49] Theorem 3.10] gives the equivalence of (2) and (4) above for the general class of ample effective Hausdorff minimal second countable groupoids over (compact) Cantor spaces (see Sec. 2.3 for definitions). This covers in particular graph groupoids of strongly connected finite graphs. In light of this we attempted to extend Matui's Isomorphism Theorem a little further in order to cover graph groupoids of more general graphs. To do this it is necessary to relax both the compactness assumption of the unit space (which corresponds to the graph having finitely many vertices) and the minimality assumption (which corresponds to strong connectedness of the graph).

As our main findings, we first describe two modest extensions of Matui's Isomorphism Theorem that apply to general ample groupoids. Then, we describe two (sharper) isomorphism theorems for the class of graph groupoids. Finally, we present a novel embedding theorem for ample groupoids. First of all we have to extend the definition of the topological full group to the locally compact setting. This is done in Definition 3.2, where we stipulate that the homeomorphisms in the topological full group should be compactly supported (in addition to being induced by bisections). This seems a natural choice, as we then retain the "finitary" nature of the elements in the topological full group, as well as the countability of the topological full group (for second countable groupoids). Additionally, most of the arguments from 49] still work with suitable modifications. For an ample groupoid $\mathcal{G}$ we denote its unit space by $\mathcal{G}^{(0)}$. The topological full group of $\mathcal{G}$ is denoted by $\llbracket \mathcal{G} \rrbracket$. And the commutator subgroup of $\llbracket \mathcal{G} \rrbracket$ is denoted by $\mathrm{D}(\llbracket \mathcal{G} \rrbracket)$. The first of these isomorphism theorems is a straightforward extension of Matui's Isomorphism Theorem which relaxes the compactness assumption on $\mathcal{G}^{(0)}$ and the second countability assumption on $\mathcal{G}$.

Theorem A (cf. Theorem [7.2, [49, Theorem 3.10]). Suppose $\mathcal{G}_{1}$ and $\mathcal{G}_{2}$ are effective ample minimal Hausdorff groupoids whose unit spaces have no isolated points. Then following are equivalent:

(1) $\mathcal{G}_{1} \cong \mathcal{G}_{2}$ as topological groupoids.

(2) $\llbracket \mathcal{G}_{1} \rrbracket \cong \llbracket \mathcal{G}_{2} \rrbracket$ as abstract groups.

(3) $\mathrm{D}\left(\llbracket \mathcal{G}_{1} \rrbracket\right) \cong \mathrm{D}\left(\llbracket \mathcal{G}_{2} \rrbracket\right)$ as abstract groups.

We mention that when restricting to the class of graph groupoids we are also able to relax the minimality assumption in Theorem $\mathrm{A}$ substantially (see Theorem $\mathrm{C}$ below). The second isomorphism theorem replaces the minimality assumption with 
a significantly weaker "mixing property" that we call non-wandering (see Definition 7.8). However, the result does not apply to the commutator subgroups. And we also require the unit spaces to be second countable. (By a locally compact Cantor space we mean either the compact Cantor space or the locally compact non-compact Cantor space (up to homeomorphism) cf. Sec. 2.1)

Theorem B (cf. Theorem [7.10). Let $\mathcal{G}_{1}$ and $\mathcal{G}_{2}$ be effective ample Hausdorff groupoids over locally compact Cantor spaces. If, for $i=1,2, \mathcal{G}_{i}$ is non-wandering and each $\mathcal{G}_{i}$-orbit has length at least three, then the following are equivalent:

(1) $\mathcal{G}_{1} \cong \mathcal{G}_{2}$ as topological groupoids.

(2) $\llbracket \mathcal{G}_{1} \rrbracket \cong \llbracket \mathcal{G}_{2} \rrbracket$ as abstract groups.

Let us say a few words about the proofs. As the implications $(1) \Rightarrow(2) \Rightarrow(3)$ in Theorem $\mathrm{A}$ and $(1) \Rightarrow(2)$ in Theorem $\mathrm{B}$ are trivial, there is only one direction to prove. The proof strategy is similar in both cases and is summarized in the following diagram, ${ }^{\mathrm{c}}$ where $\Gamma_{i}$ is a subgroup of $\operatorname{Homeo}\left(\mathcal{G}_{i}^{(0)}\right)$ :

$$
\begin{array}{rlr}
\Gamma_{1} & \cong \Gamma_{2} & \text { (abstract isomorphism) } \\
\left(\Gamma_{1}, \mathcal{G}_{1}^{(0)}\right) & \cong\left(\Gamma_{2}, \mathcal{G}_{2}^{(0)}\right) \quad \text { (spatial isomorphism) } \\
& \| \text { Functoriality } \\
\operatorname{Germ}\left(\Gamma_{1}, \mathcal{G}_{1}^{(0)}\right) & \cong \operatorname{Germ}\left(\Gamma_{2}, \mathcal{G}_{2}^{(0)}\right) \\
& \| \Gamma_{i} \operatorname{covers} \mathcal{G}_{i} \\
\operatorname{Germ}\left(\Gamma_{1}, \mathcal{G}_{1}^{(0)}\right) \cong \mathcal{G}_{1} & \cong \mathcal{G}_{2} \cong \operatorname{Germ}\left(\Gamma_{2}, \mathcal{G}_{2}^{(0)}\right)
\end{array}
$$

The first step is showing that for certain classes of homeomorphism groups, any (abstract) group isomorphism is induced by a homeomorphism of the underlying spaces. We call this a spatial realization result. In [49], Matui proves a spatial realization result that applies to any $\Gamma$ with $\mathrm{D}(\llbracket \mathcal{G} \rrbracket) \leq \Gamma \leq \llbracket \mathcal{G} \rrbracket$ (for $\mathcal{G}$ minimal). And from a spatial isomorphism he directly constructs an isomorphism of the groupoids and obtains his Isomorphism Theorem. In this paper, we have chosen to break this direct step into two more parts in order to also study when the groupoid can be recovered from the action of (subgroups of) the topological full group on the unit space, as the groupoid of germs of this action. We find that such a groupoid of germs always embed into the groupoid we started with, and that they are isomorphic if and only if the subgroup in question is generated by enough bisections to cover the

${ }^{\mathrm{c}}$ If $\Gamma \leq \operatorname{Homeo}(X)$ and $\Lambda \leq \operatorname{Homeo}(Y)$ are groups of homeomorphisms, then a spatial isomorphism between them is a homeomorphism $\phi: X \rightarrow Y$ such that $\gamma \mapsto \phi \circ \gamma \circ \phi^{-1}$ for $\gamma \in \Gamma$ is a group isomorphism. 
groupoid (Proposition 4.10 Corollary 4.13). We also show that for a natural choice of maps, the assignment of the groupoid of germs is functorial (Proposition 5.4).

Having this machinery in place, proving Theorem $\mathrm{A}$ is then just a matter of checking that Matui's spatial realization result also holds in the locally compact setting (Theorem 6.6). Although this is but a small extension of Matui's result we have chosen to include it as a theorem since it is applicable to a larger class of groupoids. Regarding our initial motivation, namely the graph groupoids, we are able to characterize exactly when the aforementioned spatial realization result applies, and it turns out that we can get away with much weaker mixing properties than minimality when we restrict to graph groupoids - see Theorem C below.

For the proof of Theorem B we employ a spatial realization result (Theorem 6.19) based on Rubin's work in 62] in the first step. We mention that Medynets has previously obtained a similar spatial realization result [52, Remark 3] for (topological) full groups arising from group actions on the Cantor space, building on Fremlins work in [25, Sec. 384]. After some modifications, Theorem B could also be deduced from this result. However, Theorem 6.19 is more general as it can potentially be applied to other groups than topological full groups, e.g. homeomorphism groups of zero-dimensional linearly ordered spaces. See Remark 6.20 for a more detailed discussion on the differences and similarities of these approaches. Although Theorems $\mathrm{A}$ and $\mathrm{B}$ can be deduced by employing arguments along the lines of [49, 52, we believe that the way we trisect the proofs does add some new insight. In particular, this was how we discovered the embedding result given below in Theorem E.

Let us now describe the isomorphism theorem we obtain for graph groupoids, when starting with the spatial reconstruction result à la Matui. As mentioned above, it turns out that we can replace minimality (strong connectedness of the graphs) with some weaker "exit and return"-conditions. Each of these three conditions (see Definition 10.1) can be considered strengthenings of the three conditions that characterize when the boundary path space $\partial E$ has no isolated points (Proposition 8.1). Condition $(K)$ means that every cycle can be exited, and then returned to. Condition $(W)$ means that every wandering path can be exited, and then returned to. And Condition $(\infty)$ means that every singular vertex can be exited (i.e. is an infinite emitter), and then returned to (along infinitely many of the emitted edges).

Theorem C (cf. Theorem 10.10). Let $E$ and $F$ be graphs with no sinks, and suppose they both satisfy Condition $(K),(W)$ and $(\infty)$. Then the following are equivalent:

(1) $\mathcal{G}_{E} \cong \mathcal{G}_{F}$ as topological groupoids.

(2) $\llbracket \mathcal{G}_{E} \rrbracket \cong \llbracket \mathcal{G}_{F} \rrbracket$ as abstract groups.

(3) $\mathrm{D}\left(\llbracket \mathcal{G}_{E} \rrbracket\right) \cong \mathrm{D}\left(\llbracket \mathcal{G}_{F} \rrbracket\right)$ as abstract groups.

By interpreting the assumptions in Theorem B for graph groupoids, we obtain Theorem $\mathrm{D}$ below. Therein, Condition $(L)$ is the well-known exit condition of 
Kumjian, Pask and Raeburn [35, namely, that every cycle should have an exit. Condition (T) (see Definition 10.5) essentially means that the graph does not have a component which is a tree. Finally, what we call degenerate vertices (see Definition 10.6) are the ones giving $\mathcal{G}_{E}$-orbits of length one or two. This theorem may be considered a generalization of Matsumoto's result in the case of irreducible one-sided shifts of finite type [43] (which correspond to finite strongly connected graphs).

Theorem D (cf. Theorem 10.11). Let $E$ and $F$ be countable graphs satisfying Condition $(L)$ and $(T)$, and having no degenerate vertices. Then the following are equivalent:

(1) $\mathcal{G}_{E} \cong \mathcal{G}_{F}$ as topological groupoids.

(2) $\llbracket \mathcal{G}_{E} \rrbracket \cong \llbracket \mathcal{G}_{F} \rrbracket$ as abstract groups.

Hence, we establish the equivalence of (1)-(4) mentioned in the beginning of this subsection for graphs satisfying the assumptions of Theorem D In Corollary 10.13 we spell out this rigidity result for the associated graph algebras.

Our final main result is an embedding theorem for ample groupoids - inspired by embedding theorems for $C^{*}$-algebras and Leavitt path algebras. The seminal embedding theorem of Kirchberg [30] states that any separable exact (unital) $C^{*}$-algebra embeds (unitally) into the Cuntz algebra $\mathcal{O}_{2}$. In particular, this means that any graph $C^{*}$-algebra $C^{*}(E)$, where $E$ is a countable graph, embeds into $\mathcal{O}_{2}$. The latter, being the universal $C^{*}$-algebra generated by two orthogonal isometries, can be canonically identified with a graph $C^{*}$-algebra. Namely, the graph $C^{*}$-algebra of the graph $E_{2}$ which consists of a single vertex with two loops. In [1], Brownlowe and Sørensen show that the Leavitt path algebra $L_{R}(E)$, where $E$ is any countable graph and $R$ any commutative unital ring, embeds into $L_{R}\left(E_{2}\right)$ - the algebraic analog of $\mathcal{O}_{2}$. An inspection of their proof reveals that this embedding also maps the canonical diagonal subalgebra $D_{R}(E)$ into $D_{R}\left(E_{2}\right)$. As a consequence, Kirchberg's embedding for the graph $C^{*}$-algebras may then also be taken to be diagonal preserving - with respect to the diagonal ${ }^{\mathrm{d}}$ in $\mathcal{O}_{2}$ coming from its identification with $C^{*}\left(E_{2}\right)$. At this point, it starts smelling a bit like groupoids might be lurking about. Indeed, using the properties of the Germ-functor (see Sec. 5I), we are able to prove that the underlying graph groupoid $\mathcal{G}_{E}$ embeds into the Cuntz groupoid $\mathcal{G}_{E_{2}}$ (modulo topological obstructions in the sense of isolated points). Thus, the known embeddings of the graph algebras actually occur at the level of the underlying groupoid models. We were also able to extend this embedding result to all groupoids which are groupoid equivalent (or stably isomorphic) to a graph

\footnotetext{
d Technically, this is a Cartan subalgebra in the sense of Renault, not a $C^{*}$-diagonal in the sense of Kumjian. But it's common to refer to it as "the diagonal" in a graph $C^{*}$-algebra.
} 
groupoid. To the best of the authors' knowledge, this is the first embedding result of its kind for ample groupoids.

Theorem E (cf. Theorem 11.16). Let $\mathcal{H}$ be an effective ample second countable Hausdorff groupoid with $\mathcal{H}^{(0)}$ a locally compact Cantor space. If $\mathcal{H}$ is groupoid equivalent to $\mathcal{G}_{E}$, for some countable graph $E$ satisfying Condition $(L)$ and having no sinks nor semi-tails, then $\mathcal{H}$ embeds into $\mathcal{G}_{E_{2}}$. Moreover, if $\mathcal{H}^{(0)}$ is compact, then the embedding maps $\mathcal{H}^{(0)}$ onto $E_{2}^{\infty}$.

In particular, any graph groupoid $\mathcal{G}_{E}$, with $E$ as above, embeds into $\mathcal{G}_{E_{2}}$, and any AF-groupoid (with perfect unit space) embeds into $\mathcal{G}_{E_{2}}$.

The main ingredient in the proof is constructing an injective local homeomorphism $\phi: \partial E \rightarrow E_{2}^{\infty}$ which induces a spatial embedding of the associated topological full groups. This construction is entirely explicit. As a consequence, we also obtain explicit embeddings of any graph $C^{*}$-algebra $C^{*}(E)$ (or Leavitt path algebra $L_{R}(E)$ ), in terms of their canonical generators, into $\mathcal{O}_{2}$ (or $L_{R}\left(E_{2}\right)$ ). This embedding is diagonal preserving, and when $C^{*}(E)$ is unital (i.e. $E^{0}$ is finite) this embedding is unital and maps the diagonal onto the diagonal. These embeddings are described in Corollary 11.5] and Remark 11.6 We also record a result on diagonal embeddings of AF-algebras in Corollary 11.27.

Another consequence of Theorem $\mathrm{E}$ is that the topological full group $\llbracket \mathcal{G}_{E} \rrbracket$, for any graph $E$ as above, embeds into Thompson's group $V$ - since $V$ is isomorphic to $\llbracket \mathcal{G}_{E_{2}} \rrbracket$. The Higman-Thompson groups $V_{n, r}$ (where $\left.n V=V_{n, 1}\right)$ can be realized as topological full groups of graph groupoids of certain strongly connected finite graphs (see Sec. 11.3). Hence, our embedding theorem may be considered a generalization of the well-known embedding of $V_{n, r}$ into $V$. The embedding entails that the topological full groups $\llbracket \mathcal{H} \rrbracket$, of groupoids $\mathcal{H}$ as in Theorem $\mathrm{E}$ has the Haagerup property (but they are generally not amenable). In terms of groups, our embedding also includes all the so-called LDA-groups (see Remark 11.24).

In 50], Matui introduced two conjectures for minimal ample groupoids over the Cantor space. The $H K$-conjecture relates the groupoid homology to the $K$-theory of the groupoid $C^{*}$-algebra. And the $A H$-conjecture relates the topological full group to the groupoid homology. These conjectures have been verified in several cases [51], in particular for (products of) graph groupoids arising from strongly connected finite graphs. For the more general graph groupoids studied in the present paper, the second named author will, together with Toke Meier Carlsen, attack these conjectures in a forthcoming paper. (In the recent preprint [55], the second named author verifies the HK-conjecture for a class of groupoids which includes the graph groupoids of row-finite graphs.)

\section{Précis}

The structure of the paper is as follows. We recall some basic notions regarding étale groupoids and (classical) Stone duality in Sec. 2. This section also serves the 
purpose of establishing notation and conventions. The rest of the paper is divided into two parts. The first, Secs. [3] 1] deals with ample groupoids in general, while the second, Secs. 811, deals with graph groupoids.

In Sec. [3, we give the definition of the topological full group $\llbracket \mathcal{G} \rrbracket$ of an ample groupoid $\mathcal{G}$ with locally compact unit space $\mathcal{G}^{(0)}$. We also prove some elementary results on the existence of elements in the topological full group with certain properties. Then, we move on to study the groupoid of germs $\operatorname{Germ}\left(\Gamma, \mathcal{G}^{(0)}\right)$ associated to a subgroup $\Gamma \leq \llbracket \mathcal{G} \rrbracket$ of the topological full group, in Sec. 4. We establish that $\operatorname{Germ}\left(\Gamma, \mathcal{G}^{(0)}\right)$ always embeds into $\mathcal{G}$, and that this embedding is an isomorphism as long as $\Gamma$ contains "enough elements". In Sec. 5 we introduce the two categories; SpatG and Gpoid. The former consists of pairs $(\Gamma, X)$ where $X$ is a space and $\Gamma$ is a subgroup of $\operatorname{Homeo}(X)$. The latter consists of certain ample groupoids. By defining suitable morphisms in these categories and what the germ of a morphism in SpatG should be, we establish that the assignment $(\Gamma, X) \mapsto \operatorname{Germ}(\Gamma, X)$ is functorial. We also show that monomorphisms in SpatG induce étale embeddings of the associated groupoids of germs.

The spatial realization results needed to deduce that an abstract isomorphism of two topological full groups always is spatially implement are provided in Sec. 6] In Sec. [7, we prove the two general isomorphism theorems, Theorems $\mathrm{A}$ and $\mathrm{B}$ This is now mostly a matter of interpreting the spatial realization results from Sec. 6] in terms of the groupoid and its topological full group, and then combine this with the results of Secs. 4 and 5 ,

In Sec. 8, we begin our in-depth study of graph groupoids $\mathcal{G}_{E}$ of general graphs $E$. This section is devoted to a thorough introduction of graph terminology and the dynamics that give rise to the graph groupoids. For several of the generic properties a topological groupoid can have, we list their characterizations for graph groupoids in terms of the graphs. We continue in Sec. 9 with describing explicitly all elements in the topological full group $\llbracket \mathcal{G}_{E} \rrbracket$ of any graph groupoid. To do this we need to specify a new (yet equivalent) basis for the topology on $\mathcal{G}_{E}$. We then pursue specialized isomorphism theorems for the class of graph groupoids in Sec. 10 This yields Theorems $\mathrm{C}$ and D At the end of this section, we spell out the induced rigidity result for the associated graph algebras.

In the final section of the paper, we employ the machinery from Secs. 4, 5and 9 to obtain our groupoid embedding result; Theorem [E] We also describe the explicit diagonal embeddings of the graph algebras that follow from the embedding of the groupoids. Examples of these embeddings for graph algebras are provided for several infinite graphs. At the end of Sec. 11, we show that any AF-groupoid is groupoid equivalent to a graph groupoid, going via Bratteli diagrams, hence $\mathcal{G}_{E_{2}}$-embeddable. We then spell out consequences for diagonal embeddings of AF-algebras. Additionally, we remark that transformation groupoids arising from locally compact (noncompact) Cantor minimal systems are AF-groupoids, and hence $\mathcal{G}_{E_{2}}$-embeddable as well. 


\section{Preliminaries}

We will now recall the basic notions needed throughout the paper, as well as establish notation and conventions. We denote the positive integers by $\mathbb{N}$ and the nonnegative integers by $\mathbb{N}_{0}$. If two sets $A$ and $B$ are disjoint we will denote their union by $A \sqcup B$ if we wish to emphasize that they are disjoint. When we write $C=A \sqcup B$, we mean that $C=A \cup B$ and that $A$ and $B$ are disjoint sets.

\subsection{Topological notions}

Following [33, 65, we say that a topological space is Boolean if it is Hausdorff and has a basis of compact open sets. (This is also the terminology originally used by Stone [66].) A Stone space is then a compact Boolean space. We say that a topological space is perfect if it has no isolated points. By a locally compact Cantor space we mean a (non-empty) second countable perfect Boolean space. Up to homeomorphism there are two such spaces; one compact (the Cantor set) and one non-compact (the Cantor set with a point removed). The latter may also be realized as any non-closed open subset of the Cantor set, or as the product of the Cantor set and a countably infinite discrete space.

For a topological space $X$, we denote the group of self-homeomorphisms of $X$ by Homeo $(X)$. We will occasionally denote $\mathrm{id}_{X}$ simply by 1 for brevity. By an involution we mean a homeomorphism (or more generally, a group element) $\phi$ with $\phi^{2}=1$. For a homeomorphism $\phi \in \operatorname{Homeo}(X)$, we define the support of $\phi$ to be the (regular) closed set $\overline{\{x \in X \mid \phi(x) \neq x\}}$, and denote it by $\operatorname{supp}(\phi)$. We also define

$$
\operatorname{Homeo}_{c}(X):=\{\phi \in \operatorname{Homeo}(X) \mid \operatorname{supp}(\phi) \text { compact open }\} \text {. }
$$

When $\Gamma$ is a subgroup of a group $\Gamma^{\prime}$ we write $\Gamma \leq \Gamma^{\prime}$. Beware that we will abuse this notation when we write $\Gamma \leq \operatorname{Homeo}_{c}(X)$ to mean that $\Gamma$ is a subgroup of $\operatorname{Homeo}(X)$ and that $\Gamma \subseteq \operatorname{Homeo}_{c}(X)$. (It is not clear whether $\operatorname{Homeo}_{c}(X)$ itself is a group.)

\subsection{Stone duality}

We will now briefly recall the basics of (classical) Stone duality needed in Sec. 6. For more details the reader may consult [31, [25, Chap. 31] (or even the fountainhead [20, 66]). By a Boolean algebra we mean a complemented distributive lattice with a top and bottom element. And by a generalized Boolean algebra we mean a relatively complemented distributive lattice with a bottom element. For a topological space $X$, we denote the set of clopen subsets of $X$ by $\operatorname{CO}(X)$. The set of compact open subsets of $X$ are denoted by $\mathrm{CK}(X)$. Finally, the set of regular open subsets of $X$ are denoted by $\mathcal{R}(X)$. 
Example 2.1. Let $X$ be a topological space.

(1) $\mathrm{CO}(X)$ is a Boolean algebra under the operations of set-theoretic union, intersection and complement by $X$.

(2) $\mathrm{CK}(X)$ is a generalized Boolean algebra in the same way as $\mathrm{CO}(X)$, except for admitting only relative (set-theoretic) complements.

(3) $\mathcal{R}(X)$ is a Boolean algebra with the following operations. Let $A, B \in \mathcal{R}(X)$. The join of $A$ and $B$ is $(\overline{A \cup B})^{\circ}$, where o denotes the interior. The meet of $A$ and $B$ is $A \cap B$. And the complement of $A$ is $\sim A:=(X \backslash A)^{\circ}$.

A crude way of stating Stone duality is to say that every Boolean algebra arises as $\mathrm{CO}(X)$ for some Stone space $X$, and that every generalized Boolean algebra arises as $\mathrm{CK}(Y)$ for some Boolean space $Y$. Hence, Stone spaces correspond to Boolean algebras and Boolean spaces correspond to generalized Boolean algebras.

More precisely, it is a duality in the following sense. A continuous map $f: X \rightarrow Y$ between topological spaces $X$ and $Y$ is proper if $f^{-1}(K)$ is compact in $X$ whenever $K$ is a compact subset of $Y$. A map $\psi: \mathcal{A} \rightarrow \mathcal{B}$ between generalized Boolean algebras $\mathcal{A}$ and $\mathcal{B}$ is a Boolean homomorphism if it preserves joins, meets and relative complements. We say that $\psi$ is proper if for each $b \in \mathcal{B}$, there exists $a \in \mathcal{A}$ such that $\psi(a) \geq b$. Boolean spaces with proper continuous maps form a category. So does generalized Boolean algebras with proper Boolean homomorphisms. For a proper continuous map $f: X \rightarrow Y$, let $\mathrm{CK}(f)(A):=f^{-1}(A)$ for $A \in \mathrm{CK}(Y)$. This makes $\mathrm{CK}(-)$ a contravariant functor from the category of Boolean spaces to the category of generalized Boolean algebras (with maps as above).

For a generalized Boolean algebra $\mathcal{A}$, let $\mathbb{S}(\mathcal{A})$ denote the set of ultrafilters in $\mathcal{A}$. For each $a \in \mathcal{A}$, let $\mathbb{S}(a):=\{\alpha \in \mathbb{S}(\mathcal{A}) \mid a \in \alpha\}$. Equipping $\mathbb{S}(\mathcal{A})$ with the topology generated by the (compact open) cylinder sets $\mathbb{S}(a)$ turns it into a Boolean space. For a proper Boolean homomorphism $\psi: \mathcal{A} \rightarrow \mathcal{B}$ and an ultrafilter $\beta \in \mathbb{S}(\mathcal{B})$, let $\mathbb{S}(\psi)(\beta):=\left\{\psi^{-1}(b) \mid b \in \beta\right\}$. This makes $\mathbb{S}(-)$ a contravariant functor in the other direction, and we refer to it as the Stone functor. Stone duality asserts that the contravariant functors $\mathrm{CK}(-)$ and $\mathbb{S}(-)$ implement a dual equivalence. In other words, the category of Boolean spaces is dually equivalent to the category of generalized Boolean algebras. It is more common to state Stone duality in terms of Stone spaces and Boolean algebras. This is just the restriction of the duality above to the aforementioned sub-categories.

For a generalized Boolean algebra $\mathcal{A}$, we let $\operatorname{Aut}(\mathcal{A})$ denote the group of Boolean isomorphisms from $\mathcal{A}$ to $\mathcal{A}$.

\section{3. Étale groupoids}

The standard references for étale groupoids (and their $C^{*}$-algebras) are Renault's thesis [59] and Paterson's book [56]. See also the excellent lecture notes by Sims [64]. A groupoid is a small category of isomorphisms, that is, a set $\mathcal{G}$ (the 
morphisms, or arrows in the category) equipped with a partially defined multiplication $\left(g_{1}, g_{2}\right) \mapsto g_{1} \cdot g_{2}$ for a distinguished subset $\mathcal{G}^{(2)} \subseteq \mathcal{G} \times \mathcal{G}$, and everywhere defined involution $g \mapsto g^{-1}$ satisfying the following axioms:

(1) If $g_{1} g_{2}$ and $\left(g_{1} g_{2}\right) g_{3}$ are defined, then $g_{2} g_{3}$ and $g_{1}\left(g_{2} g_{3}\right)$ are defined and $\left(g_{1} g_{2}\right) g_{3}=g_{1}\left(g_{2} g_{3}\right)$,

(2) The products $g g^{-1}$ and $g^{-1} g$ are always defined. If $g_{1} g_{2}$ is defined, then $g_{1}=$ $g_{1} g_{2} g_{2}^{-1}$ and $g_{2}=g_{1}^{-1} g_{1} g_{2}$.

A topological groupoid is a groupoid equipped with a topology making the operations of multiplication and taking inverse continuous. The elements of the form $g g^{-1}$ are called units. We denote the set of units of a groupoid $\mathcal{G}$ by $\mathcal{G}^{(0)}$, and refer to this as the unit space. We think of the unit space as a topological space equipped with the relative topology from $\mathcal{G}$. The source and range maps are

$$
s(g):=g^{-1} g \quad \text { and } \quad r(g):=g g^{-1}
$$

for $g \in \mathcal{G}$. These maps are necessarily continuous when $\mathcal{G}$ is a topological groupoid. We implicitly assume that all unit spaces appearing are of infinite cardinality (in order to avoid some degenerate cases). An étale groupoid is a topological groupoid where the range map (and necessarily also the source map) is a local homeomorphism (as a map from $\mathcal{G}$ to $\mathcal{G}$ ). The unit space $\mathcal{G}^{(0)}$ of an étale groupoid is always an open subset of $\mathcal{G}$. An ample groupoid is an étale groupoid whose unit space is a Boolean space.

It is quite common for operator algebraists to restrict to Hausdorff groupoids. One reason for this is that a topological groupoid is Hausdorff if and only if the unit space is a closed subset of the groupoid. In the end our main results will only apply to groupoids that are Hausdorff, but some of the theory applies when $\mathcal{G}$ is merely ample (and effective). For as long as the unit space $\mathcal{G}^{(0)}$ is Hausdorff the groupoid will be locally Hausdorff. We shall therefore clearly indicate whenever we actually need the groupoid to be Hausdorff for some result to hold.

Two units $x, y \in \mathcal{G}^{(0)}$ belong to the same $\mathcal{G}$-orbit if there exists $g \in \mathcal{G}$ such that $s(g)=x$ and $r(g)=y$. We denote by $\operatorname{Orb}_{\mathcal{G}}(x)$ the $\mathcal{G}$-orbit of $x$. When every $\mathcal{G}$-orbit is dense in $\mathcal{G}^{(0)}, \mathcal{G}$ is called minimal. In the special case that there is just one orbit, we call $\mathcal{G}$ transitive. A subset $A \subseteq \mathcal{G}^{(0)}$ is called $\mathcal{G}$-full if $r\left(s^{-1}(A)\right)=\mathcal{G}^{(0)}$, in other words if $A$ meets every $\mathcal{G}$-orbit. For an open subset $A \subseteq \mathcal{G}^{(0)}$ the subgroupoid $\mathcal{G}_{\mid A}:=\{g \in \mathcal{G} \mid s(g), r(g) \in A\}$ is called the restriction of $\mathcal{G}$ to $A$. When $\mathcal{G}$ is étale, the restriction $\mathcal{G}_{\mid A}$ is an open étale subgroupoid. The isotropy group of a unit $x \in \mathcal{G}^{(0)}$ is the group $\mathcal{G}_{x}^{x}:=\{g \in \mathcal{G} \mid s(g)=r(g)=x\}$, and the isotropy bundle is

$$
\mathcal{G}^{\prime}:=\{g \in \mathcal{G} \mid s(g)=r(g)\}=\bigsqcup_{x \in \mathcal{G}^{(0)}} \mathcal{G}_{x}^{x} .
$$

A groupoid $\mathcal{G}$ is said to be principal if $\mathcal{G}^{\prime}=\mathcal{G}^{(0)}$, i.e. if all isotropy groups are trivial. Any principal groupoid can be identified with an equivalence relation on its unit 
space $\mathcal{G}^{(0)}$, but the topology need not be the relative topology from $\mathcal{G}^{(0)} \times \mathcal{G}^{(0)}$. We say that $\mathcal{G}$ is effective if the interior of $\mathcal{G}^{\prime}$ equals $\mathcal{G}^{(0)}$. We call $\mathcal{G}$ topologically principal if the set of points in $\mathcal{G}^{(0)}$ with trivial isotropy group are dense in $\mathcal{G}^{(0)}$.

Remark 2.2. We should point out that the condition we are calling effective often goes under the name essentially principal (or even topologically principal) elsewhere in the literature. In general, topologically principal implies effective. However, for most groupoids considered by operator algebraists the two notions are in fact equivalent (see [61. Proposition 3.1]), so often these names all mean the same thing. In particular, this is the case for second countable locally compact Hausdorff étale groupoids.

Definition 2.3. Let $\mathcal{G}$ be an étale groupoid. A bisection is an open subset $U \subseteq \mathcal{G}$ such that $s$ and $r$ are both injective when restricted to $U$. A bisection $U$ is called full if we have $s(U)=r(U)=\mathcal{G}^{(0)}$.

When $U$ is a bisection in $\mathcal{G}$, then $s_{\mid U}: U \rightarrow s(U)$ is a homeomorphism, and similarly for the range map. An étale groupoid can thus be characterized by admitting a topological basis consisting of bisections, and an ample groupoid as one with a basis of compact bisections. In particular, ample groupoids are locally compact, and if $\mathcal{G}$ is Hausdorff and ample, then $\mathcal{G}$ is also a Boolean space. One of the most basic class of examples of étale groupoids are the following, which arise from group actions.

Example 2.4. Let $\Gamma$ be a discrete group acting by homeomorphisms on a topological space $X$. The associated transformation groupoid is

$$
\Gamma \ltimes X:=\Gamma \times X
$$

with product according to $(\tau, \gamma(x)) \cdot(\gamma, x)=(\tau \gamma, x)$ (and undefined otherwise), and inverse $(\gamma, x)^{-1}=\left(\gamma^{-1}, \gamma(x)\right)$. Identifying the unit space $(\Gamma \ltimes X)^{(0)}=\{1\} \times X$ with $X$ in the obvious way we have $s((\gamma, x))=x$ and $r((\gamma, x))=\gamma(x)$. Equipping $\Gamma \ltimes X$ with the product topology makes it an étale groupoid (essentially because $\Gamma$ is discrete), and a basis of bisections is given by the cylinder sets

$$
Z(\gamma, U):=\{(\gamma, x) \mid x \in U\}
$$

indexed over $\gamma \in \Gamma$ and open subsets $U \subseteq X$. The identification of $X$ with the unit space as above is compatible with this topology. In particular $\Gamma \ltimes X$ is Hausdorff and ample exactly when $X$ is Boolean, and second countable when $\Gamma$ is countable and $X$ is second countable. The transformation groupoid is effective if and only if every nontrivial group element has support equal to $X$. In the second countable setting, this coincides with the action being topologically principal (meaning that the set of points that are fixed only by the identity element of the group form a 
dense subset of $X)$. The groupoid orbit $\operatorname{Orb}_{\Gamma \ltimes X}(x)$ of a point $x \in X$ coincide with the orbit under the action, i.e. $\operatorname{Orb}_{\Gamma \ltimes X}(x)=\{\gamma(x) \mid \gamma \in \Gamma\}=\operatorname{Orb}_{\Gamma \curvearrowright X}(x)$.

A groupoid homomorphism between two groupoids $\mathcal{G}$ and $\mathcal{H}$ is a map $\Phi: \mathcal{G} \rightarrow \mathcal{H}$ such that $\left(\Phi(g), \Phi\left(g^{\prime}\right)\right) \in \mathcal{H}^{(2)}$ whenever $\left(g, g^{\prime}\right) \in \mathcal{G}^{(2)}$, and moreover $\Phi(g) \cdot \Phi\left(g^{\prime}\right)=\Phi\left(g \cdot g^{\prime}\right)$. It follows that $\Phi\left(g^{-1}\right)=\Phi(g)^{-1}$ for all $g \in \mathcal{G}$, $\Phi$ commutes with the source and range maps and $\Phi\left(\mathcal{G}^{(0)}\right) \subseteq \mathcal{H}^{(0)}$. If $\Phi$ is a bijection, then $\Phi^{-1}$ is a groupoid homomorphism and we call $\Phi$ an algebraic isomorphism. For étale groupoids $\mathcal{G}$ and $\mathcal{H}$ an étale homomorphism is a groupoid homomorphism $\Phi: \mathcal{G} \rightarrow \mathcal{H}$ which is also a local homeomorphism. It is a fact that a groupoid homomorphism $\Phi: \mathcal{G} \rightarrow \mathcal{H}$ between étale groupoids is a local homeomorphism if and only if the restriction $\Phi^{(0)}: \mathcal{G}^{(0)} \rightarrow \mathcal{H}^{(0)}$ to the unit spaces is a local homeomorphism. By an isomorphism of topological (or étale) groupoids we mean an algebraic isomorphism which is also a homeomorphism. So a bijective étale homomorphism is an isomorphism of étale groupoids. Note that if $\Phi: \mathcal{G} \rightarrow \mathcal{H}$ is an étale homomorphism, then the image $\Phi(\mathcal{G})$ is an open étale subgroupoid of $\mathcal{H}$.

\section{The Topological Full Group}

In this section, we will expand Matui's definition of the topological full group of an ample groupoid from the compact to the locally compact case, and establish some elementary properties. To each bisection $U \subseteq \mathcal{G}$ in an étale groupoid, we associate a homeomorphism

$$
\pi_{U}: s(U) \rightarrow r(U)
$$

given by $r_{\mid U} \circ\left(s_{\mid U}\right)^{-1}$. This means that for each $g \in U, \pi_{U}$ maps $s(g)$ to $r(g)$. Whenever $U$ is a full bisection, $\pi_{U}$ is a homeomorphism of $\mathcal{G}^{(0)}$. We now show that the (partial) homeomorphism $\pi_{U}$ determines the bisection $U$, when the groupoid is effective and Hausdorff.

Lemma 3.1. Let $\mathcal{G}$ be an effective ample Hausdorff groupoid and let $U, V \subseteq \mathcal{G}$ be bisections with $s(U)=s(V)$ and $r(U)=r(V)$. If $\pi_{U}=\pi_{V}$, then $U=V$.

Proof. That $\pi_{U}=\pi_{V}$ means that for each $x \in s(U)$, the unique elements $g \in U$ and $h \in V$ with $s(g)=x=s(h)$ also satisfies $r(g)=r(h)$. This implies that $V^{-1} U \subseteq \mathcal{G}^{\prime}$. As $\mathcal{G}$ is Hausdorff, $\mathcal{G}^{(0)}$ is closed, and therefore $V^{-1} U \cap\left(\mathcal{G} \backslash \mathcal{G}^{(0)}\right)$ is an open subset of $\mathcal{G}^{\prime} \backslash \mathcal{G}^{(0)}$. But since $\mathcal{G}$ is effective this set must be empty. This entails that $V^{-1} U \subseteq \mathcal{G}^{(0)}$, and hence $U=V$.

Definition 3.2. Let $\mathcal{G}$ be an effective ample groupoid. The topological full group of $\mathcal{G}$, denoted $\llbracket \mathcal{G} \rrbracket$, is the subgroup of $\operatorname{Homeo}\left(\mathcal{G}^{(0)}\right)$ consisting of all homeomorphisms of the form $\pi_{U}$, where $U$ is a full bisection in $\mathcal{G}$ such that $\operatorname{supp}\left(\pi_{U}\right)$ is compact. We will denote by $\mathrm{D}(\llbracket \mathcal{G} \rrbracket)$ its commutator subgroup. 
In the topological full group, composition and inversion of the homeomorphisms correspond to multiplication and inversion of the bisections, viz.:

- $\pi_{\mathcal{G}^{(0)}}=\operatorname{id}_{\mathcal{G}^{(0)}}=1$,

- $\pi_{U} \circ \pi_{V}=\pi_{U V}$,

- $\left(\pi_{U}\right)^{-1}=\pi_{U^{-1}}$.

Remark 3.3. It is clear that when the unit space is compact, this definition coincides with Matui's [48, Definition 2.3] — which again generalizes the definitions given in 27 42, for Cantor dynamical systems and one-sided shifts of finite type, respectively, to étale groupoids. Moreover, in [46] Matui defined six different full groups associated with a minimal homeomorphism $\phi$ of a locally compact Cantor space. The smallest one of these, denoted $\tau[\phi]_{c}$ in $[46$, equals the topological full group (as in Definition 3.2) of the associated transformation groupoid.

Remark 3.4. After the completion of this work, we were made aware of Matte Bon's preprint [45] where he defines the topological full group of an arbitrary étale groupoid $\mathcal{G}$ as the group of all full bisections $U \subseteq \mathcal{G}$ such that $U \backslash \mathcal{G}^{(0)}$ is compact. For effective groupoids, this agrees with Definition 3.2 modulo identifying a full bisection with its associated homeomorphism. For not necessarily effective groupoids it is arguably better to define the topological full group in terms of the bisections themselves, for then one does not "lose" the information contained in the (nontrivial) isotropy (but also to separate the group from its canonical - no longer faithful — action on the unit space). This is done in e.g. [7, 54] as well. However, the approach taken in this paper — in particular in Sec. 6 — is based on working with subgroups of the homeomorphism group of a space (i.e. faithful group actions), which is why we have defined $\llbracket \mathcal{G} \rrbracket$ in terms of homeomorphisms.

Remark 3.5. We emphasize that the topological full group $\llbracket \mathcal{G} \rrbracket$ is viewed as a discrete group. The term topological is historical, and refers to the fact that the homeomorphisms in the topological full group preserves orbits in a "continuous way", as opposed to the full groups, which appeared first (in the measurable setting) cf. [27, p. 2].

For descriptions of the topological full group in certain classes of examples, see Proposition 9.4, Remarks 11.22 and 11.28. See also [51 for a survey on topological full groups of étale groupoids with compact unit space.

By virtue of the groupoid being effective, the support of a homeomorphism in the topological full group is in fact open as well. Matui's proof of this fact for compact unit spaces carries over verbatim to our setting.

Lemma 3.6 (cf. [49, Lemma 2.2]). Let $\mathcal{G}$ be an effective ample Hausdorff groupoid. Then $\operatorname{supp}\left(\pi_{U}\right)=s\left(U \backslash \mathcal{G}^{(0)}\right)$ for each $\pi_{U} \in \llbracket \mathcal{G} \rrbracket$. In particular, $\operatorname{supp}\left(\pi_{U}\right)$ is a compact open subset of $\mathcal{G}^{(0)}$. 
We now present a few basic results on the existence of elements in the topological full group. They will be used in later sections to construct elements in the topological full group with localized support.

Lemma 3.7. Let $\mathcal{G}$ be an effective ample groupoid, and let $\pi_{U} \in \llbracket \mathcal{G} \rrbracket$. Then, we have a decomposition

$$
U=U^{\perp} \sqcup\left(\mathcal{G}^{(0)} \backslash \operatorname{supp}\left(\pi_{U}\right)\right),
$$

where $U^{\perp}$ is a compact bisection with $s\left(U^{\perp}\right)=r\left(U^{\perp}\right)=\operatorname{supp}\left(\pi_{U}\right)$.

Conversely, any compact bisection $V \subseteq \mathcal{G}$ with $s(V)=r(V)$ defines an element $\pi_{\tilde{V}} \in \llbracket \mathcal{G} \rrbracket$ with $\operatorname{supp}\left(\pi_{\tilde{V}}\right) \subseteq s(V)$ by setting $\tilde{V}=V \sqcup\left(\mathcal{G}^{(0)} \backslash s(V)\right)$.

Proof. It is clear that $\operatorname{supp}\left(\pi_{U}\right)$ is invariant under $\pi_{U}$. Therefore, we may simply put $U^{\perp}=s_{\mid U}^{-1}\left(\operatorname{supp}\left(\pi_{U}\right)\right)$. The second statement is obvious.

Lemma 3.8. Let $\mathcal{G}$ be an effective ample groupoid. Any compact bisection $V \subseteq \mathcal{G}$ which satisfies $s(V) \cap r(V)=\emptyset$ defines an involution $\pi_{\hat{V}} \in \llbracket \mathcal{G} \rrbracket$ by setting $\hat{V}$ equal to $V \sqcup V^{-1} \sqcup\left(\mathcal{G}^{(0)} \backslash(s(V) \cup r(V))\right)$. Moreover, $\operatorname{supp}\left(\pi_{\hat{V}}\right) \subseteq s(V) \cup r(V)$.

Proof. Immediate.

Lemma 3.9. Let $\mathcal{G}$ be an effective ample groupoid. If $g \in \mathcal{G} \backslash \mathcal{G}^{\prime}$, that is $s(g) \neq r(g)$, then there is a (nontrivial) bisection $U \subseteq \mathcal{G}$ containing $g$ with $\pi_{U} \in \llbracket \mathcal{G} \rrbracket$. Furthermore, for any open set $A \subseteq \mathcal{G}^{(0)}$ containing both $s(g)$ and $r(g), U$ can be chosen so that $\operatorname{supp}\left(\pi_{U}\right) \subseteq A$. We may also choose $\pi_{U}$ to be an involution.

Proof. As $\mathcal{G}$ is ample there is a compact bisection $W$ containing $g$. Let $B_{1}, B_{2}$ be disjoint open neighborhoods of $s(g), r(g)$, respectively, in $\mathcal{G}^{(0)}$. By intersecting we may take $B_{1} \subseteq s(W) \cap A$ and $B_{2} \subseteq r(W) \cap A$. By continuity of $s$ and $r$ there are compact open sets $W_{1}, W_{2} \subseteq W$, both containing $g$, such that $s\left(W_{1}\right) \subseteq B_{1}$ and $r\left(W_{2}\right) \subseteq B_{2}$. And then $V=W_{1} \cap W_{2}$ is a compact bisection containing $g$ with $s(V) \cap r(V)=\emptyset$ and $s(V) \cup r(V) \subseteq A$. Hence $U=\hat{V}$ (as in Lemma 3.8) is the desired full bisection.

Remark 3.10. In the non-compact case, we may view the topological full group as a direct limit of topological full groups of groupoids over compact spaces as follows. Consider $\operatorname{CK}\left(\mathcal{G}^{(0)}\right)$ as a directed set (ordered by inclusion). Given $A, B \in \operatorname{CK}\left(\mathcal{G}^{(0)}\right)$ with $A \subseteq B$ we define the group homomorphism $\iota_{A, B}: \llbracket \mathcal{G}_{A} \rrbracket \rightarrow \llbracket \mathcal{G}_{B} \rrbracket$ by $\pi_{U} \mapsto \pi_{\tilde{U}}$ where we set $\tilde{U}=U \sqcup(B \backslash A)$. Then, we have that

$$
\llbracket \mathcal{G} \rrbracket \cong \underset{\lim }{\longrightarrow}\left(\llbracket \mathcal{G}_{A} \rrbracket, \iota\right) .
$$




\section{The Groupoid of Germs}

We are now going to adapt the notions of [61, Sec. 3] to the (special) case of groups, rather than inverse semigroups, to fit the framework of the topological full group and its subgroups, rather than the pseudogroup studied in [61]. Our goal is to reconstruct an ample groupoid $\mathcal{G}$ from subgroups of the topological full group $\llbracket \mathcal{G} \rrbracket$ as a so-called groupoid of germs - which is a quotient of a transformation groupoid.

Remark 4.1. In the following three sections, we will be working with subgroups of $\operatorname{Homeo}(X)$, where $X$ is a topological space. Thus, we are essentially studying faithful actions by discrete groups on $X$. In the end we will have $X=\mathcal{G}^{(0)}$ for some ample groupoid $\mathcal{G}$, and we will be looking at subgroups of $\llbracket \mathcal{G} \rrbracket$. Yet it will be convenient to state most results for general subgroups $\Gamma \leq \operatorname{Homeo}(X)$ without reference to groupoids. Also, beware that the term faithful will be used differently in Sec. 6 (cf. Definition 6.1).

Recall that two homeomorphisms $\gamma, \tau: X \rightarrow X$ have the same germ at the point $x \in X$ if there is a neighborhood $U$ of $x$ such that $\gamma_{\mid U}=\tau_{\mid U}$.

Definition 4.2. Let $X$ be a locally compact Hausdorff space and let $\Gamma$ be a subgroup of $\operatorname{Homeo}(X)$. The groupoid of germs of $(\Gamma, X)$ is

$$
\operatorname{Germ}(\Gamma, X):=(\Gamma \ltimes X) / \sim,
$$

where $(\gamma, x) \sim(\tau, y)$ if and only if $x=y$ and $\gamma, \tau$ have the same germ at $x$.

Denote the equivalence class of $(\gamma, x) \in \Gamma \ltimes X$ under $\sim$ by $[\gamma, x]$. It is straightforward to check that the groupoid operations of the transformation groupoid are well-defined on representatives of the equivalence classes in the groupoid of germs (and that they are continuous). The bisections

$$
Z[\gamma, A]:=\{[\gamma, x] \mid x \in A\},
$$

for $\gamma \in \Gamma$ and $A \subseteq X$ open, form a basis for the quotient topology. The unit space of $\operatorname{Germ}(\Gamma, X)$ is also identified with $X$ in the obvious way. Hence $\operatorname{Germ}(\Gamma, X)$ is étale (and ample when $X$ is Boolean), and it is furthermore always effective (as any group element acting identically on an open set is identified with the identity at each point of this open set). Hausdorffness of the groupoid however, is no longer guaranteed, but it can be characterized as follows.

Lemma 4.3. Let $X$ be a locally compact Hausdorff space and let $\Gamma \leq \operatorname{Homeo}(X)$. Then the groupoid of germs $\operatorname{Germ}(\Gamma, X)$ is Hausdorff if and only if $\operatorname{supp}(\gamma)$ is clopen in $X$ for every $\gamma \in \Gamma$.

Proof. Since $X$ is Hausdorff, any two groupoid elements $[\gamma, x],[\tau, y] \in \operatorname{Germ}(\Gamma, X)$ with distinct sources (i.e. $x \neq y$ ) can always be separated by open sets. We only have to worry about separating elements in the same isotropy group, and it suffices 
to be able to separate the unit from any other element. Also note that $[\gamma, x] \neq[1, x]$ if and only if $x \in \operatorname{supp}(\gamma)$.

First, assume that all the supports are clopen. If $[\gamma, x] \neq[1, x]$, then by the observation above, $Z[\gamma, \operatorname{supp}(\gamma)]$ and $Z[1, \operatorname{supp}(\gamma)]$ are disjoint open neighborhoods of these elements. To separate $[\gamma, x]$ from $[\tau, x]$ (when these are distinct), we first note that $[\gamma, x][\tau, x]^{-1}=\left[\gamma \tau^{-1}, \tau(x)\right] \neq[1, \tau(x)]$. Hence $\tau(x) \in \operatorname{supp}\left(\gamma \tau^{-1}\right)$, so by the argument above $Z\left[\gamma \tau^{-1}, A\right]$ and $Z[1, A]$, with $A=\operatorname{supp}\left(\gamma \tau^{-1}\right)$, separates $\left[\gamma \tau^{-1}, \tau(x)\right]$ from $[1, \tau(x)]$. It follows that $Z\left[\gamma, \tau^{-1}(A)\right]$ and $Z\left[\tau, \tau^{-1}(A)\right]$ separates $[\gamma, x]$ and $[\tau, x]$.

Conversely, suppose there is a $\gamma \in \Gamma \operatorname{such}$ that $\operatorname{supp}(\gamma)$ is not open. Let $x$ be any point on the boundary of $\operatorname{supp}(\gamma)$. Then $\gamma(x)=x$, but $[\gamma, x] \neq[1, x]$, and these two groupoid elements cannot be separated by open sets. To see this take any two basic neighborhoods $Z[\gamma, A], Z[1, B]$ where $A, B$ are open neighborhoods of $x$ in $X$. They both contain the basic set $Z[1, C]$ where $C=(A \cap B) \backslash \operatorname{supp}(\gamma)$, since $\gamma$ acts identically on $C$.

In the sequel we shall restrict our attention to groups of homeomorphisms which have open, as well as compact, support. Topological full groups are determined by the "local behavior" of its elements. This is made precise in the following definition.

Definition 4.4. Let $X$ be a locally compact Hausdorff space and let $\Gamma$ be a subgroup of $\operatorname{Homeo}_{c}(X)$. We say that a homeomorphism $\varphi \in \operatorname{Homeo}_{c}(X)$ locally belongs to $\Gamma$ if for every $x \in X$, there exists an open neighborhood $U$ of $x$ and $\gamma \in \Gamma$ such that $\varphi_{\mid U}=\gamma_{\mid U}$. The group $\Gamma$ is called locally closed if whenever $\varphi \in \operatorname{Homeo}_{c}(X)$ locally belongs to $\Gamma$, then $\varphi \in \Gamma$.

Proposition 4.5. Let $\mathcal{G}$ be an effective ample Hausdorff groupoid. Then the topological full group $\llbracket \mathcal{G} \rrbracket \leq \operatorname{Homeo}_{c}\left(\mathcal{G}^{(0)}\right)$ is locally closed.

Proof. Let $\varphi \in \operatorname{Homeo}_{c}\left(\mathcal{G}^{(0)}\right)$ locally belong to $\llbracket \mathcal{G} \rrbracket$. Then, since $\operatorname{supp}(\varphi)$ is compact open, we can find finitely many open sets $A_{i} \subseteq \operatorname{supp}(\varphi)$, covering $\operatorname{supp}(\varphi)$, such that $\varphi_{\mid A_{i}}=\left(\pi_{U_{i}}\right)_{\mid A_{i}}$, where $\pi_{U_{i}} \in \llbracket \mathcal{G} \rrbracket$. Since $\mathcal{G}^{(0)}$ is Boolean we may assume that the $A_{i}$ 's are clopen and disjoint. We then have a clopen partition $\operatorname{supp}(\varphi)=A_{1} \sqcup A_{2} \sqcup \cdots \sqcup A_{n}$, and $\varphi$ restricts to a self-homeomorphism of $\operatorname{supp}(\varphi)$ which on each region $A_{i}$ equals $\pi_{U_{i}}$. It follows that the set $V=\bigcup_{i=1}^{n} V_{i}$, where $V_{i}=\left(s_{\mid U_{i}}\right)^{-1}\left(A_{i}\right)$, is a compact bisection in $\mathcal{G}$ with $s(V)=\operatorname{supp}(\varphi)=r(V)$. And then $\varphi=\pi_{\tilde{V}} \in \llbracket \mathcal{G} \rrbracket$, where $\tilde{V}$ is as in Lemma 3.7

Given a group $\Gamma \leq \operatorname{Homeo}_{c}(X)$ we denote by $\langle\Gamma\rangle$ the set of $\varphi \in \operatorname{Homeo}_{c}(X)$ which locally belong to $\Gamma$. Clearly $\langle\Gamma\rangle$ is a locally closed group in $\operatorname{Homeo}_{c}(X)$ and $\Gamma \leq\langle\Gamma\rangle$. As the groupoid of germs is defined in the same local terms as the local closure we have a canonical isomorphism $\operatorname{Germ}(\langle\Gamma\rangle, X) \cong \operatorname{Germ}(\Gamma, X)$. From 
this we obtain the analog of [61, Proposition 3.2], namely that the topological full group of a groupoid of germs equals the local closure of the group we started with.

Proposition 4.6. Let $X$ be a Boolean space and let $\Gamma \leq \operatorname{Homeo}_{c}(X)$. Then, we have that $\llbracket \operatorname{Germ}(\Gamma, X) \rrbracket \cong\langle\Gamma\rangle$.

Proof. Since $\operatorname{Germ}(\Gamma, X)$ is canonically isomorphic to $\operatorname{Germ}(\langle\Gamma\rangle, X)$, it suffices to show that $\llbracket \operatorname{Germ}(\langle\Gamma\rangle, X) \rrbracket=\langle\Gamma\rangle$. For each $\varphi \in\langle\Gamma\rangle$ the full bisection $Z[\varphi, X]=U_{\varphi}$ in $\operatorname{Germ}(\langle\Gamma\rangle, X)$ satisfies $\pi_{U_{\varphi}}=\varphi$. And since $\varphi$ has compact support it belongs to $\llbracket \operatorname{Germ}(\langle\Gamma\rangle, X) \rrbracket$.

For the reverse inclusion, take any $\pi_{U} \in \llbracket \operatorname{Germ}(\langle\Gamma\rangle, X) \rrbracket$. Recall that the support of $\pi_{U}$ is open, as well as compact, since any groupoid of germs is effective (cf. Lemma 3.6). To see that $\pi_{U}$ locally belongs to $\Gamma$ take any $x \in X$, and let $[\varphi, x]$ be the unique element in $U$ whose source is $x$. Since $U$ is open there is a basic set $Z[\varphi, A] \subseteq U$, where $A$ is an open neighborhood of $x$ in $X$. As $\varphi \in\langle\Gamma\rangle$ there is an open neighborhood $B$ of $x$ and an element $\gamma \in \Gamma$ with $\varphi_{\mid B}=\gamma_{\mid B}$. By intersecting with $A$ we may assume that $B \subseteq A$. Now observe that $\left(\pi_{U}\right)_{\mid B}=\varphi_{\mid B}=\gamma_{\mid B}$, and we are done.

As topological full groups are locally closed (Proposition 4.5), we obtain the following immediate corollary.

Corollary 4.7. Let $\mathcal{G}$ be an effective ample Hausdorff groupoid. Then, we have that $\llbracket \operatorname{Germ}\left(\llbracket \mathcal{G} \rrbracket, \mathcal{G}^{(0)}\right) \rrbracket \cong \llbracket \mathcal{G} \rrbracket$.

The preceding results show that a locally closed group $\Gamma \leq \operatorname{Homeo}_{c}(X)$ can be reconstructed from its associated groupoid of germs $\operatorname{Germ}\left(\Gamma, \mathcal{G}^{(0)}\right)$, namely as the topological full group of this groupoid. We now turn to the question of how an ample groupoid $\mathcal{G}$ relates to the groupoid of germs, $\operatorname{Germ}\left(\llbracket \mathcal{G} \rrbracket, \mathcal{G}^{(0)}\right)$, determined by its topological full group. We will see that these will also be isomorphic under some mild condition on the groupoid - namely that the groupoid can be covered by bisections as in the following definition.

Definition 4.8. Let $\mathcal{G}$ be an effective ample groupoid. We say that a subgroup $\Gamma \leq \llbracket \mathcal{G} \rrbracket$ covers $\mathcal{G}$ if there for each $g \in \mathcal{G}$ exists a $\pi_{U} \in \Gamma$ such that $g \in U$.

Note that if $\Gamma \leq \llbracket \mathcal{G} \rrbracket$ covers $\mathcal{G}$, then so does any group $\Gamma^{\prime}$ lying in between them, i.e. $\Gamma \leq \Gamma^{\prime} \leq \llbracket \mathcal{G} \rrbracket$, and in particular $\llbracket \mathcal{G} \rrbracket$ itself covers $\mathcal{G}$. Sufficient conditions on the orbits of $\mathcal{G}$ for $\llbracket \mathcal{G} \rrbracket$, or the commutator $\mathrm{D}(\llbracket \mathcal{G} \rrbracket)$, to cover $\mathcal{G}$ is given by the following result (which is the analog of [49, Lemma 3.7]).

Lemma 4.9. Let $\mathcal{G}$ be an effective ample groupoid.

(1) If $\left|\operatorname{Orb}_{\mathcal{G}}(x)\right| \geq 2$ for every $x \in \mathcal{G}^{(0)}$, then $\llbracket \mathcal{G} \rrbracket$ covers $\mathcal{G}$.

(2) If $\left|\operatorname{Orb}_{\mathcal{G}}(x)\right| \geq 3$ for every $x \in \mathcal{G}^{(0)}$, then $\mathrm{D}(\llbracket \mathcal{G} \rrbracket)$ covers $\mathcal{G}$. 
Proof. (1) First consider $g \in \mathcal{G} \backslash \mathcal{G}^{\prime}$. Then Lemma 3.9 immediately gives an element $\pi_{U} \in \llbracket \mathcal{G} \rrbracket$ with $g \in U$. Next, suppose $s(g)=r(g)=x$. By assumption there is a point $y$ different from $x$ in $\operatorname{Orb}_{\mathcal{G}}(x)$. This means that there is some $h \in \mathcal{G}$ with $s(h)=x \neq y=r(h)$. And then $h^{-1}$ is composable with $g$ and $g h^{-1} \in \mathcal{G} \backslash \mathcal{G}^{\prime}$. Applying Lemma 3.9 to both $g h^{-1}$ and $h$ we get $\pi_{U_{1}}, \pi_{U_{2}} \in \llbracket \mathcal{G} \rrbracket$ with $g h^{-1} \in U_{1}$ and $h \in U_{2}$. Since $\pi_{U_{1} U_{2}} \in \llbracket \mathcal{G} \rrbracket$ and $g \in U_{1} U_{2}$ we see that $\llbracket \mathcal{G} \rrbracket$ covers $\mathcal{G}$.

(2) As in the previous part we first consider the case $g \in \mathcal{G} \backslash \mathcal{G}^{\prime}$. By assumption there is a third (distinct) point $y$ in the same orbit as $s(g)$ and $r(g)$. Therefore there is an element $h \in \mathcal{G}$ with $s(h)=y$ and $r(h)=s(g)$. Lemma 3.9] gives involutions $\pi_{U}, \pi_{V} \in \llbracket \mathcal{G} \rrbracket$ such that $g \in U$ and $h \in V$. We may also arrange so that $y \notin \operatorname{supp}\left(\pi_{U}\right)$ by the second part of Lemma 3.9. Then

$$
\left[\pi_{U}, \pi_{V}\right]=\pi_{U} \pi_{V}\left(\pi_{U}\right)^{-1}\left(\pi_{V}\right)^{-1}=\pi_{(U V)^{2}} \in \mathrm{D}(\llbracket \mathcal{G} \rrbracket),
$$

and we claim that $g$ belongs to the associated full bisection $(U V)^{2}$. To see that this is the case, note that $y \in U$ since $y \notin \operatorname{supp}\left(\pi_{U}\right)$. Thus we have $g=g \cdot h \cdot y \cdot h^{-1} \in U V U V$ as $s(h)=y$.

Finally, for the case $s(g)=r(g)$ we proceed similar as in part (1). We take $h \in \mathcal{G}$ with $s(h)=s(g)$ and $r(h) \neq s(g)$ and apply the above part to $g h^{-1}$ and $h$, which both belong to $\mathcal{G} \backslash \mathcal{G}^{\prime}$. Multiplying the bisections we get gives the desired bisection containing $g$.

The conditions in Lemma 4.9 are not necessary (cf. Example 9.6), but they are typically easy to check in specific examples. Note that for minimal groupoids all orbits are in particular infinite, so the covering as above is automatic. We are now ready to give the main result on how a groupoid $\mathcal{G}$ can be reconstructed from the germs of $\llbracket \mathcal{G} \rrbracket$. It is the analog of [61, Proposition 3.2].

Proposition 4.10. Let $\mathcal{G}$ be an effective ample Hausdorff groupoid and let $\Gamma \leq \llbracket \mathcal{G} \rrbracket$. Then there is an injective étale homomorphism

$$
\iota: \operatorname{Germ}\left(\Gamma, \mathcal{G}^{(0)}\right) \hookrightarrow \mathcal{G}
$$

given by $\iota\left(\left[\pi_{U}, x\right]\right)=\left(s_{\mid U}\right)^{-1}(x)$ for $\left[\pi_{U}, x\right] \in \operatorname{Germ}\left(\Gamma, \mathcal{G}^{(0)}\right)$. Furthermore, $\iota$ is surjective, and hence an isomorphism, if and only if $\Gamma$ covers $\mathcal{G}$.

Proof. We first have to verify that $\iota$ is well-defined. Let $x \in \mathcal{G}^{(0)}$ and suppose that the homeomorphisms $\pi_{U}, \pi_{V} \in \Gamma$ have the same germ over $x$. Let $A$ be an open neighborhood of $x$ on which $\pi_{U}$ and $\pi_{V}$ agree. Then

$$
\pi_{U A}=\left(\pi_{U}\right)_{\mid A}=\left(\pi_{V}\right)_{\mid A}=\pi_{V A},
$$

so by Lemma 3.1 we have $U A=V A$. This means that the unique groupoid elements in $U$ and $V$ that have source equal to $x$ coincide, so $\iota$ is well-defined.

To see that $\iota$ is a groupoid homomorphism recall that $\left(\left[\pi_{V}, y\right],\left[\pi_{U}, x\right]\right)$ is a composable pair if and only if $\pi_{U}(x)=y$. Suppose this is the case and let 
$g \in U$ be the element with $s(g)=x$, and let $h \in V$ be the element with $s(h)=y$. As $r(g)=\pi_{U}(x)=y=s(h)$ we have $(h, g) \in \mathcal{G}^{(2)}$ and

$$
\iota\left(\left[\pi_{V}, y\right] \cdot\left[\pi_{U}, x\right]\right)=\iota\left(\left[\pi_{V U}, x\right]\right)=h g,
$$

since $h g \in V U$ and $s(h g)=x$.

Now note that $\iota(x)=x$ for $x \in \mathcal{G}^{(0)}$ (under the identification of the unit space of the groupoid of germs). So $\iota^{(0)}=\mathrm{id}_{\mathcal{G}^{(0)}}$ is a (local) homeomorphism, hence $\iota$ is an étale homomorphism.

To see that $\iota$ is injective note first that $\iota\left(\left[\pi_{U}, x\right]\right) \neq \iota\left(\left[\pi_{V}, y\right]\right)$ if $x \neq y$ since $\iota^{(0)}$ is the identity. Suppose now that $\iota\left(\left[\pi_{U}, x\right]\right)=\iota\left(\left[\pi_{V}, x\right]\right)$ for some $\pi_{U}, \pi_{V} \in \Gamma$. This means that there is a groupoid element $g \in U \cap V$ with $s(g)=x$. Thus $B=s(U \cap V)$ is an open neighborhood of $x$ in $\mathcal{G}^{(0)}$ and clearly $\left(\pi_{U}\right)_{\mid B}=\left(\pi_{V}\right)_{\mid B}$, which means that $\left[\pi_{U}, x\right]=\left[\pi_{V}, x\right]$.

Finally, that $\iota$ is surjective is easily seen to be the same as $\Gamma$ covering $\mathcal{G}$.

Remark 4.11. When the map $\iota$ in the previous proposition is an isomorphism the inverse is given by $\iota^{-1}(g)=\left[\pi_{U}, s(g)\right]$, where $U$ is any full bisection such that $\pi_{U} \in \Gamma$ and $g \in U$.

Remark 4.12. Let $\mathcal{G}$ be an effective ample Hausdorff groupoid. Combining Propositions 4.10 and 4.6 we see that for each locally closed subgroup $\Gamma \leq \llbracket \mathcal{G} \rrbracket$, there is an open étale subgroupoid $\mathcal{H}_{\Gamma} \subseteq \mathcal{G}$ such that $\llbracket \mathcal{H}_{\Gamma} \rrbracket \cong \Gamma$, namely $\mathcal{H}_{\Gamma}=\operatorname{Germ}\left(\Gamma, \mathcal{G}^{(0)}\right)$.

Since we are really interested in knowing when $\mathcal{G}$ is isomorphic to $\operatorname{Germ}\left(\Gamma, \mathcal{G}^{(0)}\right)$ (particularly for the case $\Gamma=\llbracket \mathcal{G} \rrbracket$ ) it is natural to ask whether they could be isomorphic even if the canonical map $\iota$ fails to be an isomorphism. We will see shortly that this is not possible. For $\Gamma \leq \operatorname{Homeo}_{c}(X)$ with $X$ Boolean we have seen that $\Gamma \leq\langle\Gamma\rangle \cong \llbracket \operatorname{Germ}(\Gamma, X) \rrbracket$. Identifying the latter two we see that $\Gamma$ covers $\operatorname{Germ}(\Gamma, X)$ since we have $[\gamma, x] \in Z[\gamma, X]$ and $\pi_{Z[\gamma, X]}=\gamma \in \Gamma$ for each $[\gamma, x] \in \operatorname{Germ}(\Gamma, X)$.

Corollary 4.13. Let $\mathcal{G}$ be an effective ample Hausdorff groupoid. Then $\mathcal{G}$ is isomorphic to $\operatorname{Germ}\left(\llbracket \mathcal{G} \rrbracket, \mathcal{G}^{(0)}\right)$ if and only if $\llbracket \mathcal{G} \rrbracket$ covers $\mathcal{G}$.

Proof. Suppose $\Phi: \mathcal{G} \rightarrow \operatorname{Germ}\left(\llbracket \mathcal{G} \rrbracket, \mathcal{G}^{(0)}\right)$ is an isomorphism. Then $\Phi$ induces an isomorphism between the topological full groups by $\pi_{U} \mapsto \pi_{\Phi(U)}$ for $\pi_{U} \in$ $\llbracket \mathcal{G} \rrbracket$. Let $g \in \mathcal{G}$ be given. As $\llbracket \mathcal{G} \rrbracket$ covers Germ $\left(\llbracket \mathcal{G} \rrbracket, \mathcal{G}^{(0)}\right)$ there is a full bisection $V$ containing $\Phi(g)$ such that $\pi_{V} \in \llbracket \operatorname{Germ}\left(\llbracket \mathcal{G} \rrbracket, \mathcal{G}^{(0)}\right) \rrbracket=\llbracket \mathcal{G} \rrbracket$. And then $\Phi^{-1}(V)$ is a full bisection in $\mathcal{G}$ containing $g$ with $\pi_{\Phi^{-1}(V)} \in \llbracket \mathcal{G} \rrbracket$. Hence $\llbracket \mathcal{G} \rrbracket$ covers $\mathcal{G}$.

\section{The Category of Spatial Groups}

In this section, we will study the groupoid of germs from a categorical point of view. We will see that the assignment $(\Gamma, X) \mapsto \operatorname{Germ}(\Gamma, X)$ is functorial - after 
introducing suitable categories. We will also see that certain equivariant maps between the spaces induce embeddings of the groupoids of germs.

Definition 5.1. The category of spatial groups, denoted by SpatG, consists of pairs $(\Gamma, X)$, where $X$ is a Boolean space and $\Gamma \leq \operatorname{Homeo}_{c}(X)$. A morphism in SpatG from $\left(\Gamma_{1}, X_{1}\right)$ to $\left(\Gamma_{2}, X_{2}\right)$ is a local homeomorphism $\phi: X_{1} \rightarrow X_{2}$ satisfying $\phi \circ \Gamma_{1} \subseteq \Gamma_{2} \circ \phi$.

We shall sometimes refer to a pair $(\Gamma, X)$ as a space-group pair. Observe that an isomorphism in the category SpatG is a homeomorphism $\phi$ such that $\phi \circ \Gamma_{1} \circ \phi^{-1}=\Gamma_{2}$. We call such an isomorphism a spatial isomorphism (as it is a group isomorphism implemented by a homeomorphism).

Definition 5.2. The category Gpoid consists of ample effective Hausdorff groupoids, and the morphisms are étale homomorphisms.

Remark 5.3. The choice of morphisms in SpatG is done so that they induce étale homomorphisms between the groupoid of germs in a natural way. As for the morphisms in Gpoid, there are several reasons for stipulating that they should be étale homomorphisms (rather than merely continuous groupoid homomorphisms). First of all, since all the structure maps in an étale groupoid are local homeomorphisms, it is reasonable to prescribe that maps between étale groupoids should be as well. Moreover, the image under an étale homomorphism is always an open étale subgroupoid in the codomain. An important consequence of this is that an injective étale homomorphism induce (diagonal preserving) injective $*$-homomorphisms between both the full and reduced groupoid $C^{*}$-algebras, respectively (and also between the Steinberg algebras), cf. [9, p. 113] and [57, Proposition 1.9]. Whereas the groupoid $C^{*}$-algebra construction is not functorial in general.

It is straightforward to check that SpatG and Gpoid indeed are categories. We will now define a functor from SpatG to Gpoid, which on objects is the groupoid of germs. Let $\phi$ be a spatial morphism between two space-group pairs $\left(\Gamma_{1}, X_{1}\right)$ and $\left(\Gamma_{2}, X_{2}\right)$ in SpatG. Given $[\gamma, x] \in \operatorname{Germ}\left(\Gamma_{1}, X_{1}\right)$, there is an element $\gamma^{\prime} \in \Gamma_{2}$ with $\phi \circ \gamma=\gamma^{\prime} \circ \phi$. We then propose to define an étale homomorphism $\operatorname{Germ}(\phi)$ from $\operatorname{Germ}\left(\Gamma_{1}, X_{1}\right)$ to $\operatorname{Germ}\left(\Gamma_{2}, X_{2}\right)$ by setting $\operatorname{Germ}(\phi)([\gamma, x])=\left[\gamma^{\prime}, \phi(x)\right]$.

Proposition 5.4. The mapping $\operatorname{Germ}(\phi)$ described above is a well-defined étale homomorphism, and $\operatorname{Germ}(-):$ SpatG $\rightarrow$ Gpoid is a (covariant) functor.

Proof. Let $\phi:\left(\Gamma_{1}, X_{1}\right) \rightarrow\left(\Gamma_{2}, X_{2}\right)$ be a spatial morphism. We first verify that the map $\operatorname{Germ}(\phi)$ is well-defined. Given $[\gamma, x] \in \operatorname{Germ}\left(\Gamma_{1}, X_{1}\right)$, suppose $\gamma^{\prime}, \gamma^{\prime \prime} \in \Gamma_{2}$ satisfy

$$
\phi \circ \gamma=\gamma^{\prime} \circ \phi=\gamma^{\prime \prime} \circ \phi .
$$

Then $\gamma^{\prime}$ and $\gamma^{\prime \prime}$ agree on $\phi\left(X_{1}\right)$, which is an open neighborhood of $\phi(x)$, hence we have $\left[\gamma^{\prime}, \phi(x)\right]=\left[\gamma^{\prime \prime}, \phi(x)\right]$. So the choice of $\gamma^{\prime}$ doesn't matter. As for the choice 
of $\gamma$, suppose $\tau \in \Gamma_{1}$ has the same germ over $x$ as $\gamma$, i.e. $\gamma_{\mid A}=\tau_{\mid A}$ for some open neighborhood $A$ of $x$ in $X_{1}$. Let $\tau^{\prime} \in \Gamma_{2}$ satisfy $\phi \circ \tau=\tau^{\prime} \circ \phi$. Then

$$
\gamma^{\prime} \circ \phi_{\mid A}=\phi \circ \gamma_{\mid A}=\phi \circ \tau_{\mid A}=\tau^{\prime} \circ \phi_{\mid A} \text {. }
$$

This means that $\gamma_{\mid \phi(A)}^{\prime}=\tau_{\mid \phi(A)}^{\prime}$, hence $\left[\gamma^{\prime}, \phi(x)\right]=\left[\tau^{\prime}, \phi(x)\right]$. So $\operatorname{Germ}(\phi)$ is welldefined.

Observe that the restriction to the unit spaces is just

$$
\operatorname{Germ}(\phi)^{(0)}=\phi: X_{1} \rightarrow X_{2} \text {. }
$$

From this we obtain

$$
s(\operatorname{Germ}(\phi)([\gamma, x]))=\phi(x)=\operatorname{Germ}(\phi)(s([\gamma, x])),
$$

and

$$
r(\operatorname{Germ}(\phi)([\gamma, x]))=\gamma^{\prime} \circ \phi(x)=\phi \circ \gamma(x)=\operatorname{Germ}(\phi)(r([\gamma, x])) .
$$

This means that $\operatorname{Germ}(\phi)$ takes composable pairs to composable pairs. As for preserving the product itself, we verify that

$$
\begin{aligned}
\operatorname{Germ}(\phi)([\tau, \gamma(x)]) \cdot \operatorname{Germ}(\phi)([\gamma, x]) & =\left[\tau^{\prime}, \phi \gamma(x)\right] \cdot\left[\gamma^{\prime}, \phi(x)\right]=\left[\tau^{\prime} \gamma^{\prime}, \phi(x)\right] \\
& =\operatorname{Germ}(\phi)([\tau \gamma, x]), \quad \text { since } \phi \tau \gamma=\phi \tau^{\prime} \gamma^{\prime}
\end{aligned}
$$

As $\operatorname{Germ}(\phi)^{(0)}=\phi$ is a local homeomorphism, it follows that $\operatorname{Germ}(\phi)$ is an étale homomorphism. Similar computations as above shows that Germ $(-)$ sends identity morphisms to identity morphisms and preserves composition of morphisms.

We record some consequences of this functoriality.

Corollary 5.5. Let $\phi:\left(X_{1}, \Gamma_{1}\right) \rightarrow\left(X_{2}, \Gamma_{2}\right)$ be a spatial morphism in SpatG.

(1) If $\phi$ is a spatial isomorphism, then $\operatorname{Germ}(\phi): \operatorname{Germ}\left(\Gamma_{1}, X_{1}\right) \rightarrow \operatorname{Germ}\left(\Gamma_{2}, X_{2}\right)$ is an isomorphism of étale groupoids.

(2) $\operatorname{Germ}(\phi)^{(0)}=\phi$, in particular $\operatorname{Germ}(\phi)$ maps $X_{1}$ onto $X_{2}$ if and only if $\phi$ is surjective.

(3) If $\phi: X_{1} \rightarrow X_{2}$ is injective, then $\operatorname{Germ}(\phi): \operatorname{Germ}\left(\Gamma_{1}, X_{1}\right) \rightarrow \operatorname{Germ}\left(\Gamma_{2}, X_{2}\right)$ is also injective.

(4) If $\phi: X_{1} \rightarrow X_{2}$ is surjective and $\phi \circ \Gamma_{1}=\Gamma_{2} \circ \phi$, then $\operatorname{Germ}(\phi): \operatorname{Germ}\left(\Gamma_{1}\right.$, $\left.X_{1}\right) \rightarrow \operatorname{Germ}\left(\Gamma_{2}, X_{2}\right)$ is also surjective.

Proof. Statement (1) follows immediately from functoriality, and statement (2) was observed in the proof of Proposition 5.4

(3) Assume that $\phi: X_{1} \rightarrow X_{2}$ is injective. Then clearly $\operatorname{Germ}(\phi)$ maps elements with distinct sources to distinct elements. So suppose

$$
\left[\gamma^{\prime}, \phi(x)\right]=\operatorname{Germ}(\phi)([\gamma, x])=\operatorname{Germ}(\phi)([\tau, x])=\left[\tau^{\prime}, \phi(x)\right] .
$$

Then $\gamma_{\mid A}^{\prime}=\tau_{\mid A}^{\prime}$ for some open neighborhood $A$ of $\phi(x)$ in $X_{2}$. As $\phi \circ \gamma=\gamma^{\prime} \circ \phi$ and $\phi \circ \tau=\tau^{\prime} \circ \phi$ we have that $\phi \circ \gamma$ and $\phi \circ \tau$ agree on $\phi^{-1}(A)$. The injectivity 
of $\phi$ now implies that $\gamma$ and $\tau$ agree on $\phi^{-1}(A)$, which is an open neighborhood of $x$, hence $[\gamma, x]=[\tau, x]$ and $\operatorname{Germ}(\phi)$ is injective.

(4) Suppose $\phi: X_{1} \rightarrow X_{2}$ is surjective and that $\phi \circ \Gamma_{1}=\Gamma_{2} \circ \phi$. Given $[\tau, y]$ in $\operatorname{Germ}\left(\Gamma_{2}, X_{2}\right)$, pick any $x \in X_{1}$ with $\phi(x)=y$. By assumption there is some $\gamma \in \Gamma_{1}$ such that $\phi \circ \gamma=\tau \circ \phi$, and then $\operatorname{Germ}(\phi)([\gamma, x])=[\tau, y]$.

Remark 5.6. It is natural to ask whether a spatial morphism $\phi$ from $\left(X_{1}, \Gamma_{1}\right)$ to $\left(X_{2}, \Gamma_{2}\right)$ induces a (algebraic) group homomorphism from $\Gamma_{1}$ to $\Gamma_{2}$. This is not so clear. But at least if $\phi: X_{1} \rightarrow X_{2}$ is injective and $\Gamma_{2}$ is locally closed, then one can define an injective group homomorphism $f_{\phi}: \Gamma_{1} \rightarrow \Gamma_{2}$ in the following way. First observe that given $\gamma \in \Gamma_{1}$, there is a $\gamma_{2} \in \Gamma_{2}$ with $\phi \circ \gamma=\gamma_{2} \circ \phi$, and then $\gamma_{2}\left(\phi\left(X_{1}\right)\right)=\phi\left(X_{1}\right)$ and $\operatorname{supp}\left(\left(\gamma_{2}\right)_{\mid \phi\left(X_{1}\right)}\right)=\phi(\operatorname{supp}(\gamma))$. Given another $\gamma_{3} \in \Gamma_{2}$ with $\phi \circ \gamma=\gamma_{3} \circ \phi$ we have

$$
\left(\gamma_{2}\right)_{\mid \phi\left(X_{1}\right)}=\left(\gamma_{3}\right)_{\mid \phi\left(X_{1}\right)} \in \operatorname{Homeo}_{c}\left(\phi\left(X_{1}\right)\right) .
$$

So we can define $f_{\phi}(\gamma)=\gamma^{\prime}$ to be the homeomorphism $\gamma^{\prime}$ on $X_{2}$ given by

$$
\left(\gamma^{\prime}\right)_{\mid \phi\left(X_{1}\right)}=\left(\gamma_{2}\right)_{\mid \phi\left(X_{1}\right)} \text { and } \quad\left(\gamma^{\prime}\right)_{\mid X_{2} \backslash \phi\left(X_{1}\right)}=\operatorname{id}_{X_{2} \backslash \phi\left(X_{1}\right)} .
$$

The homeomorphism $\gamma^{\prime}$ belongs to $\Gamma_{2}$ because $\Gamma_{2}$ is locally closed. It is straightforward to check that $f_{\phi}$ is an injective group homomorphism, and also that $\operatorname{supp}\left(f_{\phi}(\gamma)\right)=\phi(\operatorname{supp}(\gamma))$ for every $\gamma \in \Gamma_{1}$. If $\phi$ is a spatial isomorphism, then $f_{\phi}$ is a group isomorphism and $f_{\phi}$ satisfies $f_{\phi}(\gamma)=\phi \circ \gamma \circ \phi^{-1}$ for each $\gamma \in \Gamma_{1}$.

Remark 5.7. Viewing the functor Germ as a "free" functor turning a space-group pair into an effective ample Hausdorff groupoid (in the "most efficient" way), one could ask for a "forgetful" functor in the opposite direction. Proposition 4.6] suggests that this functor should be

$$
\llbracket-\rrbracket: \text { Gpoid } \rightarrow \text { SpatG } \quad \text { assigning } \mathcal{G} \mapsto\left(\llbracket \mathcal{G} \rrbracket, \mathcal{G}^{(0)}\right) .
$$

The natural choice for the morphisms is for an étale homomorphism $\Phi: \mathcal{G} \rightarrow \mathcal{H}$ to let

$$
\llbracket \Phi \rrbracket:=\Phi^{(0)}:\left(\llbracket \mathcal{G} \rrbracket, \mathcal{G}^{(0)}\right) \rightarrow\left(\llbracket \mathcal{H} \rrbracket, \mathcal{H}^{(0)}\right),
$$

i.e. restriction to the unit space. Unfortunately, this fails to be a morphism in SpatG in general. For injective étale homomorphisms though, the restriction to the unit spaces does yield an injective spatial morphism.

\section{Spatial Realization Theorems}

In this section, we shall study reconstruction of topological spaces from subgroups of their homeomorphism group in the sense of the following definition.

Definition 6.1. A class $K$ of space-group pairs is called faithful if every group isomorphism $\Phi: \Gamma_{1} \rightarrow \Gamma_{2}$, where $\left(\Gamma_{1}, X_{1}\right),\left(\Gamma_{2}, X_{2}\right) \in K$, is spatially implemented, 
that is, there is a homeomorphism $\phi: X_{1} \rightarrow X_{2}$ such that $\Phi(\gamma)=\phi \circ \gamma \circ \phi^{-1}$ for every $\gamma \in \Gamma_{1}$.

We stress the fact that the isomorphisms $\Phi$ considered in the preceding definition are, a priori, abstract group isomorphisms. They only "see" the algebraic structure of the $\Gamma_{i}$ 's, not the actions on the underlying spaces. We may rephrase faithfulness to saying that "every group isomorphism is a spatial isomorphism". In relation to the previous section, we obtain the following from Corollary 5.5 .

Proposition 6.2. Suppose $K$ is a faithful class of space-group pairs from SpatG. If $\left(\Gamma_{1}, X_{1}\right)$ and $\left(\Gamma_{2}, X_{2}\right)$ belong to $K$ and $\Gamma_{1}$ is isomorphic to $\Gamma_{2}$ as abstract groups, then the groupoids of germs $\operatorname{Germ}\left(\Gamma_{1}, X_{1}\right)$ and $\operatorname{Germ}\left(\Gamma_{2}, X_{2}\right)$ are isomorphic as topological groupoids.

In conjunction with Proposition 4.10 this will allow us to deduce that in many cases, the topological full group of an ample groupoid, considered as an abstract group, is a complete invariant for the isomorphism class of the groupoid. This will be done in the next section. The rest of this section will be devoted to proving two faithfulness results. The first one is a straightforward extension of Matui's spatial realization result [49, Theorem 3.5] to our locally compact setting (Theorem 6.6). This result will not only apply to the topological full group, but also to any subgroup containing the commutator. The second result we present (Theorem 6.19) has more relaxed assumptions on the "mixing properties" of the action, but we were not able to apply it to the commutator subgroup of the topological full group.

\subsection{The class $\mathrm{K}^{\mathrm{F}}$}

We now present the main definition from [49, Sec. 3], adapted to our setting.

Definition 6.3. We define the class $K^{F}$ to consist of all space-group pairs $(\Gamma, X)$ in SpatG which satisfy the following conditions:

(F1) For any $x \in X$ and any clopen neighborhood $A \subset X$ of $x$, there exists an involution $\alpha \in \Gamma$ such that $x \in \operatorname{supp}(\alpha)$ and $\operatorname{supp}(\alpha) \subseteq A$.

(F2) For any involution $\alpha \in \Gamma \backslash\{1\}$, and any non-empty clopen set $A \subseteq \operatorname{supp}(\alpha)$, there exists a $\beta \in \Gamma \backslash\{1\}$ such that $\operatorname{supp}(\beta) \subseteq A \cup \alpha(A)$ and $\alpha(x)=\beta(x)$ for every $x \in \operatorname{supp}(\beta)$.

(F3) For any non-empty clopen set $A \subseteq X$, there exists an $\alpha \in \Gamma$ such that $\operatorname{supp}(\alpha) \subseteq A$ and $\alpha^{2} \neq 1$.

Remark 6.4. In [49] Definition 3.1] there is also a condition (F0), stipulating that the support of any involution should be clopen. This is already implicit in the definition above, since all supports of elements in $\Gamma$ are assumed to be compact and open. We also remark that Definition 6.3 does not impose any countability restrictions on the space $X$. However, condition (F1) (and also (F3)) implies that $X$ cannot have isolated points. 
Remark 6.5. The notation $K^{F}$ to denote a class of space-group pairs is in the same style as Rubin uses in his paper 62. Elsewhere in the literature, in particular 27, 49], groups $\Gamma$ with $(\Gamma, X) \in K^{F}$ are called groups of class $F$ (and $X$ is assumed to be a (compact) Cantor space).

We now state a simple extension of Matui's Spatial Realization Theorem.

Theorem 6.6 (cf. [49, Theorem 3.5]). The class $K^{F}$ is faithful.

Proof. By closely inspecting the proof of [49, Theorem 3.5] and the three lemmas preceding it, one finds that the compactness of the spaces is not needed until the proof of [49, Theorem 3.5] itself. The lemmas preceding it are completely algebraic. Furthermore, the compactness is used only to guarantee that a certain intersection of supports become non-empty — by appealing to the finite intersection property. However, since all supports in our setting are already compact (by assumption) the conclusion that the intersection is non-empty still holds. The second countability is never needed. Therefore, Matui's proof remains valid.

Remark 6.7. We remark that Matui's proof of [49] Theorem 3.5] is similar to the approach used by Bezuglyi and Medynets in [3, Sec. 5], wherein the authors prove a precursor of Matui's Isomorphism Theorem for Cantor minimal systems. Both of these build on Fremlin's book [25, Sec. 384].

\subsection{The class $K^{\mathrm{LCC}}$}

We now turn to obtaining the second spatial realization result, by providing another faithful class of space group-pairs. In comparison with $K^{F}$, we'll impose more restrictions on the spaces (second countability — resulting in locally compact Cantor spaces), but the conditions on the actions will be less "localized" in some sense. We will of course still need the groups $\Gamma$ to be very "rich" in order to recover the action on the space $X$, but we do not focus solely on involutive group elements, as was the case for $K^{F}$.

Some of the (many) results from Rubins remarkable paper 62 will form the backbone of this spatial realization result. In that paper, Rubin exhibits the faithfulness of several general classes of space-group pairs. However, many of the classes considered there required quite different proofs. Arguably, the most commonly cited result from [62 in our context is [62, Corollary 3.5], but this spatial realization result is not strong enough to prove Theorem B], We essentially end up reproving Rubin's result on zero-dimensional spaces, but we obtain a slightly different statement. Also, our proof is a bit more straightforward (since we aim for a less general setting; namely perfect unit spaces of ample groupoids).

\subsubsection{Reconstructing the Boolean algebra $\mathcal{R}(X)$}

The main theorem from Sec. 2 of Rubin's paper (given below in Theorem 6.11) gives general conditions for when the abstract isomorphism class of 
a group $\Gamma \leq \operatorname{Homeo}(X)$ determines the Boolean algebra $\mathcal{R}(X)$, and the induced action by $\Gamma$ on it. We may view $\Gamma$ as a subgroup of $\operatorname{Aut}(\mathcal{R}(X))$ by taking images of regular open sets in $\mathcal{R}(X)$ under the homeomorphisms in $\Gamma$. In [62, Sec. 3], Rubin defines several classes of space-group pairs and proves, in a case-by-case manner, that the space $X$ and the action by $\Gamma$ on it, can be recovered from the induced action of $\Gamma$ on $\mathcal{R}(X)$. Let us begin with some terminology (adapted from [62]).

Definition 6.8. Let $(\Gamma, X)$ be a space-group pair.

(1) We say that $(\Gamma, X)$ is locally moving if for every non-empty open subset $A \subseteq X$ there exists $\gamma \in \Gamma \backslash\{1\}$ with $\operatorname{supp}(\gamma) \subseteq A$.

(2) An open set $B \subseteq X$ is called flexible if for every pair of open subsets $C_{1}, C_{2} \subseteq B$, if there exists $\gamma \in \Gamma$ such that $\gamma\left(C_{1}\right) \cap C_{2} \neq \emptyset$, then there exists $\tau \in \Gamma$ such that $\tau\left(C_{1}\right) \cap C_{2} \neq \emptyset$ and $\operatorname{supp}(\tau) \subseteq B$.

(3) We say that $(\Gamma, X)$ is locally flexible if every non-empty open subset $A$ contains a non-empty open flexible subset $B \subseteq A$.

Remark 6.9. Note that if $(\Gamma, X)$ is locally moving, then the space $X$ has no isolated points.

Remark 6.10. In 62], "locally moving" goes by the name "regionally disrigid", whilst the former terminology is from a later paper of Rubin [63].

We now state a special case of the main result from [62, Sec. 2].

Theorem 6.11 (cf. [62, Theorem 0.2, Theorem 2.14(a)]). Let $\left(\Gamma_{1}, X_{1}\right)$ and $\left(\Gamma_{2}, X_{2}\right)$ be in SpatG, and assume they are both locally moving and locally flexible. If $\Phi: \Gamma_{1} \rightarrow \Gamma_{2}$ is an isomorphism of groups, then there exists a Boolean isomorphism $\psi: \mathcal{R}\left(X_{1}\right) \rightarrow \mathcal{R}\left(X_{2}\right)$ such that $\psi(g(A))=\Phi(g)(\psi(A))$ for each $A \in \mathcal{R}\left(X_{1}\right)$ and $g \in \Gamma_{1}$.

If we think of $g$ and $\Phi(g)$ as elements in $\operatorname{Aut}\left(\mathcal{R}\left(X_{1}\right)\right)$ and $\operatorname{Aut}\left(\mathcal{R}\left(X_{2}\right)\right)$, respectively, then we can rewrite the conclusion in the preceding theorem as

$$
\Phi(g)=\psi \circ g \circ \psi^{-1} .
$$

Thus, Theorem 6.11 says that any group isomorphism between $\Gamma_{1}$ and $\Gamma_{2}$ is actually induced by an isomorphism of the Boolean algebras of regular open sets of the underlying spaces.

Remark 6.12. We remark that what Rubin proves in 62 Theorem 2.14(a)] is a somewhat stronger statement than the one we gave above. First of all, the spaces need really only be Hausdorff (and perfect). Rubin shows that if $(\Gamma, X)$ is locally moving and locally flexible, then starting with $\Gamma$ alone, one can canonically reconstruct the Boolean algebra $\mathcal{R}(X)$ (up to isomorphism) using only group theoretic constructions. Moreover, one obtains a natural action by $\Gamma$ on this Boolean algebra which is conjugate to the action by $\Gamma$ on $\mathcal{R}(X)$. The strategy of the proof is to model a regular set $A \in \mathcal{R}(X)$ by its rigid stabilizer $Q(A):=\{\gamma \in \Gamma \mid \operatorname{supp}(\gamma) \subseteq A\}$, and 
then to describe the Boolean operations in $\mathcal{R}(X)$ in group theoretic terms, in terms of the subgroups $Q(A)$. Finally, one shows that there are enough regular sets $A$ for which subgroups of the form $Q(A)$ can be detected inside $\Gamma$ in order to generate the whole of $\mathcal{R}(X)$.

\subsubsection{Reconstructing the space $X$}

We now turn to reconstructing $X$ (and the original action by $\Gamma$ ) from its Boolean algebra of regular sets. The strategy is to first impose conditions making it possible to detect clopenness. And then characterize the compact open sets among the clopen sets, which in turn allow us to recover $X$ from Stone duality.

Definition 6.13. Let $(\Gamma, X)$ be a space-group pair. A clopen set $A \subseteq X$ is said to be recognizable by $\Gamma$ if it satisfies:

(1) For every $\gamma \in \Gamma$ with $\gamma(A)=A$ the homeomorphism $\tau$ given by

$$
\tau(x)= \begin{cases}\gamma(x), & x \in A, \\ x, & \text { otherwise }\end{cases}
$$

belongs to $\Gamma$.

(2) For every $\gamma \in \Gamma$ with $\gamma(A) \cap A=\emptyset$ the involution $\alpha$ given by

$$
\alpha(x)= \begin{cases}\gamma(x), & x \in A, \\ \gamma^{-1}(x), & x \in \gamma(A), \\ x, & \text { otherwise, }\end{cases}
$$

belongs to $\Gamma$.

We shall see later that in our setting of topological full groups, all clopen subsets of the unit space are recognizable. And whenever this is the case, it is possible to characterize when a regular set is closed (i.e. clopen) using the following Boolean algebra notion.

Definition 6.14. Let $(\Gamma, X)$ be a space-group pair, and let $A \in \mathcal{R}(X)$ be a regular open set. We say that $A$ is weakly clopen if for every group element $\gamma \in \Gamma$ which satisfies $\gamma(A \cap \gamma(A))=A \cap \gamma(A)$, there exists an element $\rho \in \Gamma$ such that

(1) $\rho(B)=\gamma(B)$ for each $B \in \mathcal{R}(X)$ with $B \subseteq A \cap \gamma(A)$,

(2) $\rho(B)=B$ for each $B \in \mathcal{R}(X)$ with $B \subseteq \sim(A \cap \gamma(A))$.

Note that the notion of being weakly clopen is formulated solely in terms of the action by $\Gamma$ on the Boolean algebra $\mathcal{R}(X)$. And as the next result shows - under suitable hypotheses - being weakly clopen is the same as being clopen.

Lemma 6.15. Let $(\Gamma, X) \in$ SpatG. Assume that every clopen subset of $X$ is recognizable by $\Gamma$, and that the $\Gamma$-orbit of each point contains at least three points. 
Then a regular open set $A \in \mathcal{R}(X)$ is clopen if and only if both $A$ and $\sim A$ are weakly clopen.

Proof. This is a special case of [62, Lemma 3.45], where the dense subset $R$ is taken to be all of $\mathcal{R}(X)$. Assumptions 3.V.1(a)-(c) and 3.V.2(a), (b) preceding [62, Lemma 3.45] follow from those above. In particular, what Rubin calls "recognizably clopen" coincides with (2) in Definition 6.13 and "strongly recognizably clopen" is slightly weaker than (1) in Definition 6.13 (together with (2)).

In order to invoke Stone duality for Boolean spaces we need to recover the generalized Boolean algebra of compact open sets. The previous lemma gives us the clopen sets, and from these we obtain the compact open ones as follows.

Lemma 6.16. Let $X$ be a second countable Boolean space. Then $X$ is compact if and only if $\mathrm{CO}(X)$ is countable.

Proof. If $X$ is compact, then $\mathrm{CO}(X)=\mathrm{CK}(X)$, and any second countable space has countably many compact open subsets.

Suppose $X$ is non-compact. Let $\left\{K_{n}\right\}_{n=1}^{\infty}$ be a countable basis for $X$ consisting of compact open sets. Now form the compact open sets $C_{k}=\bigcup_{n=1}^{k} K_{n}$. As $X$ is not compact, we must have $C_{k} \neq X$ for each $k$. Also, $C_{k} \subseteq C_{k+1}$ and they cover $X$. By passing to a subsequence, if necessary, we may assume that $C_{k} \subsetneq C_{k+1}$ for each $k$. Finally, let $D_{k}=C_{k+1} \backslash C_{k}$. Then the $D_{k}$ 's are pairwise disjoint non-empty compact open sets. We claim that for each subset $S$ of the natural numbers, the set $\bigcup_{k \in S} D_{k}$ is clopen. And then we have produced uncountably many distinct clopen sets. The claim follows from the fact that for each $C_{m}$, the intersection $C_{m} \cap\left(\bigcup_{k \in S} D_{k}\right)$ is a finite intersection, hence closed, and that the $C_{m}$ 's cover $X$.

Corollary 6.17. Let $X$ be a second countable Boolean space, and let $A \in \mathrm{CO}(X)$ be a clopen set. Then $A$ is compact if and only if the set $\{B \in \mathrm{CO}(X) \mid B \subseteq A\}$ is countable.

Proof. The set $\{B \in \mathrm{CO}(X) \mid B \subseteq A\}$ coincides with $\mathrm{CO}(A)$ when viewing $A$ as a subspace of $X$. The result now follows from Lemma 6.16

This shows that in the generalized Boolean algebra $\mathrm{CO}(X)$ compactness is characterized by having only countably many elements below. We are now ready to define the class $K^{\mathrm{LCC}}$ and give the second spatial realization result of this section.

Definition 6.18. We define the class $K^{\mathrm{LCC}}$ to consist of all space-group pairs $(\Gamma, X)$ in SpatG which satisfy the following conditions:

(K1) $X$ is a locally compact Cantor space.

(K2) $(\Gamma, X)$ is locally moving.

(K3) $(\Gamma, X)$ is locally flexible. 
(K4) Every clopen subset of $X$ is recognizable by $\Gamma$.

(K5) The $\Gamma$-orbit of each point contains at least three points.

Theorem 6.19 (cf. [62, Theorem 3.50(a)]). The class $K^{\mathrm{LCC}}$ is faithful.

Proof. Suppose we have two space-group pairs $\left(\Gamma_{1}, X_{1}\right),\left(\Gamma_{2}, X_{2}\right) \in K^{\mathrm{LCC}}$ and a group isomorphism $\Phi: \Gamma_{1} \rightarrow \Gamma_{2}$. Invoking Theorem 6.11 yields an isomorphism of Boolean algebras $\psi: \mathcal{R}\left(X_{1}\right) \rightarrow \mathcal{R}\left(X_{2}\right)$ such that $\psi(g(A))=\Phi(g)(\psi(U))$ for each $A \in \mathcal{R}\left(X_{1}\right)$ and $g \in \Gamma_{1}$. We first argue that $\psi\left(\operatorname{CO}\left(X_{1}\right)\right)=\operatorname{CO}\left(X_{2}\right)$, and then that $\psi\left(\mathrm{CK}\left(X_{1}\right)\right)=\mathrm{CK}\left(X_{2}\right)$.

First of all, note that both $\mathrm{CO}\left(X_{i}\right)$ and $\mathrm{CK}\left(X_{i}\right)$ are invariant under $\Gamma_{i}(i=1,2)$. Lemma 6.15 characterizes clopenness of regular sets in $X_{i}$ solely in terms of the (induced) actions by $\Gamma_{i}$ on $\mathcal{R}\left(X_{i}\right)$. Since $\psi$ is an equivariant Boolean algebra isomorphism, it follows that $\psi\left(\mathrm{CO}\left(X_{1}\right)\right)=\mathrm{CO}\left(X_{2}\right)$. Next, Corollary 6.17 characterizes compactness of a clopen set in terms of a countability condition in the generalized Boolean algebra $\mathrm{CO}\left(X_{i}\right)$. Clearly, this is then also preserved by $\psi$. Consequently, $\psi$ restricts to an equivariant isomorphism of the generalized Boolean algebras $\operatorname{CK}\left(X_{1}\right)$ and $\operatorname{CK}\left(X_{2}\right)$.

By applying the Stone functor to the generalized Boolean algebra isomorphism

$$
\psi: \mathrm{CK}\left(X_{1}\right) \rightarrow \mathrm{CK}\left(X_{2}\right)
$$

we obtain a homeomorphism

$$
\mathbb{S}(\psi): \mathbb{S}\left(\mathrm{CK}\left(X_{2}\right)\right) \rightarrow \mathbb{S}\left(\mathrm{CK}\left(X_{1}\right)\right)
$$

of the spaces of ultrafilters. The induced actions by the groups $\Gamma_{i}$ on $\mathbb{S}\left(\mathrm{CK}\left(X_{i}\right)\right)$ is given by $g \cdot \alpha=\{g(K) \mid K \in \alpha\}$ for an ultrafilter $\alpha \in \mathbb{S}\left(\mathrm{CK}\left(X_{i}\right)\right)$. Finally, let $\phi: X_{1} \rightarrow X_{2}$ be the homeomorphism given by the composition

$$
X_{1} \stackrel{\Omega_{X_{1}}}{\longrightarrow} \mathbb{S}\left(\mathrm{CK}\left(X_{1}\right)\right) \stackrel{\mathbb{S}(\psi)^{-1}}{\longrightarrow} \mathbb{S}\left(\mathrm{CK}\left(X_{2}\right)\right) \stackrel{\Omega_{X_{2}}^{-1}}{\longrightarrow} X_{2},
$$

where $\Omega_{X_{i}}$ is the canonical homeomorphism mapping a point to its compact open neighborhood ultrafilter. It is now easy to check that the original group isomorphism $\Phi$ is spatially implemented by $\phi$, i.e. that $\Phi(g)=\phi \circ g \circ \phi^{-1}$ for each $g \in \Gamma_{1}$. This completes the proof.

Remark 6.20. As mentioned in Sec. 1 Medynets has obtained a spatial realization result for full groups of group actions on the Cantor space [52. The arguments therein also apply to the topological full group, and could be adapted to the topological full group of the ample groupoids over locally compact Cantor spaces considered here. And then in turned be used to prove Theorem [B instead of using Theorem 6.19. Medynets' starting point is a Boolean algebra reconstruction result of Fremlin [25, Theorem 384D]. This result is very similar to Rubin's Boolean algebra reconstruction result; Theorem 6.11, Rubin requires the space-group pair to be locally moving and locally flexible, whereas Fremlin requires it to be locally moving 
in terms of involutions. Yet they both apply to the topological full group, since it is both (globally) flexible and has enough involutions to witness locally moving. Medynets then goes on to characterize the clopen sets among the regular open sets in an algebraic way and use this to show that the Boolean algebra isomorphism must preserve the Boolean subalgebra of clopen subsets and in turn give rise to a spatial isomorphism via Stone duality. This is exactly the same approach as we use here, via Rubin, but Medynets' characterization of the clopens [52. Lemma 2.5] looks (at least on the surface) a bit different from the one we give here in Lemma6.15. Finally, we remark that Medynets' arguments does not seem to apply to the commutator subgroup either (cf. Remark 7.11).

\section{Isomorphism Theorems for Ample Groupoids}

In this section, we shall apply the spatial realization results of the previous section to (subgroups of) the topological full group. As corollaries we are able to reconstruct certain ample groupoids from their topological full group. The two faithful classes considered in the previous section allows us to lift an abstract group isomorphism of (subgroups of) the topological full groups to a spatial one. This in turn yields an isomorphism of the associated groupoids of germs (cf. Corollary 5.5). In order to conclude that the groupoids themselves are isomorphic we need, by Proposition 4.10 and Corollary 4.13 to assume that the subgroups in question cover the groupoids. As we saw in Lemma 4.9 if every $\mathcal{G}$-orbit has length at least two, or respectively, three, then $\llbracket \mathcal{G} \rrbracket$, or respectively, any $\Gamma$ with $D(\llbracket \mathcal{G} \rrbracket) \leq \Gamma \leq \llbracket \mathcal{G} \rrbracket$, covers $\mathcal{G}$.

We first extract an isomorphism theorem from the faithfulness of the class $K^{F}$. For a general ample groupoid the only general condition, we know to imply that $\left(\llbracket \mathcal{G} \rrbracket, \mathcal{G}^{(0)}\right)$ belong to $K^{F}$ is minimality. So for general groupoids, we obtain only a straightforward minor extension of [49] Theorem 3.9 and 3.10] in Theorem 7.2 below. However, for the class of graph groupoids we will see in Sec. 10 that we can weaken minimality quite a lot and still have the topological full group (and its commutator) in $K^{F}$, and thereby obtain a significantly more general result within the class of graph groupoids. It would therefore be interesting to find general conditions on a general ample groupoid $\mathcal{G}$, weaker than minimality, ensuring that $\left(\llbracket \mathcal{G} \rrbracket, \mathcal{G}^{(0)}\right)$ and $\left(\mathrm{D}(\llbracket \mathcal{G} \rrbracket), \mathcal{G}^{(0)}\right)$ belong to $K^{F}$.

Proposition 7.1 (cf. [49, Proposition 3.6]). Let $\mathcal{G}$ be an effective ample Hausdorff groupoid whose unit space has no isolated points. If $\mathcal{G}$ is minimal and $\Gamma$ is any subgroup of $\llbracket \mathcal{G} \rrbracket$ containing $\mathrm{D}(\llbracket \mathcal{G} \rrbracket)$, then $\left(\Gamma, \mathcal{G}^{(0)}\right) \in K^{F}$.

Proof. The proof of [49, Proposition 3.6] goes through verbatim in this slightly more general setting. The proof makes heavy use of the minimality of $\mathcal{G}$ and combine this with Lemma 3.8 to find the desired elements in $\mathrm{D}(\llbracket \mathcal{G} \rrbracket)$.

Theorem 7.2. Let $\mathcal{G}_{1}, \mathcal{G}_{2}$ be effective ample minimal Hausdorff groupoids whose unit spaces have no isolated points. Suppose $\Gamma_{1}, \Gamma_{2}$ are subgroups such that 
$\mathrm{D}\left(\llbracket \mathcal{G}_{i} \rrbracket\right) \leq \Gamma_{i} \leq \llbracket \mathcal{G}_{i} \rrbracket$. If $\Gamma_{1} \cong \Gamma_{2}$ as abstract groups, then $\mathcal{G}_{1} \cong \mathcal{G}_{2}$ as topological groupoids. In particular, the following are equivalent:

(1) $\mathcal{G}_{1} \cong \mathcal{G}_{2}$ as topological groupoids.

(2) $\llbracket \mathcal{G}_{1} \rrbracket \cong \llbracket \mathcal{G}_{2} \rrbracket$ as abstract groups.

(3) $\mathrm{D}\left(\llbracket \mathcal{G}_{1} \rrbracket\right) \cong \mathrm{D}\left(\llbracket \mathcal{G}_{2} \rrbracket\right)$ as abstract groups.

Proof. Clearly every $\mathcal{G}_{i}$-orbit is infinite, for $i=1,2$. Thus the result follows from combining Proposition 7.1, Theorem 6.6. Proposition 6.2 Lemma 4.9 and Proposition 4.10 .

Remark 7.3. For transformation groupoids arising from minimal $\mathbb{Z}$-actions on locally compact Cantor spaces, a variant of this result appears in [46, Theorem 4.13(vi)]. See also Remark 3.3

Remark 7.4. In [49 Theorem 3.10] the kernel of the so-called index map also appears (as $\llbracket \mathcal{G} \rrbracket_{0}$ ). We could equally well have included it in Theorem 7.2 since it is a distinguished subgroup lying between $\llbracket \mathcal{G} \rrbracket$ and $\mathrm{D}(\llbracket \mathcal{G} \rrbracket)$.

Our next goal is to analyze the conditions in the definition of the class $K^{\mathrm{LCC}}$, when the space-group pair under consideration is the topological full group and the unit space of an ample groupoid. Unfortunately, the commutator subgroup $\mathrm{D}(\llbracket \mathcal{G} \rrbracket)$ does not seem to belong to $K^{\mathrm{LCC}}$, which is why we only consider $\llbracket \mathcal{G} \rrbracket$ itself (see Remark 7.11 below). We begin by showing that the groupoid-orbits coincide with the orbits of the action by the topological full group on the unit space.

Lemma 7.5. Let $\mathcal{G}$ be an effective ample groupoid and let $x \in \mathcal{G}^{(0)}$. Then

$$
\operatorname{Orb}_{\mathcal{G}}(x)=\operatorname{Orb}_{\llbracket \mathcal{G} \rrbracket \curvearrowright \mathcal{G}^{(0)}}(x) .
$$

Proof. The inclusion $\operatorname{Orb}_{\llbracket \mathcal{G} \rrbracket \curvearrowright \mathcal{G}^{(0)}}(x) \subseteq \operatorname{Orb}_{\mathcal{G}}(x)$ is immediate from the definition of the topological full group. For the reverse inclusion, suppose $y \in \operatorname{Orb}_{\mathcal{G}}(x)$ is distinct from $x$, and let $\gamma \in \mathcal{G}$ be an arrow from $x$ to $y$. Applying Lemma 3.9 to $\gamma$ we obtain an element $\pi_{U} \in \llbracket \mathcal{G} \rrbracket$ with $\pi_{U}(x)=y$. Thus $y \in \operatorname{Orb}_{\llbracket \mathcal{G} \rrbracket \curvearrowright \mathcal{G}^{(0)}}(x)$.

In other words, when the space group pair is $\left(\llbracket \mathcal{G} \rrbracket, \mathcal{G}^{(0)}\right)$ condition $(\mathrm{K} 5)$ of Definition 6.18 is equivalent to saying that every $\mathcal{G}$-orbit has length at least three (which, incidentally, implies that $\llbracket \mathcal{G} \rrbracket$ covers $\mathcal{G}$ ). Next, we show that conditions (K3) and (K4) of Definition 6.18 are always satisfied for topological full groups. In fact, $\left(\llbracket \mathcal{G} \rrbracket, \mathcal{G}^{(0)}\right)$ is even "globally flexible".

Lemma 7.6. Let $\mathcal{G}$ be an effective ample groupoid. Then every open subset of $\mathcal{G}^{(0)}$ is flexible with respect to $\llbracket \mathcal{G} \rrbracket$. In particular, $\left(\llbracket \mathcal{G} \rrbracket, \mathcal{G}^{(0)}\right)$ is locally flexible.

Proof. Let $A$ be a non-empty open subset of $\mathcal{G}^{(0)}$, and let $B_{1}, B_{2}$ be two open subsets of $A$. We may assume that these are disjoint, for otherwise the identity 
homeomorphism trivially witnesses flexibility. Suppose $\pi_{U} \in \llbracket \mathcal{G} \rrbracket$ satisfies $\pi_{U}\left(B_{1}\right) \cap B_{2} \neq \emptyset$. Then there is a $g \in U$ with $s(g) \in B_{1}$ and $r(g) \in B_{2}$. Lemma 3.9 applied to $g$ and $B_{1} \sqcup B_{2}$ produces an element $\pi_{V} \in \llbracket \mathcal{G} \rrbracket$ satisfying $\operatorname{supp}\left(\pi_{V}\right) \subseteq B_{1} \sqcup B_{2} \subseteq A$ and $\pi_{V}\left(B_{1}\right) \cap B_{2} \neq \emptyset$. Hence $A$ is flexible.

Lemma 7.7. Let $\mathcal{G}$ be an effective ample groupoid. Then every clopen subset of $\mathcal{G}^{(0)}$ is recognizable by $\llbracket \mathcal{G} \rrbracket$.

Proof. Let $A \subseteq \mathcal{G}^{(0)}$ be clopen.

(1) Suppose $\pi_{U} \in \llbracket \mathcal{G} \rrbracket$ satisfies $\pi_{U}(A)=A$. Then $V=s_{\mid U}^{-1}(A) \subseteq U$ is a clopen bisection with $s(V)=r(V)=A$. Then $\tilde{V}$ as in Lemma [3.7 is a full bisection with $\operatorname{supp}\left(\pi_{\tilde{V}}\right) \subseteq \operatorname{supp}\left(\pi_{U}\right)$, hence $\pi_{\tilde{V}} \in \llbracket \mathcal{G} \rrbracket$. The homeomorphism $\pi_{\tilde{V}}$ is the one from condition (1) of Definition 6.13

(2) Suppose now that $\pi_{U} \in \llbracket \mathcal{G} \rrbracket$ satisfies $\pi_{U}(A) \cap A=\emptyset$. Once more we set $V=s_{\mid U}^{-1}(A)$. Then $s(V) \cap r(V)=A \cap \pi_{U}(A)=\emptyset$. The full bisection $\hat{V}$ as in Lemma 3.8 also has compact support $\operatorname{since} \operatorname{supp}\left(\pi_{\hat{V}}\right) \subseteq \operatorname{supp}\left(\pi_{U}\right)$, and so $\pi_{\hat{V}} \in \llbracket \mathcal{G} \rrbracket$. The involution $\pi_{\hat{V}}$ is the one from condition (2) of Definition 6.13.

It remains to consider condition (K2) of Definition 6.18. Inspired by [52, Proposition 2.2], we introduce the notion of a non-wandering groupoid, in order to characterize when $\left(\llbracket \mathcal{G} \rrbracket, \mathcal{G}^{(0)}\right)$ is locally moving in terms of the groupoid $\mathcal{G}$.

Definition 7.8. Let $\mathcal{G}$ be an ample groupoid. A subset $A \subseteq \mathcal{G}^{(0)}$ is called wandering if $\left|A \cap \operatorname{Orb}_{\mathcal{G}}(x)\right|=1$ for all $x \in A$. We say that $\mathcal{G}$ is non-wandering if $\mathcal{G}^{(0)}$ has no non-empty clopen wandering subsets.

In words, a non-wandering groupoid is one in which every clopen subset of the unit space meets some orbit at least twice. This may be viewed as a "mixing condition" which is far weaker than minimality. For if $\mathcal{G}$ is minimal, then in particular the set $A \cap \operatorname{Orb}_{\mathcal{G}}(x)$ is infinite (being dense) for each clopen neighborhood $A$ of $x$.

Proposition 7.9. Let $\mathcal{G}$ be an effective ample Hausdorff groupoid. Then the following are equivalent:

(1) The space-group pair $\left(\llbracket \mathcal{G} \rrbracket, \mathcal{G}^{(0)}\right)$ is locally moving.

(2) The groupoid $\mathcal{G}$ is non-wandering.

Proof. Let $A$ be a non-empty clopen subset of $\mathcal{G}^{(0)}$. We will prove that $A$ meets some $\mathcal{G}$-orbit twice (i.e. $A$ is not wandering) if and only if there is some element $\pi_{U} \in \llbracket \mathcal{G} \rrbracket \backslash\{1\}$ with $\operatorname{supp}\left(\pi_{U}\right) \subseteq A$. If $\emptyset \neq \operatorname{supp}\left(\pi_{U}\right) \subseteq A$, then, since both sets are clopen, there is an $x \in A$ with $x \neq \pi_{U}(x) \in A$. In other words, $\left|A \cap \operatorname{Orb}_{\mathcal{G}}(x)\right| \geq 2$. Conversely, if $\left|A \cap \operatorname{Orb}_{\mathcal{G}}(x)\right| \geq 2$ holds for some $x \in A$, then there is a $g \in \mathcal{G} \backslash \mathcal{G}^{\prime}$ such that $s(g)$ and $r(g)$ both belong to $A$. Now Lemma 3.9 gives us a nontrivial 
group element in $\llbracket \mathcal{G} \rrbracket$ supported on $A$. As the clopens form a base for the topology on $\mathcal{G}^{(0)}$ we are done.

Putting it all together, we arrive at the second main result of this section.

Theorem 7.10. Let $\mathcal{G}_{1}, \mathcal{G}_{2}$ be effective ample Hausdorff groupoids over locally compact Cantor spaces. Suppose that, for $i=1,2, \mathcal{G}_{i}$ is non-wandering and that each $\mathcal{G}_{i}$-orbit has length at least three. Then any isomorphism between $\llbracket \mathcal{G}_{1} \rrbracket$ and $\llbracket \mathcal{G}_{2} \rrbracket i s$ spatial. In particular, the following are equivalent:

(1) $\mathcal{G}_{1} \cong \mathcal{G}_{2}$ as topological groupoids.

(2) $\llbracket \mathcal{G}_{1} \rrbracket \cong \llbracket \mathcal{G}_{2} \rrbracket$ as abstract groups.

Remark 7.11. It would be desirable to also obtain a spatial realization result for the commutator subgroup $\mathrm{D}(\llbracket \mathcal{G} \rrbracket)$ in terms of the class $K^{\mathrm{LCC}}$. Unfortunately, we were not able to show that $\mathrm{D}(\llbracket \mathcal{G} \rrbracket)$ satisfies condition $(\mathrm{K} 4)$. This is also the reason why the arguments of 52] do not apply to the commutator subgroup either. However, it might be that Theorem 7.10 holds for the commutator subgroups as well.

As mentioned above, non-wandering is a much weaker "mixing property" than minimality. Below we include two other "mixing properties" that lie between nonwandering and minimality.

Definition 7.12 (cf. [54, p. 8]). An ample groupoid $\mathcal{G}$ is called locally minimal if there exists a basis for $\mathcal{G}^{(0)}$ consisting of clopen sets $A$ such that $\mathcal{G}_{\mid A}$ is minimal.

Definition 7.13. An ample groupoid $\mathcal{G}$ is called densely minimal if for every nonempty open subset $A$ of $\mathcal{G}^{(0)}$ there exists a non-empty clopen subset $B \subseteq A$ such that $\mathcal{G}_{\mid B}$ is minimal.

We clearly have the following implications for an ample groupoid:

$$
\text { minimal } \Rightarrow \text { locally minimal } \Rightarrow \text { densely minimal } \Rightarrow \text { non-wandering. }
$$

We will give examples of densely minimal groupoids which are not minimal in the next section (Examples 9.6 and 9.7), as well as non-wandering groupoids which are not densely minimal (Remark 10.8).

\section{Graph Groupoids}

The rest of the paper will be focused on graph groupoids. This section recalls the relevant terminology for graphs and their associated groupoids (as they appear in the literature on graph algebras). We also record the characterizations of many properties of a graph groupoid in terms of the graph. This is fairly standard and may also be found in many other papers, e.g. [10, 36]. 


\subsection{Graph terminology}

By a graph we shall always mean a directed graph, which is a quadruple $E=\left(E^{0}, E^{1}, r, s\right)$, where $E^{0}, E^{1}$ are (non-empty) sets and $r, s: E^{1} \rightarrow E^{0}$ are maps. The elements in $E^{0}$ and $E^{1}$ are called vertices and edges, respectively, while the maps $r$ and $s$ are called the range $e^{\mathrm{e}}$ and source map, respectively. We say that $E$ is finite if $E^{0}$ and $E^{1}$ both are finite sets, and similarly that $E$ is countable if $E^{0}$ and $E^{1}$ are countable.

A path in a graph $E$ is a sequence of edges $\mu=e_{1} e_{2} \ldots e_{n}$ which satisfies $r\left(e_{i}\right)=s\left(e_{i+1}\right)$ for $1 \leq i \leq n-1$. The length of $\mu$ is $|\mu|:=n$. The set of paths of length $n$ is denoted $E^{n}$. The vertices, $E^{0}$, are considered trivial paths of length zero. The set of all finite paths is $E^{*}:=\bigcup_{n=0}^{\infty} E^{n}$. The range and source maps extend naturally to $E^{*}$ by setting $r(\mu):=r\left(e_{n}\right)$ and $s(\mu):=s\left(e_{1}\right)$. For $v \in E^{0}$, we set $s(v)=r(v)=v$. Given another path $\lambda=f_{1} \ldots f_{m}$ with $s(\lambda)=r(\mu)$ we denote the concatenated path $e_{1} \ldots e_{n} f_{1} \ldots f_{m}$ by $\mu \lambda$. In particular, we set $s(\mu) \mu=\mu=$ $\mu r(\mu)$ for each $\mu \in E^{*}$. Given two paths $\mu, \mu^{\prime} \in E^{*}$ we write $\mu<\mu^{\prime}$ if there exists a path $\lambda$ with $|\lambda| \geq 1$ such that $\mu^{\prime}=\mu \lambda$. Writing $\mu \leq \mu^{\prime}$ allows for $\mu=\mu^{\prime}$. We say that $\mu$ and $\mu^{\prime}$ are disjoint if $\mu \not \leq \mu^{\prime}$ and $\mu^{\prime} \not \leq \mu$, i.e. neither is a subpath of the other.

A cycle is a nontrivial path $\mu$ (i.e. $|\mu| \geq 1$ ) with $r(\mu)=s(\mu)$, and we say that $\mu$ is based at $s(\mu)$. We also say that the vertex $s(\mu)$ supports the cycle $\mu$. By a loop we mean a cycle of length one. Beware that some authors use the term loop to denote what we here call cycles. When $\mu$ is a cycle and $k \in \mathbb{N}, \mu^{k}$ denotes the cycle $\mu \mu \ldots \mu$, where $\mu$ is repeated $k$ times. A cycle $\mu=e_{1} \ldots e_{n}$ is called a return path if $r\left(e_{i}\right) \neq r(\mu)$ for all $i<n$. This simply means that $\mu$ does not pass through $s(\mu)$ multiple times. An exit for a path $\mu=e_{1} \ldots e_{n}$ is an edge $e$ such that $s(e)=s\left(e_{i}\right)$ and $e \neq e_{i}$ for some $1 \leq i \leq n$.

For $v, w \in E^{0}$ we set $v E^{n}:=\left\{\mu \in E^{n} \mid s(\mu)=v\right\}, E^{n} w:=\left\{\mu \in E^{n} \mid r(\mu)=w\right\}$ and $v E^{n} w:=v E^{n} \cap E^{n} w$. A vertex $v \in E^{0}$ is called a sink if $v E^{1}=\emptyset$, and a source if $E^{1} v=\emptyset$. Further, $v$ is called an infinite emitter if $v E^{1}$ is an infinite set. The set of regular vertices is $E_{\mathrm{reg}}^{0}:=\left\{v \in E^{0}|0<| v E^{1} \mid<\infty\right\}$, and the set of singular vertices is $E_{\text {sing }}^{0}:=E^{0} \backslash E_{\text {reg }}^{0}$. In other words, sinks and infinite emitters are singular vertices, while all other vertices are regular. We equip the vertex set $E^{0}$ with a preorder $\geq$ by defining $v \geq w$ if and only if $v E^{*} w \neq \emptyset$, i.e. there is a path from $v$ to $w$. The graph $E$ is called strongly connected if for each pair of vertices $v, w \in E^{0}$ we have $v \geq w$.

To close this subsection, we describe three exit conditions on graphs that appear frequently in the graph algebra literature. They will play a central role in what follows. A graph $E$ is said to satisfy Condition $(L)$ if every cycle in $E$ has an exit. The graph $E$ satisfies Condition $(K)$ if for every vertex $v \in E^{0}$, either there is

\footnotetext{
${ }^{\mathrm{e}}$ Although the notation collides with the range and source maps in a groupoid, both conventions are well established. In the sequel it will always be clear from context whether we mean the source/range of an edge in a graph or of an element in a groupoid.
} 
no return path based at $v$ or there are at least two distinct return paths based at $v$. We say that $E$ satisfies Condition $(I)$ if for every vertex $v \in E^{0}$, there exists a vertex $w \in E^{0}$ supporting at least two distinct return paths and $v \geq w$. These conditions first appeared in [17, 35, 36], respectively. In general, Condition $(K)$ and $(I)$ both imply $(L)$, while $(K)$ and $(I)$ are not comparable. For graphs with finitely many vertices and no sinks, Condition $(I)$ is equivalent to Condition $(L)$.

\subsection{The boundary path space}

An infinite path in a graph $E$ is an infinite sequence of edges $x=e_{1} e_{2} e_{3} \ldots$ such that $r\left(e_{i}\right)=s\left(e_{i+1}\right)$ for all $i \in \mathbb{N}$. We define $s(x):=s\left(e_{1}\right)$ and $|x|:=\infty$. The set of all infinite paths in $E$ is denoted $E^{\infty}$. Given a finite path $\mu=f_{1} \ldots f_{n}$ and an infinite path $x=e_{1} e_{2} e_{3} \ldots \in E^{\infty}$ such that $r(\mu)=s(x)$ we denote the infinite path $f_{1} \ldots f_{n} e_{1} e_{2} e_{3} \ldots$ by $\mu x$. For natural numbers $m<n$, we set $x_{[m, n]}:=e_{m} e_{m+1} \ldots e_{n}$, and we denote the infinite path $e_{m} e_{m+1} e_{m+2} \ldots$ by $x_{[m, \infty)}$. Given a cycle $\lambda \in E^{*}$ we denote the infinite path $\lambda \lambda \lambda \ldots$ by $\lambda^{\infty}$. An infinite path of the form $\mu \lambda^{\infty}$, where $\lambda$ is a cycle with $s(\lambda)=r(\mu)$, is called eventually periodic. An infinite path $e_{1} e_{2} \ldots \in E^{\infty}$ is wandering if the set $\left\{i \in \mathbb{N} \mid s\left(e_{i}\right)=v\right\}$ is finite for each $v \in E^{0}$. Note that there are no wandering infinite paths in a graph with finitely many vertices. We call a wandering infinite path $e_{1} e_{2} \ldots \in E^{\infty}$ a semi-tail s $^{\mathrm{f}}$ if $s\left(e_{i}\right) E^{1}=\left\{e_{i}\right\}$ for each $i \in \mathbb{N}$. The graph $E$ is called cofinal if for every vertex $v \in E^{0}$ and for every infinite path $e_{1} e_{2} \ldots \in E^{\infty}$, there exists $n \in \mathbb{N}$ such that $v \geq s\left(e_{n}\right)$.

The boundary path space of $E$ is

$$
\partial E:=E^{\infty} \cup\left\{\mu \in E^{*} \mid r(\mu) \in E_{\text {sing }}^{0}\right\},
$$

whose topology will be specified shortly. Note that if $v \in E^{0}$ is a singular vertex, then $v$ belongs to $\partial E$. For any vertex $v \in E^{0}$ we define $v \partial E:=\{x \in \partial E \mid s(x)=v\}$ and similarly $v E^{\infty}:=\left\{x \in E^{\infty} \mid s(x)=v\right\}$. The cylinder set of a finite path $\mu \in E^{*}$ is $Z(\mu):=\{\mu x \mid x \in r(\mu) \partial E\}$. Given a finite subset $F \subseteq r(\mu) E^{1}$, we define the "punctured" cylinder set $Z(\mu \backslash F):=Z(\mu) \backslash\left(\bigcup_{e \in F} Z(\mu e)\right)$. Note that two finite paths are disjoint if and only if their cylinder sets are disjoint sets. A basis for the topology on the boundary path space $\partial E$ is given by

$$
\left\{Z(\mu \backslash F) \mid \mu \in E^{*}, F \subseteq \text { finite } r(\mu) E^{1}\right\},
$$

cf. 69. Each basic set $Z(\mu \backslash F)$ is compact open and these separate points, so $\partial E$ is a Boolean space. Moreover, each open set in $\partial E$ is a disjoint union of basic sets $Z(\mu \backslash F)$ ([10, Lemma 2.1]). The boundary path space $\partial E$ is second countable exactly when $E$ is countable, and it is compact if and only if $E^{0}$ is finite. When it comes to (topologically) isolated points, these are classified as follows.

${ }^{\mathrm{f}}$ By comparison, a tail is a wandering path with $s\left(e_{i}\right) E^{1}=\left\{e_{i}\right\}=E^{1} r\left(e_{i}\right)$ for all $i$, cf. [2]. 
Proposition 8.1 ([16, Proposition 3.1]). Let $E$ be a graph.

(1) If $v \in E^{0}$ is a sink, then any finite path $\mu \in E^{*}$ with $r(\mu)=v$ is an isolated point in $\partial E$.

(2) If $x=\mu \lambda^{\infty} \in E^{\infty}$ is eventually periodic, then $x$ is an isolated point if and only if the cycle $\lambda$ has no exit.

(3) If $x=e_{1} e_{2} \ldots \in E^{\infty}$ is wandering, then $x$ is an isolated point if and only if for some $n \in \mathbb{N}, e_{n} e_{n+1} \ldots$ is a semi-tail.

These are the only isolated points in $\partial E$.

We define $\partial E^{\geq n}:=\{x \in \partial E|| x \mid \geq n\}$ and $\partial E^{n}:=\{x \in \partial E|| x \mid=n\}$ for $n$ a natural number. Each of the sets $\partial E^{\geq n}$ is an open subset of $\partial E$. The shift map on $E$ is the map $\sigma_{E}: \partial E^{\geq 1} \rightarrow \partial E$ given by $\sigma_{E}\left(e_{1} e_{2} e_{3} \ldots\right)=e_{2} e_{3} e_{4} \ldots$ for $e_{1} e_{2} e_{3} \ldots \in \partial E^{\geq 2}$ and $\sigma_{E}(e)=r(e)$ for $e \in \partial E^{1}$. In other words, $\sigma_{E}(x)=x_{[2, \infty)}$. We have that

$$
\sigma_{E}\left(\partial E^{\geq 1}\right)=\left\{x \in \partial E \mid E^{1} s(x) \neq \emptyset\right\}=\partial E \backslash\left(\bigcup_{E^{1} v \neq \emptyset} Z(v)\right),
$$

which is an open set, and we see that $\sigma_{E}$ is surjective if and only if $E$ has no sources. We let $\sigma_{E}^{n}: \partial E^{\geq n} \rightarrow \partial E$ be the $n$-fold composition of $\sigma_{E}$ with itself, and we set $\sigma_{E}^{0}=\operatorname{id}_{\partial E}$. Each $\sigma_{E}^{n}$ is then a local homeomorphism between open subsets of $\partial E$. Note that an infinite path $x \in E^{\infty}$ is eventually periodic if and only if there are distinct numbers $m, n \in \mathbb{N}_{0}$ such that $\sigma_{E}^{m}(x)=\sigma_{E}^{n}(x)$.

\subsection{Graph groupoids and their properties}

The graph groupoid of a graph $E$ is the (generalized) Renault-Deaconu groupoid [19] 60] of the dynamical system $\left(\partial E, \sigma_{E}\right)$, that is

$$
\mathcal{G}_{E}:=\left\{(x, m-n, y) \mid m, n \in \mathbb{N}_{0}, x \in \partial E^{\geq m}, y \in \partial E^{\geq n}, \sigma_{E}^{m}(x)=\sigma_{E}^{n}(y)\right\}
$$

as a set. The groupoid structure is given by $(x, k, y) \cdot(y, l, z):=(x, k+l, z)$ (and undefined otherwise), $(x, k, y)^{-1}:=(y,-k, x)$. Thus $\mathcal{G}_{E}^{(0)}=\{(x, 0, x) \mid x \in \partial E\}$, which we will identify with $\partial E$ via $(x, 0, x) \leftrightarrow x$. Then $s(x, k, y)=y$ and $r(x, k, y)=x$. We equip $\mathcal{G}_{E}$ with the topology generated by the basic sets

$$
Z(U, m, n, V):=\left\{(x, m-n, y) \mid x \in U, y \in V, \sigma_{E}^{m}(x)=\sigma_{E}^{n}(y)\right\},
$$

where $U \subseteq \partial E^{\geq m}$ and $V \subseteq \partial E^{\geq n}$ are open sets such that $\left(\sigma_{E}^{m}\right)_{\mid U}$ and $\left(\sigma_{E}^{n}\right)_{\mid V}$ are injective, and $\sigma_{E}^{m}(U)=\sigma_{E}^{n}(V)$. This makes $\mathcal{G}_{E}$ an étale groupoid, and the identification of the unit space with $\partial E$ is compatible with the topology on $\partial E$. Note however, that this topology on $\mathcal{G}_{E}$ is finer than the relative topology induced from $\partial E \times \mathbb{Z} \times \partial E$. According to [10, p. 394] the family

$$
\left\{Z(U,|\mu|,|\lambda|, V) \mid \sigma_{E}^{|\mu|}(U)=\sigma_{E}^{|\lambda|}(V)\right\}
$$

parametrized over all $\mu, \lambda \in E^{*}$ with $r(\mu)=r(\lambda), U \subseteq Z(\mu)$ and $V \subseteq Z(\lambda)$ compact open, is also a basis for the same topology. Each set $Z(U,|\mu|,|\lambda|, V)$ is a compact 
open bisection, and they separate the elements of $\mathcal{G}_{E}$, so $\mathcal{G}_{E}$ is an ample Hausdorff groupoid. The family in (8.1) is countable precisely when $E$ is countable, and so the graph groupoid $\mathcal{G}_{E}$ is second countable exactly when $E$ is countable.

For a boundary path $x \in \partial E$, the isotropy group of $(x, 0, x) \in \mathcal{G}_{E}^{(0)}$ is nontrivial if and only if $x$ is eventually periodic (and infinite). For graph groupoids, effectiveness coincides with topological principality (even without assuming second countability), which in turn is well-known to coincide with the graph satisfying Condition $(L)$.

Proposition 8.2 (cf. [10, Proposition 2.3]). Let E be a graph. The following are equivalent:

(1) The groupoid $\mathcal{G}_{E}$ is effective.

(2) The groupoid $\mathcal{G}_{E}$ is topologically principal.

(3) The set of boundary paths which are not eventually periodic form a dense subset of the boundary path space $\partial E$.

(4) The graph E satisfies Condition $(L)$.

Proof. The equivalence of (2)-(4) is proved (for countable graphs) in [10, Proposition 2.3]. The proof does not rely on the countability of the graph. As it is always the case that (2) implies (1) (cf. Remark 2.2), we only have to verify that (1) implies (4). To that end, assume that $E$ does not satisfy Condition $(L)$. Then there is a cycle $\lambda \in E^{*}$ with no exit, and $\lambda^{\infty}$ is an isolated point in $\partial E$. But then the bisection

$$
Z\left(Z\left(\lambda^{2}\right),|\lambda|^{2},|\lambda|, Z(\lambda)\right)=\left\{\left(\lambda^{\infty},|\lambda|, \lambda^{\infty}\right)\right\}
$$

is an open subset of $\mathcal{G}_{E} \backslash \mathcal{G}_{E}^{(0)}$, and hence $\mathcal{G}_{E}$ is not effective.

We end this subsection by giving a characterization of minimality for graph groupoids. Let $E$ be a graph. Two infinite paths $x, y \in E^{\infty}$ are called tail equivalent if there are natural numbers $k, l$ such that $x_{[k, \infty)}=y_{[l, \infty)}$. Similarly, two finite paths $\mu, \lambda \in E^{*}$ are tail equivalent if $r(\mu)=r(\lambda)$. From the definition of $\mathcal{G}_{E}$ one sees that two boundary paths belong to the same $\mathcal{G}_{E}$-orbit if and only if they are tail equivalent. By combining [8, Theorem 5.1] with [22, Corollary 2.15], we arrive at the following result — of which we provide a self-contained proof.

Proposition 8.3. Let $E$ be a graph. Then the following are equivalent:

(1) The groupoid $\mathcal{G}_{E}$ is minimal.

(2) The graph $E$ is cofinal, and for each $v \in E^{0}$ and $w \in E_{\text {sing }}^{0}$, we have $v \geq w$.

Proof. If $E$ has a sink $w \in E_{\text {sing }}^{0}$, then one immediately deduces from both statements that $E$ cannot have any other singular vertices, nor any infinite paths. 
Consequently

$$
\partial E=\operatorname{Orb}_{\mathcal{G}_{E}}(w)=\left\{\mu \in E^{*} \mid r(\mu)=w\right\},
$$

and this entails that $\mathcal{G}_{E}$ is a discrete transitive groupoid. Now, (1) and (2) are clearly equivalent in this case.

For the remainder of the proof, we assume that $E$ has no sinks. Assume that (2) holds. Let $x \in E^{\infty}$ and let $\lambda \in E^{*}$. By cofinality, there is a path $\lambda^{\prime}$ from $r(\lambda)$ to $s\left(x_{n}\right)$ for some $n \in \mathbb{N}$. The infinite path $\lambda \lambda^{\prime} x_{n} x_{n+1} \ldots$ then belongs to both $Z(\lambda)$ and $\operatorname{Orb}_{\mathcal{G}_{E}}(x)$. Hence the latter is dense in $\partial E$ (since every open set contains a cylinder set when there are no sinks). Next, suppose $\mu \in \partial E \cap E^{*}$ with $r(\mu)$ an infinite emitter. By assumption there is a path $\lambda^{\prime \prime}$ from $r(\lambda)$ to $r(\mu)$, and then $\lambda \lambda^{\prime \prime} \in Z(\lambda) \cap \operatorname{Orb}_{\mathcal{G}_{E}}(\mu)$. This shows that $\mathcal{G}_{E}$ is minimal.

Assume now that $\mathcal{G}_{E}$ is minimal. To see that $E$ is cofinal, let $x \in E^{\infty}$ and $v \in E^{0}$ be given. By minimality there is a $y \in E^{\infty}$ tail equivalent to $x$ such that $y \in Z(v)$. This implies that $v$ can reach $x$. As for the second part of (2), let $v \in E^{0}$ and $w \in E_{\text {sing }}^{0}$ be given. Again by minimality there is a $\lambda \in E^{*} \cap Z(v)$ tail equivalent to $w$, but this is just a path from $v$ to $w$, so $v \geq w$.

Remark 8.4. The notion of cofinality is slightly weaker than strong connectedness. But for finite graphs with no sinks and no sources, cofinality coincides with strong connectedness. In fact, this is also true for infinite graphs which additionally have no semi-heads (the direction-reversed notion of a semi-tail). We also remark that for cofinal graphs, Condition $(L)$ is equivalent to Condition $(K)$.

\section{Topological Full Groups of Graph Groupoids}

We are now going to describe the elements in the topological full group of a graph groupoid. Some examples will be given at the end of the section. We begin by specifying yet another (equivalent) basis for $\mathcal{G}_{E}$, which in turn will allow us to describe bisections combinatorially in terms of the graph.

For two finite paths $\mu, \lambda \in E^{*}$ with $r(\mu)=r(\lambda)=v$ we define

$$
Z(\mu, \lambda):=Z(Z(\mu),|\mu|,|\lambda|, Z(\lambda)) \text {. }
$$

More generally, given a finite subset $F \subseteq v E^{1}$ as well, we define

$$
Z(\mu, F, \lambda):=Z(Z(\mu \backslash F),|\mu|,|\lambda|, Z(\lambda \backslash F)) .
$$

Each $Z(\mu, F, \lambda)$ is a compact open bisection in $\mathcal{G}_{E}$, and we will see shortly that they also form a basis. Observe that if $v \in E_{\mathrm{reg}}^{0}$, then $Z(\mu, F, \lambda)=\bigsqcup_{e \in v E^{1} \backslash F} Z(\mu e, \lambda e)$, and that this is a finite union.

Lemma 9.1. Let $E$ be a graph. Let $\mu, \mu^{\prime}, \lambda, \lambda^{\prime} \in E^{*}$ be finite paths such that $r(\mu)=r(\lambda)=v, r\left(\mu^{\prime}\right)=r\left(\lambda^{\prime}\right)=v^{\prime}$ and let $F \subseteq_{\text {finite }} v E^{1}, F^{\prime} \subseteq_{\text {finite }} v^{\prime} E^{1}$. Then $Z(\mu, F, \lambda) \cap Z\left(\mu^{\prime}, F^{\prime}, \lambda^{\prime}\right)$ equals either

(1) $\emptyset$, or 
(2) $Z(\mu, F, \lambda)$, or

(3) $Z\left(\mu^{\prime}, F^{\prime}, \lambda^{\prime}\right)$, or

(4) $Z\left(\mu, F \cup F^{\prime}, \lambda\right)$, in which case $\mu=\mu^{\prime}, \lambda=\lambda^{\prime}$ and

$$
Z(\mu, F, \lambda) \cup Z\left(\mu^{\prime}, F^{\prime}, \lambda^{\prime}\right)=Z\left(\mu, F \cap F^{\prime}, \lambda\right) .
$$

Proof. Suppose that $Z(\mu, F, \lambda) \cap Z\left(\mu^{\prime}, F^{\prime}, \lambda^{\prime}\right)$ is non-empty. Then, we must have that $|\mu|-|\lambda|=\left|\mu^{\prime}\right|-\left|\lambda^{\prime}\right|, Z(\mu \backslash F) \cap Z\left(\mu^{\prime} \backslash F^{\prime}\right) \neq \emptyset$ and $Z(\lambda \backslash F) \cap Z\left(\lambda^{\prime} \backslash F^{\prime}\right) \neq \emptyset$. Since

$$
Z(\mu \backslash F) \cap Z\left(\mu^{\prime} \backslash F^{\prime}\right)= \begin{cases}Z\left(\mu \backslash\left(F \cup F^{\prime}\right)\right) & \text { if } \mu=\mu^{\prime}, \\ Z(\mu \backslash F) & \text { if } \mu^{\prime}<\mu \text { and } \mu_{\left|\mu^{\prime}\right|+1} \notin F^{\prime}, \\ Z\left(\mu^{\prime} \backslash F^{\prime}\right) & \text { if } \mu<\mu^{\prime} \text { and } \mu_{|\mu|+1}^{\prime} \notin F, \\ \emptyset & \text { otherwise, }\end{cases}
$$

we may suppose without loss of generality, that $\mu \leq \mu^{\prime}$. The equality of $|\mu|-|\lambda|$ and $\left|\mu^{\prime}\right|-\left|\lambda^{\prime}\right|$ then forces $\lambda \leq \lambda^{\prime}$ as well. If $\mu=\mu^{\prime}$, then we must also have $\lambda=\lambda^{\prime}$ and it is easy to see that (4) holds in this case.

Next, suppose $\mu<\mu^{\prime}$, which forces $\lambda<\lambda^{\prime}$. As the intersections above are nonempty we have $Z\left(\mu^{\prime} \backslash F^{\prime}\right) \subseteq Z(\mu \backslash F)$ and $Z\left(\lambda^{\prime} \backslash F^{\prime}\right) \subseteq Z(\lambda \backslash F)$. It follows from this that $Z\left(\mu^{\prime}, F^{\prime}, \lambda^{\prime}\right) \subseteq Z(\mu, F, \lambda)$, and we are done.

Lemma 9.2. The family

$$
\left\{Z(\mu, F, \lambda) \mid \mu, \lambda \in E^{*}, r(\mu)=r(\lambda), F \subseteq \text { finite } r(\mu) E^{1}\right\}
$$

forms a basis for the topology on $\mathcal{G}_{E}$.

Proof. It suffices to write each basic set $Z(U,|\mu|,|\lambda|, V)$, where $\mu, \lambda \in E^{*}$ are finite paths with $r(\mu)=r(\lambda), U \subseteq Z(\mu)$ compact open, $V \subseteq Z(\lambda)$ compact open and $\sigma_{E}^{|\mu|}(U)=\sigma_{E}^{|\lambda|}(V)$, as a union of $Z\left(\mu^{\prime}, F^{\prime}, \lambda^{\prime}\right)$ 's. Given such a basic set, we can write

$$
\sigma_{E}^{|\mu|}(U)=\sigma_{E}^{|\lambda|}(V)=\bigsqcup_{i=1}^{k} Z\left(\eta_{i} \backslash F_{i}\right),
$$

for some $\eta_{i} \in E^{*}, F_{i} \subseteq_{\text {finite }} r\left(\eta_{i}\right) E^{1}$, since the former two are compact open subsets of $\partial E$. It follows that

$$
U=\bigsqcup_{i=1}^{k} Z\left(\mu \eta_{i} \backslash F_{i}\right) \quad \text { and } \quad V=\bigsqcup_{i=1}^{k} Z\left(\lambda \eta_{i} \backslash F_{i}\right)
$$

Hence

$$
Z(U,|\mu|,|\lambda|, V)=\bigsqcup_{\substack{i=1 \\ 1950018-40}}^{k} Z\left(\mu \eta_{i}, F_{i}, \lambda \eta_{i}\right)
$$


Using the basis above, we may concretely describe bisections in $\mathcal{G}_{E}$ as follows.

Lemma 9.3. Let $E$ be graph, and let $U \subseteq \mathcal{G}_{E}$ be a compact open bisection such that $s(U)=r(U)$. Then $U$ is of the form

$$
U=\bigsqcup_{i=1}^{k} Z\left(\mu_{i}, F_{i}, \lambda_{i}\right)
$$

where $\mu_{i}, \lambda_{i} \in E^{*}$ with $r\left(\mu_{i}\right)=r\left(\lambda_{i}\right), F_{i} \subseteq_{\text {finite }} r\left(\mu_{i}\right) E^{1}$ and

$$
s(U)=\bigsqcup_{i=1}^{k} Z\left(\lambda_{i} \backslash F_{i}\right)=\bigsqcup_{i=1}^{k} Z\left(\mu_{i} \backslash F_{i}\right) .
$$

Proof. Since $U$ is a compact open subset of $\mathcal{G}_{E}$ we may, by the preceding two lemmas, write $U$ as a finite disjoint union of basic sets $Z(\mu, F, \lambda)$ 's, say $U=\bigsqcup_{i=1}^{k} Z\left(\mu_{i}, F_{i}, \lambda_{i}\right)$. As $r$ and $s$ are injective on $U$ they preserve disjoint unions, so we have

$$
\begin{aligned}
s(U)=s\left(\bigsqcup_{i=1}^{k} Z\left(\mu_{i}, F_{i}, \lambda_{i}\right)\right)=\bigsqcup_{i=1}^{k} s\left(Z\left(\mu_{i}, F_{i}, \lambda_{i}\right)\right)=\bigsqcup_{i=1}^{k} Z\left(\lambda_{i} \backslash F_{i}\right) \\
=r(U)=r\left(\bigsqcup_{i=1}^{k} Z\left(\mu_{i}, F_{i}, \lambda_{i}\right)\right)=\bigsqcup_{i=1}^{k} r\left(Z\left(\mu_{i}, F_{i}, \lambda_{i}\right)\right)=\bigsqcup_{i=1}^{k} Z\left(\mu_{i} \backslash F_{i}\right) .
\end{aligned}
$$

In conjunction with Lemma 3.7 , we get that the elements in $\llbracket \mathcal{G}_{E} \rrbracket$ for an effective graph groupoid (i.e. the graph $E$ satisfying Condition $(L))$ may be described as follows, in terms of $E$.

Proposition 9.4. Let $E$ be a graph satisfying Condition $(L)$. If $\pi_{U} \in \llbracket \mathcal{G}_{E} \rrbracket$, then the full bisection $U$ can be written as

$$
U=\left(\bigsqcup_{i=1}^{k} Z\left(\mu_{i}, F_{i}, \lambda_{i}\right)\right) \sqcup\left(\partial E \backslash \operatorname{supp}\left(\pi_{U}\right)\right),
$$

where $\mu_{i}, \lambda_{i} \in E^{*}$ with $r\left(\mu_{i}\right)=r\left(\lambda_{i}\right), F_{i} \subsetneq$ finite $r\left(\mu_{i}\right) E^{1}$ and

$$
\operatorname{supp}\left(\pi_{U}\right)=\bigsqcup_{i=1}^{k} Z\left(\lambda_{i} \backslash F_{i}\right)=\bigsqcup_{i=1}^{k} Z\left(\mu_{i} \backslash F_{i}\right) .
$$

Moreover, $\mu_{1}, \ldots \mu_{k}$ are pairwise disjoint, $\lambda_{1}, \ldots, \lambda_{k}$ are pairwise disjoint, and $\mu_{i}, \lambda_{i}$ are distinct for each $1 \leq i \leq k$. The homeomorphism $\pi_{U}: \partial E \rightarrow \partial E$ is given by $x=\lambda_{i} z \mapsto \mu_{i} z$ for $x \in Z\left(\lambda_{i} \backslash F_{i}\right)$ and $x \mapsto x$ otherwise.

Remark 9.5. The elements in $\llbracket \mathcal{G}_{E} \rrbracket$ may alternatively be described in more dynamical terms via the orbits by the shift map. From [10 Proposition 3.3] one deduces that a homeomorphism $\alpha \in \operatorname{Homeo}(\partial E)$ belongs to $\llbracket \mathcal{G}_{E} \rrbracket$ if and only if 
there are compactly supported continuous functions $m, n: \partial E \rightarrow \mathbb{N}_{0}$ such that $\sigma_{E}^{m(x)}(\alpha(x))=\sigma_{E}^{n(x)}(x)$. This parallels Matui's definition for locally compact Cantor minimal systems mentioned in Remark [3.3] and Matsumoto's definition for one-sided shifts of finite type in [42].

Having completely described the topological full group of a graph groupoid, we provide an example to show that the assumption on the orbits in Lemma 4.9 is not a necessary condition. On the other hand, we also give an example to show that the statement is generally false without said assumption. These examples also provide examples of densely minimal groupoids which are not minimal.

Example 9.6. Consider the following graph:

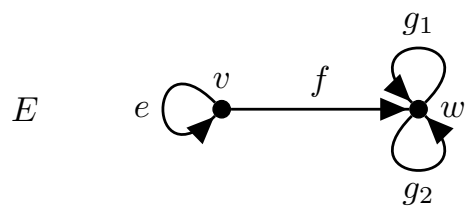

The graph $E$ satisfies condition $(L)$, but is not cofinal, so $\mathcal{G}_{E}$ is effective, but not minimal. We claim that $\mathcal{G}_{E}$ is densely minimal. To see this, note that any nonempty open subset of $E^{\infty}$ must contain a cylinder set $Z(\mu)$ where $r(\mu)=w$. And the restriction of $\mathcal{G}_{E}$ to $Z(\mu)$ is minimal. As for covering, observe that the orbit of $e^{\infty} \in \partial E$ has length one, i.e. $\operatorname{Orb}_{\mathcal{G}_{E}}\left(e^{\infty}\right)=\left\{e^{\infty}\right\}$. However, the topological full group $\llbracket \mathcal{G}_{E} \rrbracket$ still covers $\mathcal{G}_{E}$. For instance, the isotropy element $\left(e^{\infty}, 1, e^{\infty}\right)$ belongs to the full bisection

$$
U=Z\left(e^{2}, e\right) \sqcup Z\left(e f, g_{1} g_{2}\right) \sqcup Z\left(g_{1}, g_{1} g_{1}\right) \sqcup Z(f, f) \sqcup Z\left(g_{2}, g_{2}\right) .
$$

Similar full bisections can be found for $\left(e^{\infty}, k, e^{\infty}\right)$ where $k$ is any integer.

Example 9.7. Consider the following graph:

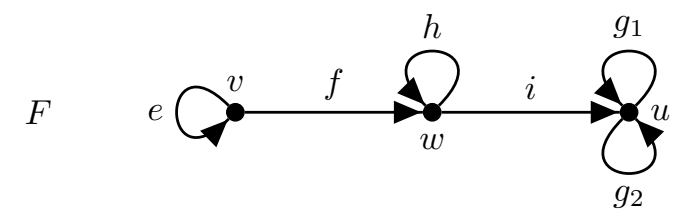

As in the previous example, $e^{\infty} \in \partial F$ has a singleton orbit. However, in contrast to the previous example, $\llbracket \mathcal{G}_{F} \rrbracket$ does not cover $\mathcal{G}_{F}$. For there is no full bisection containing the element $\left(e^{\infty}, 1, e^{\infty}\right)$. If $U$ is a bisection containing $\left(e^{\infty}, 1, e^{\infty}\right)$, then $U$ must contain a bisection of the form $Z\left(e^{k+1}, e^{k}\right)$. But since $Z\left(e^{k}\right)=Z\left(e^{k+1}\right) \sqcup Z\left(e^{k} f\right)$, it will be impossible to enlarge $U$ to a full bisection. By adding disjoint $Z(\mu, \lambda)$ 's in order to write $U$ as in Proposition 9.4 one will always have one more $\mu$ ending in $w$ than $\lambda$ 's. See also [7] Example 3.5] for the same phenomenon in a restricted transformation groupoid. 


\section{Isomorphism Theorems for Graph Groupoids}

In this section, we will pursue specialized isomorphism theorems for graph groupoids. We will determine exactly when the topological full group of a graph groupoid belongs to $K^{F}$, and the conditions for this turn out to be weaker than minimality. We will also determine, in terms of the graph, exactly when it belongs to $K^{\mathrm{LCC}}$. From this we obtain two isomorphism theorems for graph groupoids.

\subsection{The class $K^{F}$}

We are now going to give necessary and sufficient conditions for when $(\Gamma, \partial E)$ belongs to $K^{F}$ — for a graph $E$, and a subgroup $\Gamma \leq \llbracket \mathcal{G}_{E} \rrbracket$ containing $\mathrm{D}\left(\llbracket \mathcal{G}_{E} \rrbracket\right)$. Of the three conditions (F1), (F2) and (F3) in Definition 6.3, (F1) is the "hardest" one to satisfy. This is essentially because we need to produce elements in the topological full group with support containing a given point $x \in \partial E$, but also contained in a given neighborhood of $x$. In the other two conditions, we can get away with simply choosing a "small enough" support. As both conditions (F1) and (F3) fails in the presence of isolated points, we will only consider graphs that have no sinks, no semitails, and satisfy Condition $(L)$. We will see that Condition $(K)$ will be necessary for (F1) to hold for periodic ${ }^{\mathrm{g}}$ points. The two conditions in Definition 10.1 below are needed to ensure that (F1) holds for wandering infinite paths, and for finite boundary paths, respectively. For notational convenience we make the following ad-hoc definitions.

Definition 10.1. Let $E$ be a graph.

(1) We say that $E$ satisfies Condition $(W)$ if for every wandering infinite path $x \in E^{\infty}$, we have $\left|s(x) E^{*} r\left(x_{n}\right)\right| \geq 2$ for some $n \in \mathbb{N}$.

(2) We say that $E$ satisfies Condition $(\infty)$ if for every infinite emitter $v \in E^{0}$, the set $\left\{e \in v E^{1} \mid r(e) \geq v\right\}$ is infinite.

The three conditions $(K),(W)$ and $(\infty)$ can be thought of as strengthenings of each of the three criteria for the boundary path space $\partial E$ being perfect (Proposition 8.1). The latter three criteria can informally be described as "can exit", whereas the former three can be described as "can exit and return". More specifically, Condition $(L)$ means that one can exit every cycle, whereas Condition $(K)$ means that one can also return back to the same cycle. That $E$ has no semi-tails means that every wandering infinite path has an exit, and Condition $(W)$ means that one can return to the same infinite path again. That $E$ has no sinks can be reformulated as saying that every singular vertex has an exit (and hence infinitely many), whereas Condition $(\infty)$ says that one can also return to the same vertex (from infinitely many of these exits). Note that Condition $(\infty)$ holds in particular if every infinite emitter supports infinitely many loops. Also note that if $\left|s(x) E^{*} r\left(x_{n}\right)\right| \geq 2$ for

${ }^{\mathrm{g}}$ That is, $x=\lambda^{\infty}$ for some cycle $\lambda \in E^{*}$. 
some $n \in \mathbb{N}$, then the same is true for each $m \geq n$. We now make two elementary observations needed in the proof of the next proposition.

Lemma 10.2. Let $E$ be a graph.

(1) If $\mu \in E^{*}$ is a cycle and $E$ satisfies Condition $(K)$, then there are infinitely many cycles $\lambda_{1}, \lambda_{2}, \ldots$ based at $s(\mu)$ such that $\mu, \lambda_{1}, \lambda_{2}, \ldots$ are mutually disjoint.

(2) If $x=x_{1} x_{2} \ldots \in E^{\infty}$ is a wandering infinite path and $E$ satisfies Condition $(W)$, then for each $N \in \mathbb{N}$ there is an $n \in \mathbb{N}$ and paths $\mu_{1}, \ldots, \mu_{N}$ from $s(x)$ to $r\left(x_{n}\right)$ such that $x_{[1, n]}, \mu_{1}, \ldots, \mu_{N}$ are mutually disjoint.

Proof. For the first part, let $\tau_{1}$ and $\tau_{2}$ be two distinct return paths based at $s(\mu)$. As distinct return paths are disjoint we must have that $\mu$ is disjoint from one of them, say $\tau_{1}$. And then the cycles $\mu, \tau_{1} \mu, \tau_{1}^{2} \mu, \tau_{1}^{3} \mu, \ldots$ are all disjoint.

We argue inductively for the second part. Let $n_{1}$ be a natural number with $\left|s(x) E^{*} r\left(x_{n_{1}}\right)\right| \geq 2$, and put $v=r\left(x_{n_{1}}\right)$. Since $x$ is wandering we can let $m_{1} \geq n_{1}$ be the largest index such that $r\left(x_{m_{1}}\right)=v$. So that $x$ never returns to $v$ after the $m_{1}$ 'th edge. Let $\mu$ be a path in $s(x) E^{*} r\left(x_{m_{1}}\right)$ distinct from $x_{\left[1, m_{1}\right]}$. If $x_{\left[1, m_{1}\right]}$ and $\mu$ are disjoint, then we are done with the base case. If not, then either $x_{\left[1, m_{1}\right]}<\mu$ or $x_{\left[1, m_{1}\right]}>\mu$. In the former case we have that $\mu=x_{\left[1, m_{1}\right]} \rho$, where $\rho$ is a cycle based at $v$. As $x$ does not return to $v$ again we must have that $x_{\left[m_{1}+1, m_{1}+|\rho|\right]} \neq \rho$, and then $x_{\left[1, m_{1}+|\rho|\right]}$ is disjoint from the path

$$
\mu_{1}:=\mu x_{\left[m_{1}+1, m_{1}+|\rho|\right]}=x_{\left[1, m_{1}\right]} \rho x_{\left[m_{1}+1, m_{1}+|\rho|\right]} .
$$

If the latter is the case, then $\mu=x_{[1, k]}$ for some $k<m_{1}$ and $x_{\left[k+1, m_{1}\right]}$ is a cycle. And then the previous argument applied to $x_{\left[1, m_{1}\right]}$ and $\mu^{\prime}=x_{[1, k]} x_{\left[k+1, m_{1}\right]} x_{\left[k+1, m_{1}\right]}$ shows that the statement holds for $N=1$.

Applying the above to the tail $x_{\left[m_{1}+1, \infty\right)}$, which is again a wandering infinite path, we get an index $m_{2}>m_{1}$ and a path $\mu_{2}$ from $r\left(x_{m_{1}}\right)$ to $r\left(x_{m_{2}}\right)$ disjoint from $x_{\left[m_{1}+1, m_{2}\right]}$. By concatenating $x_{\left[1, m_{1}\right]}$ and $\mu_{1}$ with $x_{\left[m_{1}+1, m_{2}\right]}$ and $\mu_{2}$, we obtain three paths from $s(x)$ to $r\left(x_{m_{2}}\right)$ that are mutually disjoint, as well as disjoint from $x_{\left[1, m_{2}\right]}$. By continuing in this manner one sees that the result is true for all $N \in \mathbb{N}$.

Proposition 10.3. Let $E$ be a graph with no sinks and let $\Gamma \leq \llbracket \mathcal{G}_{E} \rrbracket$ be a subgroup containing $\mathrm{D}\left(\llbracket \mathcal{G}_{E} \rrbracket\right)$. Then $(\Gamma, \partial E)$ belongs to $K^{F}$ if and only if $E$ satisfies Condition $(K),(W)$ and $(\infty)$.

Proof. This proof is inspired by Matui's proof of [49, Proposition 3.6]. We employ similar tricks in this more concrete, yet non-minimal context. We will first show that (F2) and (F3) holds when $E$ satisfies Condition $(K)$ and $(W)$. And then we will show, in turn, that all three conditions are necessary and sufficient for (F1) to hold at certain boundary paths. 
Suppose $E$ satisfies Condition $(K)$ and $(W)$ (in addition to having no sinks). We verify (F3) first. Let $A$ be any non-empty clopen subset of $\partial E$. There is then a path $\eta$ such that $Z(\eta) \subseteq A$. Now there are two possibilities. Either $r(\eta)$ connects to a cycle, or $r(\eta) E^{\infty}$ consists only of wandering paths. In the first case, we may assume, by extending $\eta$, that $r(\eta)$ supports a cycle. By Lemma 10.2, we can find three disjoint cycles $\lambda_{1}, \lambda_{2}, \lambda_{3}$ based at $r(\eta)$. Define $V=Z\left(\eta \lambda_{1}, \eta \lambda_{2}\right)$, $W=Z\left(\eta \lambda_{2}, \eta \lambda_{3}\right)$ and $\alpha=\left[\pi_{\hat{V}}, \pi_{\hat{W}}\right]$ (as in Lemma 3.8). Then $\alpha \in \Gamma \backslash\{1\}$ has order three and $\operatorname{supp}(\alpha) \subseteq Z(\eta) \subseteq A$. In the case that $r(\eta) E^{\infty}$ consists only of wandering paths we may find, again by Lemma 10.2, three disjoint paths $\lambda_{1}, \lambda_{2}, \lambda_{3}$ starting at $r(\eta)$, and such that $r\left(\lambda_{1}\right)=r\left(\lambda_{2}\right)=r\left(\lambda_{3}\right)$. Defining $\alpha$ as above shows that (F3) holds in this case as well.

Next, we verify (F2). To that end, let $\alpha$ be a nontrivial involution in $\Gamma$ and let $A$ be a non-empty clopen subset of $\operatorname{supp}(\alpha)$. We have $\alpha=\pi_{U}$ with

$$
U=\left(\bigsqcup_{i=1}^{k} Z\left(\mu_{i}, F_{i}, \lambda_{i}\right)\right) \sqcup\left(\mathcal{G}_{E}^{(0)} \backslash \operatorname{supp}\left(\pi_{U}\right)\right)
$$

as in Proposition 9.4 Arguing as above, we can find a finite path $\eta$ and an index $1 \leq j \leq k$ such that $Z(\eta) \subseteq A \cap Z\left(\lambda_{j} \backslash F_{j}\right)$, as well as two disjoint paths $\tau_{1}, \tau_{2}$ satisfying $s\left(\tau_{1}\right)=s\left(\tau_{2}\right)=r(\eta)$ and $r\left(\tau_{1}\right)=r\left(\tau_{2}\right)$. As $\lambda_{j} \leq \eta$ we can write $\eta=\lambda_{j} \rho$ for some path $\rho$ whose first edge does not belong to $F_{j}$. Define the bisections

$$
V=Z\left(\lambda_{j} \rho \tau_{1}, \lambda_{j} \rho \tau_{2}\right) \sqcup Z\left(\mu_{j} \rho \tau_{1}, \mu_{j} \rho \tau_{2}\right)
$$

and

$$
W=Z\left(\mu_{j} \rho \tau_{1}, \lambda_{j} \rho \tau_{1}\right) .
$$

Put $\beta=\left[\pi_{\hat{V}}, \pi_{\hat{W}}\right]$. As $\alpha$ is an involution we must have that $\alpha\left(\lambda_{j} z\right)=\mu_{j} z$ for $\lambda_{j} z \in Z\left(\lambda_{j} \backslash F_{j}\right)$ and vice versa. Now observe that $\beta \in \Gamma$,

$$
\begin{aligned}
\operatorname{supp}(\beta) & =Z\left(\lambda_{j} \rho \tau_{1}\right) \sqcup Z\left(\lambda_{j} \rho \tau_{2}\right) \sqcup Z\left(\mu_{j} \rho \tau_{1}\right) \sqcup Z\left(\mu_{j} \rho \tau_{2}\right) \\
& \subseteq Z(\eta) \cup \alpha(Z(\eta)) \subseteq A \cup \alpha(A),
\end{aligned}
$$

and that $\alpha$ and $\beta$ agree on $\operatorname{supp}(\beta)$ (as they both swap the initial paths $\lambda_{j}$ and $\mu_{j}$ ).

Assume now that $E$ merely has no sinks, no semi-tails and satisfies Condition $(L)$. We will show that (F1) holds if and only if $E$ satisfies Condition $(K)$, $(W)$ and $(\infty)$. Let $x \in \partial E$ and $A$ a clopen neighborhood of $x$ be given. We further divide this part into three cases, each one yielding the necessity of one of the three conditions.

Condition $(\boldsymbol{K})$ : Assume $E$ satisfies Condition $(K)$, and suppose $x=x_{1} x_{2} \ldots$ is an infinite non-wandering path. By choosing $m \in \mathbb{N}$ large enough, we can ensure that $Z\left(x_{[1, m]}\right) \subseteq A$. And since $x$ contains infinitely many cycles we can, by possibly choosing $m$ larger, assume that $x_{[m+1, n]}$ is a return path at $r\left(x_{m}\right)$ for some $n>m$. Using Lemma 10.2, we can find three mutually disjoint cycles $\lambda_{1}, \lambda_{2}, \lambda_{3}$ all based 
at $r\left(x_{m}\right)$ which are also disjoint from $x_{[m+1, n]}$. Let $\mu_{i}=x_{[1, m]} \lambda_{i}$ for $i=1,2,3$ and let $\mu_{4}=x_{[1, n]}$. Define

$$
V=Z\left(\mu_{1}, \mu_{2}\right) \sqcup Z\left(\mu_{3}, \mu_{4}\right)
$$

and

$$
W=Z\left(\mu_{1}, \mu_{3}\right)
$$

Then $\alpha=\left[\pi_{\hat{V}}, \pi_{\hat{W}}\right] \in \Gamma$ satisfies $\operatorname{supp}(\alpha)=\bigsqcup_{i=1}^{4} Z\left(\mu_{i}\right) \subseteq Z\left(x_{[1, m]}\right) \subseteq A, \alpha^{2}=1$ and $x \in Z\left(\mu_{4}\right) \subseteq \operatorname{supp}(\alpha)$ as desired.

To see that Condition $(K)$ is necessary, suppose that $E$ does not satisfy it. Then there is a vertex $v \in E^{0}$ supporting a unique return path, say $\tau$. We may assume that $\tau$ has an exit $f$ with $s(f)=v$. Consider $x=\tau^{\infty}$ and its neighborhood $A=Z(\tau)$. We claim that (F1) fails for this pair. To see this, suppose $\pi_{U} \in \llbracket \mathcal{G}_{E} \rrbracket$ satisfies $\tau^{\infty} \in \operatorname{supp}\left(\pi_{U}\right) \subseteq Z(\tau)$. By Proposition 9.4 we can find $Z(\mu, \lambda) \subseteq U$ with $r(\mu)=r(\lambda), \mu \neq \lambda$ and $\tau^{\infty} \in Z(\lambda)$, which means that $\lambda \leq \tau^{k}$ for some $k \geq 1$. By possibly extending $\mu$ and $\lambda$ we may assume that $\lambda=\tau^{k}$. We also have $Z(\mu) \subseteq Z(\tau)$, i.e. $\tau \leq \mu$, and $r(\mu)=r(\lambda)=v$. But since $\tau$ is the only return path based at $v$ we must have $\mu=\tau^{l}$ for some $l \neq k$ as $\mu \neq \lambda$. Let $z \in r(f) \partial E$. Then $\left(\pi_{U}\right)^{2}\left(\tau^{2 k} f z\right)=\tau^{2 l} f z \neq \tau^{2 k} f z$, hence $\pi_{U}$ is not an involution, and therefore $(\Gamma, \partial E)$ does not satisfy $(\mathrm{F} 1)$.

Condition $(\boldsymbol{W})$ : Assume $E$ satisfies Condition $(W)$, and suppose $x=x_{1} x_{2} \ldots$ is an infinite wandering path. Choose $m$ large enough so that $Z\left(x_{[1, m]}\right) \subseteq A$. By Lemma 10.2 there is an $n \geq m$ and three paths $\lambda_{1}, \lambda_{2}, \lambda_{3}$ from $s(x)$ to $r\left(x_{n}\right)$ such that $\lambda_{1}, \lambda_{2}, \lambda_{3}, x_{[1, n]}$ are mutually disjoint. Setting $\mu_{i}=x_{[1, m]} \lambda_{i}$ for $i=1,2,3$ and $\mu_{4}=x_{[1, n]}$, and defining $\alpha$ in the same way as in the case of Condition $(K)$ above gives the desired element in $\Gamma$.

To see that Condition $(W)$ is necessary, suppose there is an infinite wandering path $x=x_{1} x_{2} \ldots$ such that $\left|s(x) E^{*} s\left(x_{n}\right)\right|=1$ for all $n \in \mathbb{N}$. We claim that (F1) fails for $A=Z\left(x_{1}\right)$. Indeed, suppose $\pi_{U} \in \llbracket \mathcal{G}_{E} \rrbracket$ satisfies $x \in \operatorname{supp}\left(\pi_{U}\right) \subseteq Z\left(x_{1}\right)$. By Proposition 9.4, we can find $Z(\mu, \lambda) \subseteq U$ with $r(\mu)=r(\lambda), \mu \neq \lambda$ and $x \in Z(\lambda)$, which implies that $\lambda=x_{[1, m]}$ for some $m \geq 1$. But as $Z(\mu) \subseteq Z\left(x_{1}\right)$ we have that $s(\mu)=s(x)$ and $r(\mu)=r\left(x_{m}\right)$. It now follows that $\mu=\lambda$ since $\left|s(x) E^{*} s\left(x_{m}\right)\right|=1$. This contradiction shows that there is not even an element $\pi_{U} \in \llbracket \mathcal{G}_{E} \rrbracket$ such that $x \in \operatorname{supp}\left(\pi_{U}\right) \subseteq Z\left(x_{1}\right)$.

Condition ( $\infty)$ : Assume $E$ satisfies Condition $(\infty)$, and suppose $x=x_{1} \ldots x_{m}$ is a finite boundary path. Then for some $F \subseteq$ finite $r(x) E^{1}$ we have $Z(x \backslash F) \subseteq A$. By Condition $(\infty)$ we can find three distinct edges $e_{1}, e_{2}, e_{3} \in r(x) E^{1} \backslash F$, and three (necessarily disjoint) cycles $\tau_{1}, \tau_{2}, \tau_{3}$ based at $r(x)$ such that $e_{i} \leq \tau_{i}$ for $i=1,2,3$. Let $F^{\prime}=F \sqcup\left\{e_{1}, e_{2}, e_{3}\right\}$. Now define

$$
V=Z\left(x \tau_{1}, F^{\prime}, x\right) \sqcup Z\left(x \tau_{2}, F^{\prime}, x \tau_{3}\right)
$$


and

$$
W=Z\left(x \tau_{1}, F^{\prime}, x \tau_{2}\right) .
$$

Then $\alpha=\left[\pi_{\hat{V}}, \pi_{\hat{W}}\right]$ satisfies $\operatorname{supp}(\alpha)=Z\left(x \backslash F^{\prime}\right) \bigsqcup_{i=1}^{3} Z\left(x \tau_{i} \backslash F^{\prime}\right) \subseteq Z(x \backslash F) \subseteq A$, $\alpha^{2}=1$ and $x \in Z\left(x \backslash F^{\prime}\right) \subseteq \operatorname{supp}(\alpha)$.

Finally, if $E$ does not satisfy Condition $(\infty)$, then there is an infinite emitter $v \in E^{0}$ such that the set $F=\left\{e \in v E^{1} \mid r(e) \geq v\right\}$ is finite. And then (F1) fails for $x=v$ and $A=Z(v \backslash F)$ as there is no element $\pi_{U} \in \llbracket \mathcal{G}_{E} \rrbracket$ whose support is contained in $Z(v \backslash F)$ and contains $v$. The argument for this is essentially the same as in the necessity of Condition $(W)$ above.

Remark 10.4. From Proposition 10.3 we see that for a graph groupoid $\mathcal{G}_{E}$, the topological full group $\llbracket \mathcal{G}_{E} \rrbracket$ (on the boundary path space $\partial E$ ) belongs to the class $K^{F}$ if and only if its commutator subgroup $\mathrm{D}\left(\llbracket \mathcal{G}_{E} \rrbracket\right)$ does. This is not something one would expect in general from the definition of $K^{F}$. It is clear that $(\mathrm{F} 1)$ and (F3) in Definition 6.3 passes to supergroups, but (F2) need not do so. It is even more peculiar that the properties (F1)-(F3) pass down to the commutator from $\llbracket \mathcal{G}_{E} \rrbracket$. This phenomenon might be an artifact of the combinatorial nature of the topological full group of a graph groupoid, and so it might also hold for other concrete classes of groupoids.

\subsection{The class $K^{\mathrm{LCC}}$}

Our next objective is to perform a similar analysis of when the space-group pair $\left(\llbracket \mathcal{G}_{E} \rrbracket, \partial E\right)$ for a graph $E$ belongs to $K^{\mathrm{LCC}}$. In this case the "mixing conditions" will be weaker than for $K^{F}$ (cf. Proposition 10.3), but we are only able to prove membership for the topological full group itself - no proper subgroups. As in the case of $K^{F}$ we need to stipulate that the boundary path space $\partial E$ has no isolated points (cf. condition (K1) in Definition 6.18), but also that the graphs are countable (this also for condition (K1)). By the results in Sec. 7, we only have to determine when $\mathcal{G}_{E}$ is non-wandering, and when all orbits have length at least three. We shall soon see that the former property is characterized by excluding certain "tree-like" components in the graph $E$, which we make precise in the following definition.

Definition 10.5. We say that a graph $E$ satisfies Condition $(T)$ if for every vertex $v \in E^{0}$, there exists a vertex $w \in E^{0}$ such that $\left|v E^{*} w\right| \geq 2$.

Note that Condition $(T)$ implies that there are no sinks and no semi-tails. It does not, however, imply Condition $(L)$ as one can traverse a cycle twice to get two different paths. As long as there are no sinks, Condition $(W)$ implies Condition $(T)$. Condition $(T)$ is a fairly weak condition; it is in fact satisfied by all graphs that have finitely many vertices and no sinks, and more generally by any graph in which every vertex connects to a cycle. The archetypical example of graphs not satisfying Condition $(T)$ are trees, or more generally graphs containing such components. 
As for when $\mathcal{G}_{E}$ can have orbits of length one or two one finds, by merely exhausting all possibilities, that this happens exactly if one or more of the following kinds of vertices are present in the graph $E$.

Definition 10.6. Let $E$ be a graph. We say that a vertex $v \in E^{0}$ is degenerate if it is one of the following types:

(1) "1-loop-source": $E^{1} v=\{e\}$ where $e$ is a loop.

(2) "1 source to 1 -loop-source": $E^{1} v=\{e, f\}$ where $e$ is a loop and $s(f)$ is a source.

(3) "2-loop-source": There is another vertex $w \in E^{0}$ distinct from $v$ such that $E^{1} v=\{e\}=w E^{1} v$ and $E^{1} w=\{f\}=v E^{1} w$.

(4) "Infinite source": $v E^{1}$ is infinite and $E^{1} v$ is empty.

(5) " 1 source to singular": $v$ is singular and $E^{1} v=\{f\}$ where $s(f)$ is a source.

(6) "Stranded": $v E^{1}$ and $E^{1} v$ are both empty.

Proposition 10.7. Let $E$ be a graph.

(1) $\mathcal{G}_{E}$ is non-wandering if and only if E satisfies Condition $(L)$ and $(T)$.

(2) $\left|\operatorname{Orb}_{\mathcal{G}_{E}}(x)\right| \geq 3$ for all $x \in \partial E$ if and only if $E$ has no degenerate vertices.

Proof. We prove part (1) first. We may assume that $E$ has no sinks, as this is implied by both of the statements in (1). Suppose $E$ satisfies Condition $(L)$ and $(T)$. Let $A$ be a non-empty clopen subset of $\partial E$. Then there is a path $\mu \in E^{*}$ such that $Z(\mu) \subseteq A$. Suppose first that $r(\mu)$ connects to a cycle. Let $\lambda$ be such a cycle and let $\rho$ be a path from $r(\mu)$ to $s(\lambda)$. We may assume that $\lambda$ has an exit $f$ with $s(f)=s(\lambda)$. Let $x \in r(f) E^{\infty}$. Then $\mu \rho f x$ and $\mu \rho \lambda f x$ are two distinct tailequivalent boundary paths in $A$. If, on the other hand, $r(\mu)$ does not connect to a cycle, then $r(\mu) E^{\infty}$ consists only of wandering paths that visit each vertex at most once. Let $w \in E^{0}$ be a vertex such that there are two distinct paths $\rho_{1}, \rho_{2}$ from $r(\mu)$ to $w$. Again letting $x \in w E^{\infty}$ be arbitrary we have that $\mu \rho_{1} x$ and $\mu \rho_{2} x$ are two distinct tail-equivalent boundary paths in $A$. Hence $A$ is not wandering.

To see that Condition $(L)$ and $(T)$ are both necessary, note first that if $E$ does not satisfy Condition $(L)$, then $\partial E$ has an isolated point, and a clopen singleton is surely wandering. Assume instead that $E$ fails to satisfy Condition $(T)$, and let $v \in E^{0}$ be a vertex such that there is either no path or a unique path from $v$ to any other vertex in $E$. We claim that the cylinder set $Z(v)$ is wandering. We first consider a finite boundary path $\mu$ beginning in $v$ (if such a path exists). Then $r(\mu)$ is a singular vertex and

$$
\operatorname{Orb}_{\mathcal{G}_{E}}(\mu) \cap Z(v)=\left\{\lambda \in E^{*} \mid s(\lambda)=v, r(\lambda)=r(\mu)\right\}=v E^{*} r(\mu)=\{\mu\},
$$

as desired. Similarly, if $x \in v E^{\infty}$ and $y \in \operatorname{Orb}_{\mathcal{G}_{E}}(x) \cap Z(v)$, then there are $k, l \in \mathbb{N}$ such that $x_{[k, \infty)}=y_{[l, \infty)}$. In particular $x_{[1, k-1]}$ and $y_{[1, l-1]}$ are finite paths from $v$ to $s\left(x_{k}\right)=s\left(y_{l}\right)$, hence these are equal and it follows then that $x=y$. Therefore $\operatorname{Orb}_{\mathcal{G}_{E}}(x) \cap Z(v)=\{x\}$. This proves the first part of the proposition. 
For part (2), simply note that an orbit of length one can only occur if there are degenerate vertices of type (1), (4) or (6) as in Definition 10.6 (the corresponding orbits of length one being $\left\{e^{\infty}\right\},\{v\},\{v\}$, respectively). And that an orbit of length two can only occur if there are degenerate vertices of type (2), (3) or (5) (the corresponding orbits of length two being $\left\{e^{\infty}, f e^{\infty}\right\},\left\{(e f)^{\infty},(f e)^{\infty}\right\},\{v, f\}$, respectively).

Remark 10.8. By an argument as in Example 9.6 one deduces that if a graph $E$ satisfies Condition $(I)$, then the graph groupoid $\mathcal{G}_{E}$ is densely minimal. However, statement (1) in Proposition 10.7 is strictly weaker than $\mathcal{G}_{E}$ being densely minimal. It is easy to cook up examples of infinite graphs satisfying Condition $(L)$ and $(T)$, but whose graph groupoids are not densely minimal. One such example is

E

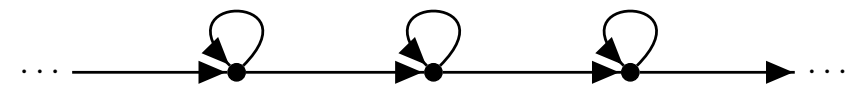

\subsection{Isomorphism theorems}

Recall that all orbits having length at least three is sufficient for the commutator subgroup of the topological full group to cover the groupoid (Lemma 4.9). This in turn means that the groupoid can be recovered as the groupoid of germs of any subgroup between the topological full group and its commutator. Combined with Propositions 10.3 and 10.7, we will obtain the two isomorphism results for graph groupoids. We begin by first observing that the conditions on the graph for membership in $K^{F}$ actually implies that all orbits are infinite.

Lemma 10.9. Let $E$ be a graph with no sinks and suppose $E$ satisfies Condition $(K)$ and $(\infty)$. Then $\operatorname{Orb}_{\mathcal{G}_{E}}(x)$ is infinite for each $x \in \partial E$. In particular, $E$ has no degenerate vertices.

Proof. We first consider the $\mathcal{G}_{E}$-orbits of finite boundary paths. Suppose $v \in E^{0}$ is an infinite emitter. Condition $(\infty)$ implies that there are infinitely many distinct return paths at $v$, hence $\operatorname{Orb}_{\mathcal{G}_{E}}(\mu)$ is infinite for each $\mu \in \partial E \cap E^{*}$.

Next, let $x \in E^{\infty}$ be an infinite path. If $x$ is eventually periodic, then $x=\mu \lambda^{\infty}$ for some finite path $\mu$ and some cycle $\lambda$. Lemma 10.2 gives a sequence of mutually disjoint cycles $\tau_{1}, \tau_{2}, \ldots$ based at $s(\lambda)$. And then $\left\{\tau_{1} \lambda^{\infty}, \tau_{2} \lambda^{\infty}, \ldots\right\}$ is an infinite subset of $\operatorname{Orb}_{\mathcal{G}_{E}}(x)$. If $x$ is not eventually periodic, then $\left\{x, x_{[2, \infty]}, x_{[3, \infty]}, \ldots\right\}$ is an infinite subset of $\operatorname{Orb}_{\mathcal{G}_{E}}(x)$.

In terms of the class $K^{F}$, we obtain the following isomorphism result, which relaxes the assumptions in Theorem 7.2 considerably for graph groupoids.

Theorem 10.10. Let $E$ and $F$ be graphs with no sinks, and suppose they both satisfy Condition $(K),(W)$ and $(\infty)$. Suppose $\Gamma \leq \llbracket \mathcal{G}_{E} \rrbracket$ and $\Lambda \leq \llbracket \mathcal{G}_{F} \rrbracket$ are subgroups containing $\mathrm{D}\left(\llbracket \mathcal{G}_{E} \rrbracket\right)$ and $\mathrm{D}\left(\llbracket \mathcal{G}_{F} \rrbracket\right)$, respectively. If $\Gamma \cong \Lambda$ as abstract groups, 
then $\mathcal{G}_{E} \cong \mathcal{G}_{F}$ as topological groupoids. In particular, the following are equivalent:

(1) $\mathcal{G}_{E} \cong \mathcal{G}_{F}$ as topological groupoids.

(2) $\llbracket \mathcal{G}_{E} \rrbracket \cong \llbracket \mathcal{G}_{F} \rrbracket$ as abstract groups.

(3) $\mathrm{D}\left(\llbracket \mathcal{G}_{E} \rrbracket\right) \cong \mathrm{D}\left(\llbracket \mathcal{G}_{F} \rrbracket\right)$ as abstract groups.

Proof. Combine Proposition [10.3. Theorem [6.6] Proposition 6.2] Lemma [10.9, Lemma 4.9 and Proposition 4.10.

The preceding result covers - in particular - all finite graphs that have no sinks and satisfy Condition $(K)$. As for an isomorphism result in terms of $K^{\mathrm{LCC}}$, we combine Proposition 10.7 with Theorem 7.10 to get the following result.

Theorem 10.11. Let $E$ and $F$ be countable graphs satisfying Condition $(L)$ and $(T)$, and having no degenerate vertices. Then the following are equivalent:

(1) $\mathcal{G}_{E} \cong \mathcal{G}_{F}$ as topological groupoids.

(2) $\llbracket \mathcal{G}_{E} \rrbracket \cong \llbracket \mathcal{G}_{F} \rrbracket$ as abstract groups.

This result covers - in particular - all finite graphs that have no degenerate vertices nor sinks and which satisfy Condition $(L)$.

Remark 10.12. In [43], Matsumoto established a version of Theorem 10.11 for finite graphs which are strongly connected (and satisfy Condition $(L)$, or equivalently $(K))$. At about the same time, Matui announced [49, and his Isomorphism Theorem therein applies to the enlarged class of graphs which have finitely many vertices, countably many edges, no sinks, are cofinal, satisfy Condition $(L)$ and for which every vertex can reach every infinite emitter.

Combining Theorem 10.11 with [10, Theorem 5.1] and [13, Corollary 4.2], we obtain the rigidity result in Corollary 10.13 below, which ties in many of the mathematical structures associated to (directed) graphs. For background on graph $C^{*}$-algebras, see [58, ${ }^{\mathrm{h}}$ and for Leavitt path algebras, see 1].

Corollary 10.13. Let $E$ and $F$ be countable graphs satisfying Condition $(L)$ and $(T)$, and having no degenerate vertices. Let $R$ be an integral domain. Then the following are equivalent:

(1) The graph groupoids $\mathcal{G}_{E}$ and $\mathcal{G}_{F}$ are isomorphic as topological groupoids.

(2) There is an isomorphism of the graph $C^{*}$-algebras $C^{*}(E)$ and $C^{*}(F)$ which maps the diagonal $\mathcal{D}(E)$ onto $\mathcal{D}(F)$.

(3) There is an isomorphism of the Leavitt path algebras $L_{R}(E)$ and $L_{R}(F)$ which maps the diagonal $D_{R}(E)$ onto $D_{R}(F)$.

\footnotetext{
${ }^{\mathrm{h}}$ Beware that the convention for paths in graphs in Raeburn's book is opposite of the one used in this paper.
} 
(4) The pseudogroups $\mathcal{P}_{E}$ and $\mathcal{P}_{F}$ are spatially isomorphic.

(5) The graphs $E$ and $F$ are (continuously) orbit equivalent.

(6) The topological full groups $\llbracket \mathcal{G}_{E} \rrbracket$ and $\llbracket \mathcal{G}_{F} \rrbracket$ are isomorphic as abstract groups.

Remark 10.14. Statement (5) in Corollary 10.13 coincides with Li's notion of continuous orbit equivalence for the partial dynamical systems associated to the graphs, cf. [40].

Remark 10.15. We remark that in Corollary 10.13 statements (1), (2) and (3) are always equivalent, statements (4) and (5) are always equivalent and they are implied by (1), (2) and (3). Furthermore, if the graphs satisfy Condition $(L)$, then statements (1)-(5) are equivalent. Additionally, the equivalence of (1) and (2) has recently been shown in greater generality by Carlsen, Ruiz, Sims and Tomforde [15]. The same is true for (1) and (3) by recent work of Steinberg [65], even with weaker assumptions on the coefficient ring $R$.

\section{Embedding Theorems}

In this final section, we will show that several classes of groupoids embed into a certain fixed graph groupoid - namely the groupoid of the graph that consists of a single vertex and two edges. These classes include graph groupoids and AFgroupoids. We will also discuss the induced embeddings of the associated graph algebras and the topological full groups.

\subsection{Embedding graph groupoids}

Let $E_{2}$ denote the graph with a single vertex $v$, and two edges $a$ and $b$ :

$E_{2}$

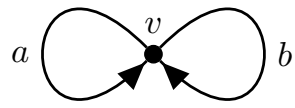

In 11, Brownlowe and Sørensen proved an algebraic analog of Kirchberg's Embedding Theorem (see 30]) for Leavitt path algebras. They showed that for any countable graph $E$, and for any commutative unital ring $R$, the Leavitt path algebra $L_{R}(E)$ embeds (unitally, whenever it makes sense) into $L_{R}\left(E_{2}\right)$. By inspecting their proof one finds that this embedding is also diagonal-preserving, i.e. that the canonical diagonal $D_{R}(E)$ is mapped into $D_{R}\left(E_{2}\right)$. A special case of Kirchberg's Embedding Theorem is that any graph $C^{*}$-algebra, $C^{*}(E)$, embeds into the Cuntz algebra $\mathcal{O}_{2}$, which is canonically isomorphic to the graph $C^{*}$-algebra $C^{*}\left(E_{2}\right)$ (and the groupoid $C^{*}$-algebra $C_{r}^{*}\left(\mathcal{G}_{E_{2}}\right)$ ). We denote the canonical diagonal subalgebra in $\mathcal{O}_{2}$ by $\mathcal{D}_{2}$. A priori, Kirchberg's embedding is of an analytic nature, but Brownlowe and Sørensen's results shows that in the case of graph $C^{*}$-algebras, algebraic embeddings exist. Both graph $C^{*}$-algebras and Leavitt path algebras have 
the same underlying groupoid models (being canonically isomorphic to the groupoid $C^{*}$-algebra, and the Steinberg $R$-algebra $\left(A_{R}\left(\mathcal{G}_{E}\right)\right)$ of $\mathcal{G}_{E}$, respectively). Generally, isomorphisms of the graph groupoids correspond to diagonal preserving isomorphisms of the algebras. Thus, one could wonder whether there is an embedding of the underlying graph groupoids. We will show that this is indeed the case, modulo topological obstructions. Our proof is inspired by [11, Proposition 5.1] (and the examples following it).

Lemma 11.1. Let $E$ be a countable graph with no sinks, no semi-tails, and suppose $E$ satisfies Condition $(L)$. Then there exists an injective local homeomorphism $\phi: \partial E \rightarrow E_{2}^{\infty}$ such that

$$
\phi \circ \llbracket \mathcal{G}_{E} \rrbracket \subseteq \llbracket \mathcal{G}_{E_{2}} \rrbracket \circ \phi .
$$

If $E^{0}$ is finite, then $\phi$ is surjective (hence a homeomorphism), and if $E^{0}$ is infinite, then $\phi(\partial E)=E_{2}^{\infty} \backslash\left\{a^{\infty}\right\}$. In particular, there exists an injective étale homomorphism

$$
\Phi: \operatorname{Germ}\left(\llbracket \mathcal{G}_{E} \rrbracket, \partial E\right) \rightarrow \mathcal{G}_{E_{2}}
$$

Proof. For transparency we first treat the case when $E^{0}$ is finite. The infinite case requires only a minor tweak. Let $n=\left|E^{0}\right|$. Label the vertices and edges of $E$ (arbitrarily) as

$$
E^{0}=\left\{w_{1}, w_{2}, \ldots, w_{n}\right\} \quad \text { and } \quad w_{i} E^{1}=\left\{e_{i, j} \mid 1 \leq j \leq k(i)\right\} \quad \text { for each } 1 \leq i \leq n,
$$

where $k(i)=\left|s^{-1}\left(w_{i}\right)\right|$. When $w_{i}$ is an infinite emitter, $k(i)=\infty$, and we let $j$ range over $\mathbb{N}$. For each pair $j, i$ with $j \in \mathbb{N}, i \in \mathbb{N} \cup\{\infty\}$ and $j \leq i$ we define a finite path $\alpha_{j, i} \in E_{2}^{*}$ as follows: $\alpha_{1,1}:=v$ and for $j \geq 2$

$$
\alpha_{j, i}:= \begin{cases}b & \text { if } j=1, \\ a^{j-1} b & \text { if } 1<j<i, \\ a^{j-1} & \text { if } j=i .\end{cases}
$$

Observe that for each fixed $i \in \mathbb{N}$, the set $\left\{Z\left(\alpha_{j, i}\right) \mid 1 \leq j \leq i\right\}$ forms a partition of $E_{2}^{\infty}$. And for $i=\infty,\left\{Z\left(\alpha_{j, i}\right) \mid 1 \leq j<\infty\right\}$ forms a partition of $E_{2}^{\infty} \backslash\left\{a^{\infty}\right\}$.

We now define the map $\phi: \partial E \rightarrow E_{2}^{\infty}$ as follows. For an infinite path $x=e_{i_{1}, j_{1}} e_{i_{2}, j_{2}} \ldots$ in $E$ we set

$$
\phi(x)=\alpha_{i_{1}, n} \alpha_{j_{1}, k\left(i_{1}\right)} \alpha_{j_{2}, k\left(i_{2}\right)} \cdots
$$

If $w_{i} \in E^{0}$ is an infinite emitter, then

$$
\phi\left(w_{i}\right)=\alpha_{i, n} a^{\infty} .
$$

For notational convenience, we define

$$
\phi^{*}(\mu):=\alpha_{i_{1}, n} \alpha_{j_{1}, k\left(i_{1}\right)} \alpha_{j_{2}, k\left(i_{2}\right)} \ldots \alpha_{j_{m}, k\left(i_{m}\right)} \in E_{2}^{*}
$$


for each finite path $\mu=e_{i_{1}, j_{1}} e_{i_{2}, j_{2}} \ldots e_{i_{m}, j_{m}} \in E^{*}$. Finally, if $\mu$ is a finite boundary path, then

$$
\phi(\mu)=\phi^{*}(\mu) a^{\infty} .
$$

Recall that $v \alpha=\alpha=\alpha v$ for each $\alpha \in E_{2}^{*}$. A priori, $\phi(x)$ could be a finite path in $E_{2}$. We argue that this is not the case. For a finite path $\mu \in E^{*}, \phi(\mu)$ is clearly infinite. For an infinite path $x=e_{i_{1}, j_{1}} e_{i_{2}, j_{2}} \ldots, \phi(x)$ is finite if and only if for some $M \in \mathbb{N}, \alpha_{j_{m}, k\left(i_{m}\right)}=v$ for all $m>M$, that is $k\left(i_{m}\right)=1$ and $j_{m}=1$. This means that $e_{i_{M+1}, j_{M+1}} e_{i_{M+2}, j_{M+2}} \ldots$ is either a semi-tail, or an eventually periodic point whose cycle has no exit. But there are by assumption no such paths in $E$. So we conclude that $\phi$ is well-defined.

Using the fact that $\left\{Z\left(\alpha_{j, i}\right)\right\}$ for fixed $i$ forms a partition of $E_{2}^{\infty}$, or $E_{2}^{\infty} \backslash\left\{a^{\infty}\right\}$, one easily sees that $\phi$ is a bijection. As for continuity, we define the finite subset $F_{i, l}:=\left\{e_{i, 1}, e_{i, 2}, \ldots, e_{i, l}\right\} \subseteq w_{i} E^{1}$ for $1 \leq l<k\left(w_{i}\right)+1$. Let $\mu=e_{i_{1}, j_{1}} e_{i_{2}, j_{2}} \ldots e_{i_{m}, j_{m}} \in E^{*}$ and suppose $r(\mu)=w_{i}$. Observe that

$$
\phi(Z(\mu))=Z\left(\phi^{*}(\mu)\right)
$$

and

$$
\phi\left(Z\left(\mu \backslash F_{i, l}\right)\right)=Z\left(\phi^{*}(\mu) a^{l}\right) .
$$

For arbitrary $F=\left\{e_{i, j_{1}}, \ldots, e_{i, j_{m}}\right\}$, we have

$$
Z(\mu \backslash F)=Z\left(\mu \backslash F_{i, j_{m}+1}\right) \sqcup \bigsqcup_{j \in J_{F}} Z\left(\mu e_{i, j}\right),
$$

where $J_{F}$ is the set of $j$ 's with $1 \leq j \leq j_{m}$ and $e_{i, j} \notin F$. Thus $\phi$ is an open map. Conversely, we have that for $\beta \in E_{2}^{*}$

$$
\phi^{-1}(Z(\beta))=\left(\bigcup_{\beta \leq \phi^{*}(\mu)} Z(\mu)\right) \cup\left(\bigcup_{l=1}^{\infty} \bigcup_{\beta \leq \phi^{*}(\lambda) a^{l}} Z\left(\lambda \backslash F_{r(\lambda), l}\right)\right),
$$

(and these unions may actually be taken to be finite). Hence $\phi$ is a homeomorphism.

To see that $\phi \circ \llbracket \mathcal{G}_{E} \rrbracket \circ \phi^{-1} \subseteq \llbracket \mathcal{G}_{E_{2}} \rrbracket$, let $\mu, \lambda \in E^{*}$ with $r(\mu)=r(\lambda)=w_{i}$ be given, and let $1 \leq l<k\left(w_{i}\right)+1$. Observe that

$$
\phi \circ \pi_{Z(\mu, \lambda)} \circ \phi^{-1}=\pi_{Z\left(\phi^{*}(\mu), \phi^{*}(\lambda)\right)}: Z\left(\phi^{*}(\lambda) \rightarrow Z\left(\phi^{*}(\mu)\right),\right.
$$

and

$$
\phi \circ \pi_{Z\left(\mu, F_{l}, \lambda\right)} \circ \phi^{-1}=\pi_{Z\left(\phi^{*}(\mu) a^{l}, \phi^{*}(\lambda) a^{l}\right)}: Z\left(\phi^{*}(\lambda) a^{l} \rightarrow Z\left(\phi^{*}(\mu) a^{l}\right),\right.
$$

as partial homeomorphisms. Utilizing a similar decomposition as in Eq. (11.1) for the basic set $Z(\mu, F, \lambda)$ for arbitrary $F$, together with the description of elements in $\llbracket \mathcal{G}_{E} \rrbracket$ from Proposition 9.4 we see that for each $\pi_{U} \in \llbracket \mathcal{G}_{E} \rrbracket$, the homeomorphism $\phi \circ \pi_{U} \circ \phi^{-1}$ belongs to $\llbracket \mathcal{G}_{E_{2}} \rrbracket$.

In the case that $E^{0}$ is infinite, all the arguments above still go through, with the minor adjustment that the first word in $\phi(x)$ is $\alpha_{i_{1}, \infty}$. This word always ends with $b$, so we see that $\phi$ becomes a homeomorphism from $\partial E$ onto $E_{2}^{\infty} \backslash\left\{a^{\infty}\right\}$. 
The final statement follows from Corollary 5.5 and Proposition $4.10\left(\llbracket \mathcal{G}_{E_{2}} \rrbracket\right.$ covers $\mathcal{G}_{E_{2}}$ since $\mathcal{G}_{E_{2}}$ is minimal).

Remark 11.2. The local homeomorphism $\phi$ constructed in the preceding proof depends on the choice of labeling of the graph. And there are of course many ways to label a graph, but each one gives a local homeomorphism $\phi$ with the desired properties.

In order to conclude that $\mathcal{G}_{E}$ embeds into $\mathcal{G}_{E_{2}}$ it seems like we have to assume that $\llbracket \mathcal{G}_{E} \rrbracket$ covers $\mathcal{G}_{E}$ (as this is not always the case). However, in the proof of Lemma 11.1, we are really showing that $\phi \circ \mathcal{P}_{c}\left(\mathcal{G}_{E}\right) \subseteq \mathcal{P}_{c}\left(\mathcal{G}_{E_{2}}\right) \circ \phi$, where $\mathcal{P}_{c}(\mathcal{G})$ denotes the inverse semigroup of partial homeomorphisms $\pi_{U}: s(U) \rightarrow r(U)$ coming from compact bisections $U \subseteq \mathcal{G}$. It is a sub-inverse semigroup of Renault's pseudogroup as in [10] 61] (when $\mathcal{G}$ is effective). The constructions in Secs. 4 and 5] apply more or less verbatim to $\mathcal{P}_{c}(\mathcal{G})$ as well. The crucial difference is that $\mathcal{P}_{c}(\mathcal{G})$ always covers $\mathcal{G}$, when $\mathcal{G}$ is ample. Thus, the analogs of Corollary 5.5] and Proposition 4.10 for $\mathcal{P}_{c}\left(\mathcal{G}_{E}\right)$ applied to $\phi$ induces the desired embedding of the graph groupoids - which we record in the following theorem.

Theorem 11.3. Let $E$ be a countable graph satisfying Condition $(L)$ and having no sinks nor semi-tails. Then there is an embedding of étale groupoids $\Phi: \mathcal{G}_{E} \hookrightarrow \mathcal{G}_{E_{2}}$. If $E^{0}$ is finite, then $\Phi$ maps $\partial E$ onto $E_{2}^{\infty}$.

Remark 11.4. Theorem 11.3 is optimal in the sense there is no embedding if one relaxes the assumptions on $E$. For if $\partial E$ has isolated points, then there is no local homeomorphism from $\partial E$ to $E_{2}^{\infty}$, as the latter has no isolated points. And if $E$ is uncountable, then there is no embedding either, for then $\partial E$ is not second countable, while $E_{2}^{\infty}$ is. Similarly, $\partial E$ cannot map onto $E_{2}^{\infty}$ if $E^{0}$ is infinite, for then the former is not compact.

\subsection{Diagonal embeddings of graph algebras}

From Theorem 11.3, we recover Brownlowe and Sørensen's embedding theorem for Leavitt path algebras (albeit for the slightly smaller class of graphs $E$ with $\partial E$ having no isolated points). However, we get the additional conclusion that when $E^{0}$ is finite (i.e. the algebras are unital), the embedding can be chosen to not only be unital, but also to map the diagonal onto the diagonal.

Corollary 11.5. Let $E$ be a countable graph with no sinks, no semi-tails, and satisfying Condition $(L)$.

(1) There is a diagonal preserving injective *-homomorphism $\psi: C^{*}(E) \rightarrow \mathcal{O}_{2}$, that is $\psi(\mathcal{D}(E)) \subseteq \mathcal{D}_{2}$. If $E^{0}$ is finite, then $\psi$ is unital and $\psi(\mathcal{D}(E))=\mathcal{D}_{2}$.

(2) For any commutative unital ring $R$, there is an injective *-algebra homomorphism $\rho: L_{R}(E) \rightarrow L_{R}\left(E_{2}\right)$ such that $\rho\left(D_{R}(E)\right) \subseteq D_{R}\left(E_{2}\right)$. If $E^{0}$ is finite, then $\rho$ is unital and $\rho\left(D_{R}(E)\right)=D_{R}\left(E_{2}\right)$. 
Remark 11.6. For each labeling of a graph $E$ as in the proof of Lemma 11.1 one obtains explicit embeddings of both the graph $C^{*}$-algebras and the Leavitt path algebras into $\mathcal{O}_{2}$ and $L_{R}\left(E_{2}\right)$, respectively, in terms of their canonical generators. This is done by expanding the scheme in [11, Proposition 5.1]. The canonical isomorphism between both $C^{*}(E)$ and $C^{*}\left(\mathcal{G}_{E}\right)$, and $L_{R}(E)$ and $A_{R}\left(\mathcal{G}_{E}\right)$ is given by $p_{v} \leftrightarrow 1_{Z(v)}$ for $v \in E^{0}$ (vertex projections) and $s_{e} \leftrightarrow 1_{Z(e, r(e))}$ for $e \in E^{1}$ (edge partial isometries). Denote the generators in $\mathcal{O}_{2}$ and $L_{R}\left(E_{2}\right)$ by $s_{a}$ and $s_{b}$. Given a labeling $E^{0}=\left\{w_{1}, w_{2}, w_{3}, \ldots\right\}$ and $E^{1}=\left\{e_{i, j} \mid 1 \leq i \leq n, 1 \leq j \leq k(i)\right\}$, the embedding of the algebras induced by $\phi$ as in Lemma 11.1 is given on the generators by

$$
p_{w_{i}} \mapsto s_{\phi^{*}\left(w_{i}\right)}\left(s_{\phi^{*}\left(w_{i}\right)}\right)^{*}, \quad s_{e_{i, j}} \mapsto s_{\phi^{*}\left(e_{i, j}\right)}\left(s_{\phi^{*}\left(r\left(e_{i, j}\right)\right)}\right)^{*},
$$

where $\phi^{*}(\mu) \in\{a, b\}^{*}$ is as in the proof of Lemma 11.1 (recall that for a finite path $\mu=e_{1}, \ldots, e_{n} \in E^{*}$, one defines $\left.s_{\mu}:=s_{e_{1}} \cdots s_{e_{2}}\right)$.

Remark 11.7. In the case that $E$ has infinitely many vertices, the image of the diagonals in Corollary 11.5 can be described as follows:

$$
\psi(\mathcal{D}(E))=\overline{\operatorname{span}\left\{s_{\alpha} s_{\alpha}^{*} \mid \alpha \in E_{2}^{*} \backslash\left\{a, a^{2}, a^{3}, \ldots\right\}\right\}}
$$

and

$$
\rho\left(D_{R}(E)\right)=\operatorname{span}_{R}\left\{s_{\alpha} s_{\alpha}^{*} \mid \alpha \in E_{2}^{*} \backslash\left\{a, a^{2}, a^{3}, \ldots\right\}\right\} .
$$

For examples of explicit embeddings for finite graphs satisfying Condition $(L)$ (possibly even having sinks), see [11 Sec. 5]. As for infinite graphs, we provide a few examples below.

Example 11.8. Consider the following graph, whose graph $C^{*}$-algebra is the Cuntz algebra $\mathcal{O}_{\infty}$ :

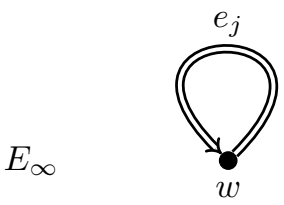

The double arrow indicates infinitely many edges, i.e. $E^{1}=\left\{e_{1}, e_{2}, e_{3}, \ldots\right\}$. For simplicity, we denote the edge isometries by $s_{j}$ for $j \in \mathbb{N}$. We use the labeling $w=w_{1}$ and $e_{j}=e_{1, j}$. Following the recipe in Remark 11.6, we obtain a unital embedding of $\mathcal{O}_{\infty}$ into $\mathcal{O}_{2}$ (and similarly of $L_{R}\left(E_{\infty}\right)$ into $L_{R}\left(E_{2}\right)$ ) which maps the diagonal onto the diagonal, in terms of generators as follows:

$$
p_{w}=1_{\mathcal{O}_{\infty}} \mapsto 1_{\mathcal{O}_{2}}=p_{v}, \quad s_{j} \mapsto s_{a^{j-1} b}
$$


Example 11.9. Next, consider the following graph:

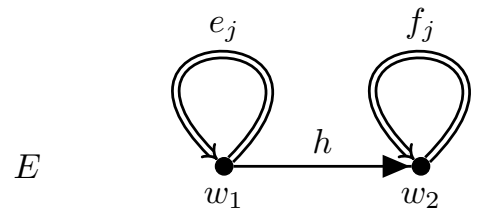

By labeling the edges as $h=e_{1,1}, e_{j}=e_{1, j+1}, f_{j}=e_{2, j}$ we get the following unital diagonal preserving embedding of $C^{*}(E)$ into $\mathcal{O}_{2}$ :

$$
\begin{aligned}
p_{w_{1}} & \mapsto s_{b} s_{b}^{*}, \quad p_{w_{2}} \mapsto s_{a} s_{a}^{*}, \\
s_{h} & \mapsto s_{b b} s_{a}^{*}, \quad s_{e_{j}} \mapsto s_{b a^{j} b} s_{b}^{*}, \quad s_{f_{j}} \mapsto s_{b a^{j} b} s_{a}^{*} .
\end{aligned}
$$

Example 11.10. Finally, let us look at a graph with infinitely many vertices:

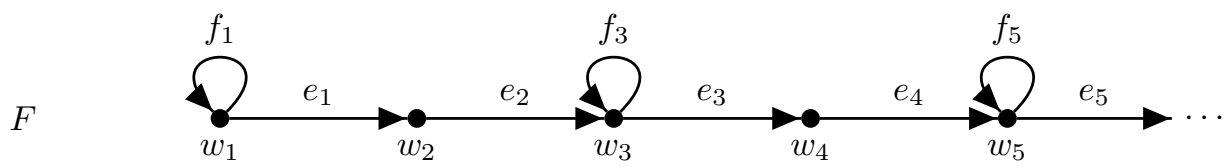

We label the edges as $e_{j}=e_{j, 1}$ for $j \in \mathbb{N}$, and $f_{j}=e_{j, 2}$ for $j$ odd. The induced diagonal preserving embedding of $C^{*}(F)$ into $\mathcal{O}_{2}$ is then given on the generators as follows:

$$
\begin{aligned}
& p_{w_{i}} \mapsto s_{a^{i-1} b}\left(s_{a^{i-1} b}\right)^{*}, s_{f_{j}} \mapsto s_{a^{j-1} b a}\left(s_{a^{j-1} b}\right)^{*} \quad(j \text { odd }), \\
& s_{e_{j}} \mapsto \begin{cases}s_{a^{j-1} b}\left(s_{a^{j} b}\right)^{*} & j \text { even, } \\
s_{a^{j-1} b^{2}}\left(s_{a^{j} b}\right)^{*} & j \text { odd. }\end{cases}
\end{aligned}
$$

\subsection{Analytic properties of $\llbracket \mathcal{G}_{E} \rrbracket$}

Before generalizing the groupoid embedding theorem to a larger class of groupoids in the next subsection we take brief pause to discuss some analytic properties of the topological full groups $\llbracket \mathcal{G}_{E} \rrbracket$ for graphs $E$ as in Lemma 11.1. First of all, $\llbracket \mathcal{G}_{E} \rrbracket$ is generally not amenable, as it often contains free products [49, Proposition 4.10].

Let $E_{n}$ for $n \geq 2$ denote the graph consisting of a single vertex and $n$ edges. And more generally, for $r \in \mathbb{N}$, let $E_{n, r}$ be the graph with $r$ vertices $w_{1}, w_{2}, \ldots, w_{r}$ and $n+r-1$ edges $e_{1}, \ldots, e_{n}, f_{1}, \ldots, f_{r-1}$ such that $s\left(e_{i}\right)=w_{1}, r\left(e_{i}\right)=w_{r}$ for each $1 \leq i \leq n$ and $s\left(f_{i}\right)=w_{i+1}, r\left(f_{i}\right)=w_{i}$ for each $1 \leq i \leq r-1$. According to 49 , Sec. 6], the topological full group $\llbracket \mathcal{G}_{E_{n, r}} \rrbracket$ is isomorphic to the Higman-Thompson group $V_{n, r}$. In particular, $\llbracket \mathcal{G}_{E_{2}} \rrbracket \cong V_{2,1}=V$ (Thompson's group $V$ ). As Lemma 11.1 in particular induces an algebraic embedding of the topological full groups, we have that $\llbracket \mathcal{G}_{E} \rrbracket$ embeds into $V$ for each graph $E$ as in Lemma 11.1 Thus, Lemma 11.1 may be considered a generalization of the well-known embedding of $V_{n, r}$ into $V$. As $V$ has the Haagerup property 24 , we deduce that $\llbracket \mathcal{G}_{E} \rrbracket$ does as well. 
Corollary 11.11. Let $E$ be a countable graph with no sinks, no semi-tails, and suppose $E$ satisfies Condition $(L)$. Then the topological full group $\llbracket \mathcal{G}_{E} \rrbracket$ has the Haagerup property.

Remark 11.12. For finite, strongly connected graphs, this was proved directly, using so-called zipper actions, by Matui in [49. Later, in [50], Matui proved that for any finite, strongly connected graph $E, \llbracket \mathcal{G}_{E} \rrbracket$ embeds into $\llbracket \mathcal{G}_{E_{2}} \rrbracket$. In fact, he proved even more, namely that $\mathcal{G}_{E_{2}}$ could be replaced by any groupoid with similar properties (see [50, Proposition 5.14] for the details). By our results, one may relax the conditions on $E$ considerably in Matui's embedding result.

\subsection{Embedding equivalent groupoids}

We are now going to expand on the embedding theorem for graph groupoids to include all groupoids that are merely groupoid equivalent to a graph groupoid. To accomplish this we will make us of the fundamental results by Carlsen, Ruiz and Sims in [14. Following their notation, let $\mathcal{R}$ denote the countably infinite discrete full equivalence relation, that is $\mathcal{R}=\mathbb{N} \times \mathbb{N}$ equipped with the discrete topology, whose product and inverse are given by $(k, m) \cdot(m, n):=(k, n)$ and $(m, n)^{-1}:=(n, m)$. We refer to the product groupoid $\mathcal{G} \times \mathcal{R}$ as the stabilization of the groupoid $\mathcal{G}$. For a graph $E$, let $S E$ denote the graph obtained from $E$ by adding a head at every vertex - see the example below (see also [68]). It is shown in [14] that $\mathcal{G}_{E} \times \mathcal{R} \cong \mathcal{G}_{S E}$ as topological groupoids for any graph $E$.

Example 11.13. The stabilized graph of $E_{2}$ is the following graph:

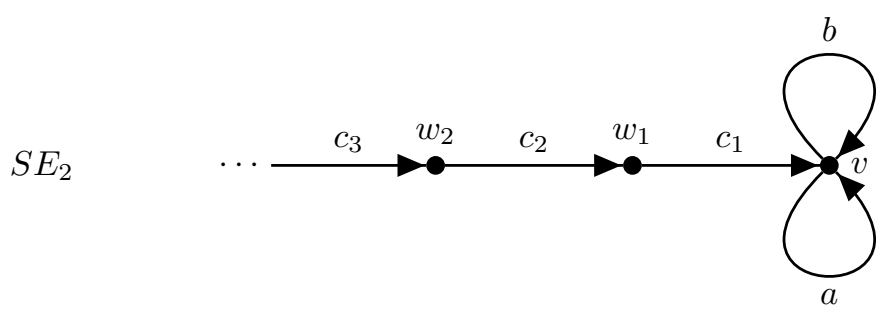

Let us first just say a few words on necessary conditions for an étale groupoid $\mathcal{H}$ to be embeddable into $\mathcal{G}_{E_{2}}$. First of all, it is clearly necessary that $\mathcal{H}$ is ample, Hausdorff and second countable, since $\mathcal{G}_{E_{2}}$ is. As we observed for the graph groupoids, it is also necessary that $\mathcal{H}^{(0)}$ has no isolated points, and hence that $\mathcal{H}^{(0)}$ is a locally compact Cantor space. Furthermore, since subgroupoids of effective groupoids are effective, it is also necessary that $\mathcal{H}$ be effective. As a final observation in this regard, any embedding $\Phi: \mathcal{H} \hookrightarrow \mathcal{G}_{E_{2}}$ induces an embedding of the isotropy bundles $\mathcal{H}^{\prime} \hookrightarrow\left(\mathcal{G}_{E_{2}}\right)^{\prime}$, meaning that $\Phi$ restricts to an embedding of the isotropy group $\mathcal{H}_{y}^{y}$ into $\left(\mathcal{G}_{E_{2}}\right)_{\Phi(y)}^{\Phi(y)}$ for each $y \in \mathcal{H}^{(0)}$. Now recall that for any graph 
groupoid $\mathcal{G}_{E}$ the isotropy groups are

$$
\left(\mathcal{G}_{E}\right)_{x}^{x} \cong \begin{cases}\mathbb{Z} & \text { if } x \text { is eventually periodic }, \\ 0 & \text { otherwise. }\end{cases}
$$

Thus, a final necessary condition for embeddability is that the isotropy bundle of $\mathcal{H}$ consists only of the trivial group and $\mathbb{Z}$. This rules out for instance (most) products of graph groupoids, since they typically have isotropy groups that are free abelian of rank up to the number of factors in the product. Note however, that taking the product with a principal groupoid does no harm in this regard. As we'll see imminently, taking the product with $\mathcal{R}$ (i.e. stabilizing) does not affect embeddability into $\mathcal{G}_{E_{2}}$.

Proposition 11.14. Let $\mathcal{H}$ be an effective ample second countable Hausdorff groupoid with $\mathcal{H}^{(0)}$ a locally compact Cantor space. Then $\mathcal{H}$ embeds into $\mathcal{G}_{E_{2}}$ if and only if the stabilized groupoid $\mathcal{H} \times \mathcal{R}$ embeds into $\mathcal{G}_{E_{2}}$.

Proof. The "if statement" is trivial as a groupoid always embeds into its stabilization. Suppose $\Phi: \mathcal{H} \rightarrow \mathcal{G}_{E_{2}}$ is an injective étale homomorphism. Then $\phi \times$ id $: \mathcal{H} \times \mathcal{R} \rightarrow \mathcal{G}_{E_{2}} \times \mathcal{R}$ is an injective étale homomorphism as well. By [14, Lemma 4.1] we have $\mathcal{G}_{E_{2}} \times \mathcal{R} \cong \mathcal{G}_{S E_{2}}$, and $S E_{2}$ is a countable graph satisfying Condition $(L)$ with no sinks nor semi-tails. So by Theorem $11.3 \mathcal{G}_{S E_{2}}$ embeds into $\mathcal{G}_{E_{2}}$. Thus $\mathcal{H} \times \mathcal{R}$ embeds into $\mathcal{G}_{E_{2}}$.

The next lemma shows that any étale embedding of a groupoid $\mathcal{H}$, with compact unit space, into $\mathcal{G}_{E_{2}}$ can be "twisted" into an embedding that hits the whole unit space of $\mathcal{G}_{E_{2}}$.

Lemma 11.15. Let $\mathcal{H}$ be an effective ample second countable Hausdorff groupoid with $\mathcal{H}^{(0)}$ a compact Cantor space. If $\mathcal{H}$ embeds into $\mathcal{G}_{E_{2}}$, then there exists an embedding $\Phi: \mathcal{H} \hookrightarrow \mathcal{G}_{E_{2}}$ such that $\Phi\left(\mathcal{H}^{(0)}\right)=E_{2}^{\infty}$.

Proof. Let $\Psi: \mathcal{H} \rightarrow \mathcal{G}_{E_{2}}$ be an injective étale homomorphism and let $Y=\Psi\left(\mathcal{H}^{(0)}\right)$. Then $Y$ is a compact open (hence clopen) subset of $E_{2}^{\infty}$. We claim that there exists a compact open bisection $U \subseteq \mathcal{G}_{E_{2}}$ such that $s(U)=Y$ and $r(U)=E_{2}^{\infty}$. The claim follows from [49, Theorem 6.4] and [51, Example 3.3(3)] by identifying $\mathcal{G}_{E_{2}}$ with the SFT-groupoid of the $1 \times 1$ matrix $A=[2]$ (see [51, Example 2.5]). Now define $\Phi(h)=U \cdot \Psi(h) \cdot U^{-1}$ for $h \in \mathcal{H}$. Then $\Phi$ is an injective étale homomorphism and

$$
\Phi\left(\mathcal{H}^{(0)}\right)=U Y U^{-1}=U U^{-1}=r(U)=E_{2}^{\infty} .
$$

We now state the most general version of our embedding theorem.

Theorem 11.16. Let $\mathcal{H}$ be an effective ample second countable Hausdorff groupoid whose unit space $\mathcal{H}^{(0)}$ is a locally compact Cantor space. If $\mathcal{H}$ is groupoid equivalent 
to $\mathcal{G}_{E}$, for some countable graph $E$ satisfying Condition $(L)$ and having no sinks nor semi-tails, then $\mathcal{H}$ embeds into $\mathcal{G}_{E_{2}}$. Moreover, if $\mathcal{H}^{(0)}$ is compact, then the embedding maps $\mathcal{H}^{(0)}$ onto $E_{2}^{\infty}$.

Proof. Suppose $\mathcal{H}$ is groupoid equivalent to $\mathcal{G}_{E}$ as above. Then by 14 Theorem 3.2] we have $\mathcal{H} \times \mathcal{R} \cong \mathcal{G}_{E} \times \mathcal{R}$. By Theorem 11.3 and Proposition 11.14 $\mathcal{G}_{E} \times \mathcal{R}$ embeds into $\mathcal{G}_{E_{2}}$, hence so does $\mathcal{H} \times \mathcal{R}$ and $\mathcal{H}$. The second statement follows from Lemma 11.15.

Remark 11.17. We note that for any groupoid $\mathcal{H}$ as in the above theorem, its topological full group $\llbracket \mathcal{H} \rrbracket$ also has the Haagerup property.

\subsection{Embedding AF-groupoids}

A well studied class of groupoids satisfying the hypothesis of Theorem [11.16] yet conceptually different from graph groupoids, are the AF-groupoids. See [28] (wherein they are dubbed $A F$-equivalence relations). Let $\mathcal{G}$ be an ample Hausdorff second countable groupoid with $\mathcal{G}^{(0)}$ a locally compact Cantor space. Then $\mathcal{G}$ is called an $A F$-groupoid if there exists an increasing sequence $\mathcal{K}_{1} \subseteq \mathcal{K}_{2} \subseteq \cdots \subseteq \mathcal{G}$ of clopen subgroupoids such that

- $\mathcal{K}_{n}$ is principal for each $n \in \mathbb{N}$.

- $\mathcal{K}_{n}^{(0)}=\mathcal{G}^{(0)}$ for each $n \in \mathbb{N}$.

- $\mathcal{K}_{n} \backslash \mathcal{G}^{(0)}$ is compact for each $n \in \mathbb{N}$.

- $\bigcup_{n=1}^{\infty} \mathcal{K}_{n}=\mathcal{G}$.

This entails that $\mathcal{G}$ is principal.

Remark 11.18. The terminology AF-groupoid is due to Renault [59], and is also used by Matui in [48, 51]. Note however, that Matui only considered the case of a compact unit space therein.

In the following example, we explain how Bratteli diagrams give rise to AFgroupoids.

Example 11.19 (cf. [28, Example 2.7(ii)]). A Bratteli diagram B is a directed graph whose vertex set $V$ and edge set $E$ can be written as countable disjoint unions of non-empty finite sets

$$
V=V_{0} \sqcup V_{1} \sqcup V_{2} \sqcup \cdots \quad \text { and } \quad E=E_{1} \sqcup E_{2} \sqcup E_{3} \sqcup \cdots
$$

such that the source and range maps satisfy $s\left(E_{n}\right)=V_{n-1}$ and $r\left(E_{n}\right) \subseteq V_{n}{ }^{\mathrm{i}}$ In particular, there are no sinks in $B$. Let $S_{B} \subseteq V$ denote the set of sources in $B$.

${ }^{\mathrm{i}}$ This notation is inconsistent with what we have been using for directed graphs so far. But since Bratteli diagrams are very special kinds of graphs we have chosen to use the well-established notation from the literature. In this way we can, albeit somewhat artificially, distinguish a Bratteli diagram from its underlying graph. 
Then $V_{0} \subseteq S_{B}$. We call $B$ a standard Bratteli diagram if there is only one source in $B$, i.e. $S_{B}=\left\{v_{0}\right\}=V_{0}$. We say that $B$ is simple if for every vertex $v \in V_{n}$, there is an $m>n$ such that there is a path from $v$ to every vertex in $V_{m}$. The partitions of the vertices and edges (into levels as in Eq. (11.2)) is considered part of the data of the Bratteli diagram $B$. We let $E_{B}$ denote the underlying graph where we "forget" about the partitions.

For a source $v \in S_{B} \cap V_{n}$ on level $n$ we let $X_{v}$ denote the set of infinite paths starting in $v$, that is

$X_{v}:=\left\{e_{n+1} e_{n+2} e_{n+3} \ldots \mid s\left(e_{n+1}\right)=v, e_{n+k} \in E_{n+k}, s\left(e_{n+k}\right)=r\left(e_{n+k-1}\right), k>1\right\}$.

The path space of $B$ is

$$
X_{B}:=\bigsqcup_{v \in S_{B}} X_{v}
$$

whose topology is given by the basis of cylinder sets

$$
C(\mu):=\left\{e_{n+1} e_{n+2} \ldots \in X_{s(\mu)} \mid e_{n+1} \ldots e_{n+|\mu|}=\mu\right\},
$$

where $\mu$ is a finite path such that $s(\mu)=v$ for some source $v \in S_{B} \cap V_{n}$. The path space $X_{B}$ is Boolean, and it is compact if and only if $S_{B}$ is finite. Further, $X_{B}$ is perfect if and only if $E_{B}$ has no semi-tails. Two infinite paths in $X_{B}$ are tail-equivalent if they agree from some level on. With this equivalence relation as the starting point, let for each $N \in \mathbb{N}$

$\mathcal{P}_{N}:=\left\{(x, y) \in X_{B} \times X_{B} \mid s(x) \in V_{m}, s(y) \in V_{n}, m, n \leq N, x_{k}=y_{k}\right.$ for all $\left.k>N\right\}$.

That is, $\mathcal{P}_{N}$ consists of all pairs of infinite paths which start before the $N$ th level and agrees from the $N$ th level and onwards. Equipping $\mathcal{P}_{N}$ with the relative topology from $X_{B} \times X_{B}$ makes $\mathcal{P}_{N}$ a compact principal ample Hausdorff groupoid whose unit space is identified with $\bigsqcup_{n=1}^{N} \bigsqcup_{v \in S_{B} \cap V_{n}} Z(v)$.

We define the groupoid of the Bratteli diagram $B$ as the increasing union

$$
\mathcal{G}_{B}:=\bigcup_{N=1}^{\infty} \mathcal{P}_{N}
$$

equipped with the inductive limit topology. For two finite paths $\mu, \lambda$ with $s(\mu), s(\lambda)$ in $S_{B}$ and $r(\mu)=r(\lambda)$ we define

$$
C(\mu, \lambda):=\left\{(x, y) \in C(\mu) \times C(\lambda) \mid x_{[|\mu|+1, \infty)}=y_{[|\lambda|+1, \infty)}\right\} .
$$

A straightforward computation shows that the family of $C(\mu, \lambda)$ 's form a compact open basis for the inductive limit topology on $\mathcal{G}_{B}$. We identify $\mathcal{G}_{B}^{(0)}$ with $X_{B}$. By setting $\mathcal{K}_{n}=\mathcal{P}_{n} \cup \mathcal{G}_{B}^{(0)}$ one sees that $\mathcal{G}_{B}$ is an AF-groupoid. The groupoid $\mathcal{G}_{B}$ is minimal if and only if $B$ is a simple Bratteli diagram.

Remark 11.20. Although the AF-groupoid $\mathcal{G}_{B}$ is defined in terms of a very special graph, namely the Bratteli diagram $B$, it is generally not isomorphic to a graph groupoid. To see this, recall that $\mathcal{G}_{B}$ is always principal, while a graph groupoid $\mathcal{G}_{E}$ 
is principal if and only if the graph $E$ has no cycles. If $X_{B}$ is compact, perfect and infinite (this is essentially stipulating that the Bratteli diagram is standard and "non-degenerate"), then $\mathcal{G}_{B}$ cannot be isomorphic to any graph groupoid. For any such $\mathcal{G}_{E}$ would have a compact unit space, i.e. $E$ has finitely many vertices, and $E$ would have no cycles and no sinks. But that is impossible.

Giordano, Putnam and Skau showed that, just as with AF-algebras [5], every AF-groupoid can be realized by a Bratteli diagram as in Example 11.19,

Theorem 11.21 ([28, Theorem 3.9]). Let $\mathcal{H}$ be an AF-groupoid. Then there exists a Bratteli diagram $B$ such that $H \cong \mathcal{G}_{B}$. If $\mathcal{H}^{(0)}$ is compact, then $B$ can be chosen to be standard.

Remark 11.22. As another example of a concrete description of the topological full group of an ample groupoid, we remark that Matui described the topological full group of an AF-groupoid with compact unit space in terms of a defining Bratteli diagram in [47, Proposition 3.3]. The topological full group $\llbracket \mathcal{G}_{B} \rrbracket$, where $B$ is a Bratteli diagram, is the direct limit of the finite groups $\Gamma_{N}$ for $N \in \mathbb{N}$, where $\Gamma_{N} \leq \operatorname{Homeo}\left(X_{B}\right)$ consists of all permutations of the finite set of paths from level $V_{0}$ to $V_{N}$ such that the permutation preserves the range of these paths (and the action on $X_{B}$ is by permuting the initial segment of an infinite path). We should also mention that these groups were originally studied by Krieger in [32, without emphasis on the underlying groupoids.

By the preceding remark it is clear that the topological full group of any AFgroupoid is a locally finite group. And actually, this characterizes the AF-groupoids. This is somewhat of a folklore result, but a proof is published by Matui in the compact case, and it is not hard to see that his proof extends to locally compact unit spaces as well.

Proposition 11.23 (cf. [47, Proposition 3.2]). Let $\mathcal{G}$ be an ample principal Hausdorff second countable groupoid with $\mathcal{G}^{(0)}$ a locally compact Cantor space. Then the topological full group $\llbracket \mathcal{G} \rrbracket$ is locally finite if and only if $\mathcal{G}$ is an AF-groupoid.

Remark 11.24. The commutator subgroups $\mathrm{D}(\mathcal{G}) \leq \llbracket \mathcal{G} \rrbracket$ for AF-groupoids $\mathcal{G}$ are quite interesting in their own right. In fact, these exhaust ${ }^{j}$ the class of so-called strongly diagonal limits of products of alternating groups (also called LDA-groups, see [37] where these are classified using the dimension groups of their Bratteli diagrams). These form a subclass of the locally finite simple groups. By Corollary 11.26 below, all the LDA-groups embed into Thompson's group $V$.

We now demonstrate that every AF-groupoid is groupoid equivalent to a graph groupoid. This is essentially just a reformulation of the main theorem from [21], wherein it is shown that any AF-algebra can be recovered as a certain pointed

${ }^{\mathrm{j}}$ With the single exception of the infinite finitary alternating group. 
graph $C^{*}$-algebra of a defining Bratteli diagram. In contrast, in Proposition 11.25 below we emphasize the groupoids, rather than their $C^{*}$-algebras. Also, since we use "unlabeled" Bratteli diagrams here, as opposed to labeled Bratteli diagrams (cf. [21, Sec. 2]), the computations are easier.

Proposition 11.25. Let $B$ be a Bratteli diagram. Then the $A F$-groupoid $\mathcal{G}_{B}$ is isomorphic to the restriction of the graph groupoid $\mathcal{G}_{E_{B}}$ to the open subset $\bigsqcup_{v \in S_{B}} Z(v)$ of $E_{B}^{\infty}$. In particular, every AF-groupoid is groupoid equivalent to a graph groupoid.

Proof. Let $A=\bigsqcup_{v \in S_{B}} Z(v)$. Then

$$
\left(\mathcal{G}_{E_{B}}\right)_{\mid A}=\left\{(x, k, y) \mid s(x), s(y) \in S_{B}, \sigma_{E_{B}}(x)^{m}=\sigma_{E_{B}}(y)^{n}, k=m-n\right\} .
$$

Due to the special structure of the graph $E_{B}$, the lag $k$ in $(x, k, y) \in\left(\mathcal{G}_{E_{B}}\right)_{\mid A}$ is uniquely determined by $x$ and $y$. In fact, $k$ is determined by the levels on which $x$ and $y$ start in the Bratteli diagram. Indeed, let $m, n \in \mathbb{N}$ be such that $s(x) \in V_{m}$ and $s(y) \in V_{n}$, then $k=n-m$. This means that the map $\Phi:\left(\mathcal{G}_{E_{B}}\right)_{\mid A} \rightarrow \mathcal{G}_{B}$ defined by $\Phi((x, k, y))=(x, y)$ is a bijection. It is easy to see that $\Phi$ is also a groupoid homomorphism. Finally, to see that $\Phi$ is a homeomorphism simply note that the family of $Z(\mu, \lambda)$ 's where $\mu, \lambda$ are finite paths with $s(\mu), s(\lambda) \in S_{B}$ and $r(\mu)=r(\lambda)$ form a basis for $\left(\mathcal{G}_{E_{B}}\right)_{\mid A}$, and that $\Phi(Z(\mu, \lambda))=C(\mu, \lambda)$. Thus $\left(\mathcal{G}_{E_{B}}\right)_{\mid A} \cong \mathcal{G}_{B}$ as étale groupoids.

We claim that $A$ is a $\mathcal{G}_{E_{B}}$-full subset of $E_{B}^{\infty}$, and then the second statement follows from [14, Theorem 3.2]. To see this, let $z \in E_{B}^{\infty}$ be an infinite path starting anywhere in the Bratteli diagram and simply note that by following $s(z)$ upwards in the Bratteli diagram, one eventually reaches a source $v \in S_{B}$ such that $v$ connects to $s(z)$. Letting $\mu$ be any path from $v$ to $s(z)$ we have that $z$ belongs to the $\mathcal{G}_{E_{B}}$-orbit of $\mu z \in A$.

As a special case of Theorem 11.16, we obtain the following corollary.

Corollary 11.26. Let $\mathcal{G}$ be an AF-groupoid with $\mathcal{G}^{(0)}$ perfect. Then there exists an embedding of étale groupoids $\mathcal{G} \hookrightarrow \mathcal{G}_{E_{2}}$. If $\mathcal{G}^{(0)}$ is compact, then $\mathcal{G}^{(0)}$ maps onto $E_{2}^{\infty}$.

From this we obtain an analogue of Corollary 11.5 for AF-algebras and their diagonals. Let $A$ be an AF-algebra. By an $A F$ Cartan subalgebra $D \subseteq A$ we mean a Cartan subalgebra arising from the diagonalization method of Strătilă and Voiculescu [67]. See [21, Sec. 4] for a description of these diagonals for non-unital AF-algebras. Note that they are also $C^{*}$-diagonals in the sense of Kumjian [34]. According to [61, Sec. 6.2] these are precisely the Cartain pairs arising as $\left(C_{r}^{*}\left(\mathcal{G}_{B}\right), C_{0}\left(X_{B}\right)\right)$ for a Bratteli diagram $B$.

Corollary 11.27. Let $A$ be an infinite-dimensional $A F$-algebra and let $D \subseteq A$ be any AF Cartan subalgebra in $A$ whose spectrum is perfect. Then there is an injective 
*-homomorphism $\psi: A \hookrightarrow \mathcal{O}_{2}$ such that $\psi(D) \subseteq \mathcal{D}_{2}$. If $A$ is unital, then so is $\psi$, and $\psi(D)=\mathcal{D}_{2}$.

Remark 11.28. As a final remark, we note that certain transformation groupoids (by virtue of actually being AF-groupoids) also embed into $\mathcal{G}_{E_{2}}$. Let $X$ be a noncompact locally compact Cantor space and let $T$ be a minimal homeomorphism on $X$. It follows from [28, Theorem 4.3] that the transformation groupoid $\mathbb{Z} \ltimes_{T} X$ is an AF-groupoid, and consequently $\mathbb{Z} \ltimes_{T} X$ embeds into $\mathcal{G}_{E_{2}}$.

An indirect way of seeing that $\mathbb{Z} \ltimes_{T} X$ is an AF-groupoid is via Proposition 11.23 By realizing the dynamical system $(X, T)$ as a so-called Bratteli-Vershik system on a (standard) almost simple ordered Bratteli diagram $B=(V, E, \geq)$ cf. [18, one easily observes (as Matui did in [46]) that $\llbracket \mathbb{Z} \ltimes_{T} X \rrbracket$ is locally finite. This is because each element of $\llbracket \mathbb{Z} \ltimes_{T} X \rrbracket$ only depends on the initial edges down to level $N$ for some fixed $N$ (determined by the group element), for each infinite path in $X_{B}$. This actually allows one to describe the topological full group $\llbracket \mathbb{Z} \ltimes_{T} X \rrbracket$ explicitly in terms of a conjugate Bratteli-Vershik system.

A third way of demonstrating that $\mathbb{Z} \ltimes_{T} X$ is an AF-groupoid is that one can go from a conjugate Bratteli-Vershik system on an ordered Bratteli dia$\operatorname{gram} B=(V, E, \geq)$ to an "unordered" Bratteli diagram $B^{\prime}$ such that $\mathbb{Z} \ltimes_{T} X \cong \mathcal{G}_{B^{\prime}}$ as étale groupoids. Indeed, let $e_{1} e_{2} e_{3} \ldots \in X_{B}$ denote the unique maximal and minimal path in $X_{B}$ (cf. [18]). By "forgetting" the ordering and removing each of the edges $e_{n}$ for all $n \in \mathbb{N}$, and thereby introducing a source at each of the vertices $s\left(e_{n}\right)$, one obtains the modified Bratteli diagram $B^{\prime}$, and it is not hard to see that the AF-groupoid $\mathcal{G}_{B^{\prime}}$ is isomorphic to $\mathbb{Z} \ltimes_{T} X$.

\section{Acknowledgments}

We would like to express our gratitude to Volodymyr Nekrashevych for sharing his private notes on Rubin's theorems. We would also like to thank Hiroki Matui for pointing out Lemma 4.3 to us, as well as for other valuable comments while he visited NTNU in the spring of 2018. The first named author thanks Eric Wofsey for helpful remarks on Stone duality. We also wish to thank Ulrik Enstad and Christian Skau for comments on the first draft of this paper. Further, we are grateful to Fredrik Hildrum for assistance with typesetting. Finally, we want to thank the anonymous referee for his or her thorough feedback.

\section{References}

[1] G. Abrams, P. Ara and M. Siles Molina, Leavitt Path Algebras, Lecture Notes in Mathematics, Vol. 2191 (Springer, London, 2017), xiii+287 pp.

[2] T. Bates, D. Pask, I. Raeburn and W. Szymański, The $C^{*}$-algebras of row-finite graphs, New York J. Math. 6 (2000) 307-324.

[3] S. Bezuglyi and K. Medynets, Full groups, flip conjugacy, and orbit equivalence of Cantor minimal systems, Colloq. Math. 110(2) (2008) 409-429.

[4] C. Bleak and D. Lanoue, A family of non-isomorphism results, Geom. Dedicata 146 (2010) 21-26. 
[5] O. Bratteli, Inductive limits of finite dimensional $C^{*}$-algebras, Trans. Amer. Math. Soc. 171 (1972) 195-234.

[6] M. G. Brin, Higher dimensional Thompson groups, Geom. Dedicata 108 (2004) 163192.

[7] K. A. Brix and E. Scarparo, $C^{*}$-simplicity and representations of topological full groups of groupoids, preprint (2018), arXiv:1805.06743v1.

[8] J. Brown, L. O. Clark, C. Farthing and A. Sims, Simplicity of algebras associated to étale groupoids, Semigroup Forum 88(2) (2014) 433-452.

[9] J. H. Brown, G. Nagy, S. Reznikoff, A. Sims and D. P. Williams, Cartan subalgebras in $C^{*}$-algebras of Hausdorff étale groupoids, Integral Equations Operator Theory 85(1) (2016) 109-126.

[10] N. Brownlowe, T. M. Carlsen and M. F. Whittaker, Graph algebras and orbit equivalence, Ergodic Theory Dynam. Syst. 37(2) (2017) 389-417.

[11] N. Brownlowe and A. P. W. Sørensen, Leavitt $R$-algebras over countable graphs embed into $L_{2, R}$, J. Algebra 454 (2016) 334-356.

[12] T. M. Carlsen, S. Eilers, E. Ortega and G. Restorff, Flow equivalence and orbit equivalence for shifts of finite type and isomorphism of their groupoids, J. Math. Anal. Appl. 469(2) (2019) 1088-1110.

[13] T. M. Carlsen and J. Rout, Diagonal-preserving graded isomorphisms of Steinberg algebras, Commun. Contemp. Math. 20(6) (2018), 1750064.

[14] T. M. Carlsen, E. Ruiz and A. Sims, Equivalence and stable isomorphism of groupoids, and diagonal-preserving stable isomorphisms of graph $C^{*}$-algebras and Leavitt path algebras, Proc. Amer. Math. Soc. 145(4) (2017) 1581-1592.

[15] T. M. Carlsen, E. Ruiz, A. Sims and M. Tomforde, Reconstruction of groupoids and $C^{*}$-rigidity of dynamical systems, preprint (2017), arXiv:1711.01052v1.

[16] T. M. Carlsen and M. L. Winger, Orbit equivalence of graphs and isomorphism of graph groupoids, Math. Scand. (2018) 123(2) 239-248.

[17] J. Cuntz and W. Krieger, A class of $C^{*}$-algebras and topological Markov chains, Invent. Math. 56(3) (1980) 251-268.

[18] A. I. Danilenko, Strong orbit equivalence of locally compact Cantor minimal systems, Internat. J. Math. 12(1) (2001) 113-123.

[19] V. Deaconu, Groupoids associated with endomorphisms, Trans. Amer. Math. Soc. 347(5) (1995) 1779-1786.

[20] H. P. Doctor, The categories of Boolean lattices, Boolean rings and Boolean spaces, Canad. Math. Bull. 7 (1964) 245-252.

[21] D. Drinen, Viewing AF-algebras as graph algebras, Proc. Amer. Math. Soc. 128(7) (2000) 1991-2000.

[22] D. Drinen and M. Tomforde, The $C^{*}$-algebras of arbitrary graphs, Rocky Mountain J. Math. 35(1) (2005) 105-135.

[23] H. A. Dye, On groups of measure preserving transformations. II, Amer. J. Math. 85 (1963) 551-576.

[24] D. S. Farley, Proper isometric actions of Thompson's groups on Hilbert space, Int. Math. Res. Not. 45 (2003) 2409-2414.

[25] D. H. Fremlin, Measure Theory, Measure Algebras, Vol. 3 (Torres Fremlin, Colchester, 2004), Corrected second printing of the 2002 original.

[26] T. Giordano, I. F. Putnam and C. F. Skau, Topological orbit equivalence and $C^{*}$ crossed products, J. Reine Angew. Math. 469 (1995) 51-111.

[27] T. Giordano, I. F. Putnam and C. F. Skau, Full groups of Cantor minimal systems, Israel J. Math. 111 (1999) 285-320. 
[28] T. Giordano, I. F. Putnam and C. F. Skau, Affable equivalence relations and orbit structure of Cantor dynamical systems, Ergodic Theory Dynam. Syst. 24(2) (2004) 441-475.

[29] K. Juschenko and N. Monod, Cantor systems, piecewise translations and simple amenable groups, Ann. of Math. (2) 178(2) (2013) 775-787.

[30] E. Kirchberg and N. C. Phillips, Embedding of exact $C^{*}$-algebras in the Cuntz algebra $\mathcal{O}_{2}$, J. Reine Angew. Math. 525 (2000) 17-53.

[31] S. Koppelberg, Handbook of Boolean Algebras, J. Donald Monk and R. Bonnet, eds., Vol. 1 (North-Holland Publishing Co., Amsterdam, 1989).

[32] W. Krieger, On a dimension for a class of homeomorphism groups, Math. Ann. 252(2) (1980) 87-95.

[33] G. Kudryavtseva and M. V. Lawson, Boolean sets, skew Boolean algebras and a non-commutative Stone duality, Algebra Universalis 75(1) (2016) 1-19.

[34] A. Kumjian, On $C^{*}$-diagonals, Canad. J. Math. 38(4) (1986) 969-1008.

[35] A. Kumjian, D. Pask and I. Raeburn, Cuntz-Krieger algebras of directed graphs, Pacific J. Math. 184(1) (1998) 161-174.

[36] A. Kumjian, D. Pask, I. Raeburn and J. Renault, Graphs, groupoids, and CuntzKrieger algebras, J. Funct. Anal. 144(2) (1997) 505-541.

[37] Y. Lavrenyuk and V. Nekrashevych, On classification of inductive limits of direct products of alternating groups, J. Lond. Math. Soc. (2) 75(1) (2007) 146-162.

[38] M. V. Lawson, A noncommutative generalization of Stone duality, J. Aust. Math. Soc. 88(3) (2010) 385-404.

[39] M. V. Lawson, Tarski monoids: Matui's spatial realization theorem, Semigroup Forum 95(2) (2017) 379-404.

[40] X. Li, Partial transformation groupoids attached to graphs and semigroups, Int. Math. Res. Not. IMRN 17 (2017) 5233-5259.

[41] X. Li, Continuous orbit equivalence rigidity, Ergodic Theory Dynam. Syst. 38(4) (2018) 1543-1563.

[42] K. Matsumoto, Orbit equivalence of topological Markov shifts and Cuntz-Krieger algebras, Pacific J. Math. 246(1) (2010) 199-225.

[43] K. Matsumoto, Full groups of one-sided topological Markov shifts, Israel J. Math. 205(1) (2015) 1-33.

[44] K. Matsumoto and H. Matui, Continuous orbit equivalence of topological Markov shifts and Cuntz-Krieger algebras, Kyoto J. Math. 54(4) (2014) 863-877.

[45] N. Matte Bon, Rigidity of graphs of germs and homomorphisms between full groups, preprint (2018), arXiv:1801.10133v1.

[46] H. Matui, Topological orbit equivalence of locally compact Cantor minimal systems, Ergodic Theory Dynam. Syst. 22(6) (2002) 1871-1903.

[47] H. Matui, Some remarks on topological full groups of Cantor minimal systems, Internat. J. Math. 17(2) (2006) 231-251.

[48] H. Matui, Homology and topological full groups of étale groupoids on totally disconnected spaces, Proc. Lond. Math. Soc. (3) 104(1) (2012) 27-56.

[49] H. Matui, Topological full groups of one-sided shifts of finite type, J. Reine Angew. Math. 705 (2015) 35-84.

[50] H. Matui, Étale groupoids arising from products of shifts of finite type, Adv. Math. 303 (2016) 502-548.

[51] H. Matui, Topological full groups of Étale groupoids, in Operator algebras and applications - The Abel Symposium 2015, Abel. Symp., Vol. 12 (2019) pp. 203-230.

[52] K. Medynets, Reconstruction of orbits of Cantor systems from full groups, Bull. Lond. Math. Soc. 43(6) (2011) 1104-1110. 
[53] F. J. Murray and J. von Neumann, On rings of operators. IV, Ann. of Math. (2) 44 (1943) 716-808.

[54] V. V. Nekrashevych, Simple groups of dynamical origin, Ergodic Theory Dynam. Syst., 39(3) (2019), 707-732.

[55] E. Ortega, Homology of the Katsura-Exel-Pardo groupoid, preprint (2018), arXiv:1806.09297v1.

[56] A. L. T. Paterson, Groupoids, Inverse Semigroups and Their Operator Algebras, Progress in Mathematics, Vol. 170 (Birkhäuser Boston, Inc., Boston, MA, 1999).

[57] N. C. Phillips, Crossed products of the Cantor set by free minimal actions of $\mathbb{Z}^{d}$, Comm. Math. Phys. 256(1) (2005) 1-42.

[58] I. Raeburn, Graph algebras, in CBMS Regional Conf. Series in Mathematics, published for the Conference Board of the Mathematical Sciences, Vol. 103 (Washington, DC; by the American Mathematical Society, Providence, RI, 2005).

[59] J. Renault, A Groupoid Approach to $C^{*}$-Algebras, Lecture Notes in Mathematics, Vol. 793 (Springer, Berlin, 1980).

[60] J. Renault, Cuntz-like algebras, in Operator Theoretical Methods (Timişoara, 1998) (Theta Found., Bucharest, 2000), pp. 371-386.

[61] J. Renault, Cartan subalgebras in $C^{*}$-algebras, Irish Math. Soc. Bull. 61 (2008) $29-63$.

[62] M. Rubin, On the reconstruction of topological spaces from their groups of homeomorphisms, Trans. Amer. Math. Soc. 312(2) (1989) 487-538.

[63] M. Rubin, Locally moving groups and reconstruction problems, in Ordered Groups and Infinite Permutation Groups, Mathematics Application, Vol. 354 (Kluwer Acad. Publ., Dordrecht, 1996), pp. 121-157.

[64] A. Sims, Étale groupoids and their $C^{*}$-algebras, preprint (2017), arXiv:1710.10897v1.

[65] B. Steinberg, Diagonal-preserving isomorphisms of étale groupoid algebras, J. Algebra 518 (2019) 412-439.

[66] M. H. Stone, Applications of the theory of Boolean rings to general topology, Trans. Amer. Math. Soc. 41(3) (1937) 375-481.

[67] Ş. Strătilă and D. Voiculescu, Representations of AF-Algebras and of the Group $U(\infty)$, Lecture Notes in Mathematics, Vol. 486 (Springer-Verlag, Berlin, New York, $1975)$.

[68] M. Tomforde, Stability of $C^{*}$-algebras associated to graphs, Proc. Amer. Math. Soc. 132(6) (2004) 1787-1795.

[69] S. B. G. Webster, The path space of a directed graph, Proc. Amer. Math. Soc. 142(1) (2014) 213-225. 